\title{
PARTIALLY HYPERBOLIC DIFFEOMORPHISMS AND LAGRANGIAN CONTACT STRUCTURES
}

\author{
MARTIN MION-MOUTON
}

\begin{abstract}
In this paper, we classify the three-dimensional partially hyperbolic diffeomorphisms whose stable, unstable, and central distributions $E^{s}, E^{u}$, and $E^{c}$ are smooth, such that $E^{s} \oplus E^{u}$ is a contact distribution, and whose non-wandering set equals the whole manifold. We prove that up to a finite quotient or a finite power, they are smoothly conjugated either to the time-one map of an algebraic contactAnosov flow, or to an affine partially hyperbolic automorphism of a nil-manifold. The rigid geometric structure induced by the invariant distributions plays a fundamental role in the proof.
\end{abstract}

\section{INTRODUCTION}

In a lot of natural situations, a differentiable dynamical system on a smooth manifold preserves a geometric structure on the tangent bundle, defined by invariant distributions. For instance, if it preserves a Borel measure, then Oseledet's theorem provides an almosteverywhere defined splitting of the tangent bundle, given by the rates of expansion or contraction of the tangent vectors by the differentials of the dynamics.

Although invariant geometric structures naturally arise, they are in general highly non-regular (Oseledet's decomposition is for instance only measurable), and this lack of regularity allows a lot of flexibility of the dynamics: former examples can be deformed in order to produce a lot of new ones. In contrast, the smoothness of the invariant distributions puts a strong restriction on the system, and the known examples with smooth (i.e. $\mathcal{C}^{\infty}$ ) distributions are in general "very symmetric": typically, they arise from compact quotient of Lie groups, with action by affine automorphisms.

It is thus natural to ask to what extent the geometric structure preserved by the dynamics makes the situation rigid, and especially why.

Let us give a paradigmatic example of rigidity with the following result of Étienne Ghys concerning three-dimensional Anosov flows (the statement proved by Ghys in [Ghy87] is more precise than the one given below).

Theorem 1.1 ([Ghy87]). Let $\left(\varphi^{t}\right)$ be an Anosov flow of a three-dimensional closed connected manifold. If the stable and unstable distributions of $\left(\varphi^{t}\right)$ are smooth, then:

- either $\left(\varphi^{t}\right)$ is smoothly conjugated to the suspension flow of a hyperbolic automorphism of the two-torus,

- or $\left(\varphi^{t}\right)$ is smoothly orbit equivalent to a finite covering of the geodesic flow of a compact hyperbolic surface.

Date: February 24, 2020 (last revision: January 11, 2021). 
We recall that a smooth non-singular flow $\left(\varphi^{t}\right)$ of a compact manifold $M$ is Anosov if its differentials preserve two distributions $E^{s}$ and $E^{u}$, respectively called the stable and unstable distribution of $\left(\varphi^{t}\right)$, satisfying $\mathrm{T} M=E^{s} \oplus \mathbb{R} \frac{d \varphi^{t}}{d t} \oplus E^{u}$ and such that $E^{s}$ is uniformly contracted by $\left(\varphi^{t}\right)$, and $E^{u}$ uniformly expanded by $\left(\varphi^{t}\right)$.

Under the smoothness assumption of $E^{s}$ and $E^{u}$, Ghys notices that the plane distribution $E^{s} \oplus E^{u}$ can only have two extreme geometrical behaviours: either it integrates into a foliation, or it is a contact distribution (i.e. it is locally the kernel of a contact oneform). In the first case, former results of Plante and Franks conclude the proof, and lead to the suspension examples. The work of Ghys in [Ghy87] is therefore almost entirely devoted to three-dimensional contact-Anosov flows, i.e. when $E^{s}$ and $E^{u}$ are smooth, and $E^{s} \oplus E^{u}$ is contact. Under these geometrical assumptions, the pair $\left(E^{s}, E^{u}\right)$ is a rigid geometric structure preserved by the Anosov flow, which makes the classification possible and leads to the finite coverings of geodesic flows.

In this paper, we investigate the same kind of geometrical rigidity conditions, but for the discrete-time analogs of Anosov flows that are the partially hyperbolic diffeomorphisms.

1.1. Principal results. We refer to [CP15] for a very complete introduction to partially hyperbolic diffeomorphisms, for which we use the following definition.

Definition 1.2. A smooth diffeomorphism $f$ of a compact manifold $M$ is partially hyperbolic if it preserves a splitting $\mathrm{T} M=E^{s} \oplus E^{u} \oplus E^{c}$ of the tangent bundle into three non-zero continuous distributions, satisfying the following dynamical conditions with respect to some Riemannian metric on $M$.

- The stable distribution $E^{s}$ is uniformly contracted by $f$, i.e. there is a non-zero integer $N$ such that for any $x \in M$ and any unit vector $v^{s} \in E^{s}(x)$,

$$
\left\|\mathrm{D}_{x} f^{N}\left(v^{s}\right)\right\|<1
$$

- The unstable distribution $E^{u}$ is uniformly expanded by $f$, i.e. uniformly contracted by $f^{-1}$.

- The splitting is dominated, i.e. there is a non-zero integer $N$ such that for any $x \in M$, and any unit vectors $v^{s} \in E^{s}(x), v^{c} \in E^{c}(x)$, and $v^{u} \in E^{u}(x)$,

$$
\left\|\mathrm{D}_{x} f^{N}\left(v^{s}\right)\right\|<\left\|\mathrm{D}_{x} f^{N}\left(v^{c}\right)\right\|<\left\|\mathrm{D}_{x} f^{N}\left(v^{u}\right)\right\| .
$$

$E^{c}$ is called the central distribution.

The three invariant distributions of a partially hyperbolic diffeomorphism have in general no reasons to be differentiable, but we study in this paper the particular case when they are smooth, i.e. $\mathcal{C}^{\infty}$, and when $E^{s} \oplus E^{u}$ is furthermore a contact distribution.

The (non-zero) time maps of the contact-Anosov flows appearing in Ghys Theorem 1.1 give us the first examples satisfying these geometrical conditions. They have the following nice algebraic description (see [Ghy87] for more details). Let us denote by $\tilde{A}=$ $\left\{a^{t}\right\}_{t \in \mathbb{R}}$ the one-parameter subgroup of the universal cover $\widetilde{\mathrm{SL}}_{2}(\mathbb{R})$ of $\mathrm{SL}_{2}(\mathbb{R})$ generated by $\left(\begin{array}{cc}1 & 0 \\ 0 & -1\end{array}\right) \in \mathfrak{s l}_{2}$. Then for any cocompact lattice $\Gamma_{0}$ of $\widetilde{S L}_{2}(\mathbb{R})$, the flow $\left(R_{a^{t}}\right)$ of right translations by $\tilde{A}$ on the quotient $\Gamma_{0} \backslash \widetilde{\mathrm{SL}}_{2}(\mathbb{R})$ is a finite covering of the geodesic flow 
of a compact hyperbolic surface (up to a constant rescaling of the time by a factor $\frac{1}{2}$ ), and is thus Anosov. Moreover, if a morphism $u: \Gamma_{0} \rightarrow \tilde{A}$ is such that the graph-group $\Gamma=\left\{(\gamma, u(\gamma)) \mid \gamma \in \Gamma_{0}\right\}$ acts freely, properly and cocompactly on $\widetilde{\mathrm{SL}}_{2}(\mathbb{R})$ by the action $(g, a) \cdot x=g x a$, then $\left(R_{a^{t}}\right)$ still induces an Anosov flow of the quotient $\Gamma \backslash \widetilde{S L}_{2}(\mathbb{R})$, which is a time-change of the former one (non-trivial if $u \neq \mathrm{id}$ ). We will call these flows the three-dimensional algebraic contact-Anosov flows.

In contrast, the following algebraic examples are the time-map of none Anosov flow. For $(\lambda, \mu) \in \mathbb{R}^{* 2}$, we consider the automorphism

$$
\varphi_{\lambda, \mu}:\left(\begin{array}{ccc}
1 & x & z \\
0 & 1 & y \\
0 & 0 & 1
\end{array}\right) \in \operatorname{Heis}(3) \mapsto\left(\begin{array}{ccc}
1 & \lambda x & \lambda \mu z \\
0 & 1 & \mu y \\
0 & 0 & 1
\end{array}\right) \in \operatorname{Heis}(3)
$$

of the Heisenberg group. If $\varphi=\varphi_{\lambda, \mu}, g \in \operatorname{Heis}(3), \Gamma$ is a cocompact lattice of Heis(3), and $g \varphi(\Gamma) g^{-1}=\Gamma$, then $L_{g} \circ \varphi(\Gamma x)=\Gamma(g \varphi(x))$ is a well-defined diffeomorphism of the nil-Heis(3)-manifold $\Gamma \backslash \operatorname{Heis}(3)$. If we moreover assume that either $|\lambda|<1$ and $|\mu|>1$, or the opposite, then $L_{g} \circ \varphi$ is a partially hyperbolic diffeomorphism, whose invariant distributions are smooth, and such that $E^{s} \oplus E^{u}$ is contact (see Paragraph 4.1.2). Concrete examples of cocompact lattices preserved by such automorphisms indeed exist, and we will call $L_{g} \circ \varphi$ a partially hyperbolic affine automorphism.

The principal result of this paper is that, assuming all points are non-wandering, there are no other examples than the two families we described precedently.

Theorem A. Let $M$ be a closed, connected and orientable three-dimensional manifold, and $f$ be a partially hyperbolic diffeomorphism of $M$ such that

- the stable, unstable, and central distributions $E^{s}, E^{u}$ and $E^{c}$ of $f$ are smooth,

- $E^{s} \oplus E^{u}$ is a contact distribution,

- and the non-wandering set $N W(f)$ equals $M$.

Then we have the following description.

(1) Either some finite power of $f$ is smoothly conjugated to a non-zero time-map of a three-dimensional algebraic contact-Anosov flow,

(2) or $f$ lifts by a smooth covering of order at most 4 to a partially hyperbolic affine automorphism of a nil-Heis(3)-manifold.

Actually, our geometrical conditions are so rigid that the uniformity of the contraction and the expansion of the diffeomorphism will be obtained as a byproduct.

Definition 1.3. We will say that a distribution $E$ of a compact manifold $M$ is weakly contracted by a diffeomorphism $f$, if for some Riemannian metric on $M$, we have for any $x \in M$ :

$$
\lim _{n \rightarrow+\infty}\left\|\left.\mathrm{D}_{x} f^{n}\right|_{E}\right\|=0 \text { or } \lim _{n \rightarrow-\infty}\left\|\left.\mathrm{D}_{x} f^{n}\right|_{E}\right\|=0 .
$$

We emphasize that the "direction" of weak contraction can a priori change from point to point, and that this notion is unchanged when replacing $f$ by $f^{-1}$.

Theorem B. Let $M$ be a closed, connected and orientable three-dimensional manifold, endowed with a smooth splitting $\mathrm{T} M=E^{\alpha} \oplus E^{\beta} \oplus E^{c}$ such that $E^{\alpha} \oplus E^{\beta}$ is a contact 
distribution. Let $f$ be a smooth diffeomorphism of $M$ that preserves this splitting, and such that

- each of the distributions $E^{\alpha}$ and $E^{\beta}$ is weakly contracted by $f$,

- and $f$ has a dense orbit.

Then the conclusions of Theorem A hold. In particular, $f$ is a partially hyperbolic diffeomorphism.

Theorem A will directly follow from Theorem B by an argument of Brin, as explained in Paragraph 8.2 at the end of this paper. We also give in this paragraph the precise satement of Theorem A, that does not use any domination hypothesis on $E^{c}$ (see Corollary 8.2). The rest of the paper is devoted to the proof of Theorem B.

The classification question for partially hyperbolic diffeomorphisms in dimension three has led to a lot of works in the recent years, and significant progress has been made concerning the general case, as can be seen for instance in the survey [HP18]. Recently, different additional rigidity conditions have also been studied.

Carrasco, Pujals and Rodriguez-Hertz obtain in [CPRH19] a classification result under the smoothness assumption of invariant distributions. On the contrary of Theorem A, no additional geometrical condition is assumed, but the authors assume that the differential of the partially hyperbolic diffeomorphism is constant when read in the global frame given by three smooth vector fields generating these distributions. The geometric structure $\left(E^{s}, E^{u}, E^{c}\right)$ defined by such a partially hyperbolic diffeomorphism is in general not rigid, and their result is obtained through dynamical arguments.

Beside the smoothness assumption on invariant distributions, Bonatti and Zhang obtain in [BZ19] different rigidity results in the continuous category, under specific dynamical assumptions.

1.2. A rigid geometric structure preserved by partially hyperbolic diffeomorphisms. Roughly speaking, a rigid geometric structure is a structure with "few automorphisms". More precisely, they are those smooth geometric structures whose Lie algebra of local Killing fields (i.e. local vector fields whose flow preserves the structure) is everywhere finite-dimensional.

As d'Ambra and Gromov pointed out in [GD91], it is natural to believe that rigid geometric structures preserved by rich dynamical systems have to be particularly peculiar: "one does not expect rigid geometry to be accompanied by rich dynamics" ([GD91, $\S 0.3$ p.21]). It seems thus reasonable to look for classification results in these situations. The general idea is that rich dynamical properties will imply strong restrictions on the rigid geometric structure, inducing in return a rigidity of the dynamical system itself.

Several rigid geometric structures can be preserved by a contact-Anosov flow $\left(\varphi^{t}\right)$. First of all, $\left(\varphi^{t}\right)$ always preserves a contact one-form $\alpha$ defined by $\alpha\left(\frac{d \varphi^{t}}{d t}\right)=1$ and $\left.\alpha\right|_{E^{s} \oplus E^{u}}=0$. If the dimension is $2 n+1$, the induced volume form $\alpha \wedge(d \alpha)^{n}$ is then preserved by $\left(\varphi^{t}\right)$, i.e. contact-Anosov flows are always conservative. For contact-Anosov flows of any odd dimension, $\left(\varphi^{t}\right)$ moreover preserves a natural linear connection on the tangent bundle, initially defined by Kanai in [Kan88]. An invariant connection of this kind allowed for example Benoist, Foulon and Labourie to obtain a classification result for contact-Anosov flows of any odd dimension in [BFL92]. 
While these invariant rigid geometric structures require the existence of a continuous one-parameter flow, we study in this paper rigid geometric structures preserved by discrete-time dynamics.

The transition from a flow to a diffeomorphism completely changes the situation. From a dynamical point of view, partially hyperbolic diffeomorphisms of "contact" type do not anymore preserve a contact one-form, and are thus (a priori) not conservative (which explains the extra hypothesis on non-wandering points). From a geometrical point of view, the difficulties that appear are analog to the ones of a conformal geometry in contrast with a metric geometry, for example the invariant Kanai connection does not anymore exist. This situation requires to look for a new rigid geometric structure.

A contact plane distribution is far from being rigid: according to Darboux's theorem, they are all locally isomorphic. A single smooth one-dimensional distribution in a contact plane distribution is still not sufficient to make it rigid. But if the stable and unstable distributions of the partially hyperbolic diffeomorphism are smooth and of contact sum, then the pair $\left(E^{s}, E^{u}\right)$ is a rigid geometric structure, called a Lagrangian contact structure.

For this structure, the invariant Kanai connection will be replaced by another type of connection called a Cartan connection, that defines a Cartan geometry (actually, this Cartan geometry partially appears in [Ghy87], but under the disguised form of "the geometry of second-order ordinary differential equations"). The strength of Cartan geometries is to link the Lagrangian contact structures with the homogeneous model space $\mathbf{X}=\mathrm{PGL}_{3}(\mathbb{R}) / \mathbf{P}_{\text {min }}$ of complete flags of $\mathbb{R}^{3}$ (where $\mathbf{P}_{\text {min }}$ is the subgroup of uppertriangular matrices). In particular, the flat Lagrangian contact structures, i.e. the ones whose curvature identically vanishes, are locally isomorphic to $\mathbf{X}$ (see Paragraphs 2.2.2 and 2.3.2). The geometry of $\mathbf{X}$ will thus play a prominent role in this paper.

In [Bar10], Barbot also studies the geometry of $\mathbf{X}$ and the dynamics of $\mathrm{PGL}_{3}(\mathbb{R})$, but with a different approach. His purpose is among others to construct Anosov representations in $\mathrm{PGL}_{3}(\mathbb{R})$, and compact quotients of open subsets of $\mathbf{X}$.

1.3. Organization of the paper. This paper is organised in the following way. Section 2 introduces several notions and results about three-dimensional Lagrangian contact structures, that will be used in the whole paper. At the end of the paper in Paragraph 8.2, we prove Theorem A from Theorem B, and the rest of the paper is devoted to the proof of Theorem B. In Section 3, we begin this proof by showing that the triplet $\mathcal{S}=\left(E^{\alpha}, E^{\beta}, E^{c}\right)$ is quasi-homogeneous, i.e. locally homogeneous in restriction to a dense open subset $\Omega$ of $M$, and that its isotropy on $\Omega$ is non-trivial. This implies that the Lagrangian contact structure $\left(E^{\alpha}, E^{\beta}\right)$ is flat, i.e. that $M$ has a $\left(\mathrm{PGL}_{3}(\mathbb{R}), \mathbf{X}\right)$ structure. In Section 4, we refine this description, proving that $\left.\mathcal{S}\right|_{\Omega}$ is locally isomorphic to one of two possible homogeneous models $\left(Y_{\mathbf{t}}, \mathcal{S}_{\mathbf{t}}\right)$ or $\left(Y_{\mathbf{a}}, \mathcal{S}_{\mathbf{a}}\right)$. This relies on a technical classification of the underlying infinitesimal model, done in Section 5. A critical step is to show in Section 6 that the open dense subset $\Omega$ is actually equal to $M$, implying that $M$ has a $(H, Y)$-structure, with two possible models $\left(H_{\mathbf{t}}, Y_{\mathbf{t}}\right)$ or $\left(H_{\mathbf{a}}, Y_{\mathbf{a}}\right)$. We prove in Section 7 that this $(H, Y)$-structure is complete, implying that $(M, \mathcal{S})$ is a compact quotient $\Gamma \backslash Y$ of one of these two models, with $\Gamma$ a discrete subgroup of $H=\operatorname{Aut}(Y)$. This description allows us to conclude the proof of Theorem B in Paragraph 8.1. 
Conventions and notations. From now on, every differential geometric object will be supposed to be $\operatorname{smooth}\left(\right.$ i.e. $\left.\mathcal{C}^{\infty}\right)$ if nothing is precised, and the manifolds will be supposed to be boundaryless.

The flow of a vector field $X$ is denoted by $\left(\varphi_{X}^{t}\right)$. The Lie algebra of a Lie group $G$ is denoted by $\mathfrak{g}$, and for any $v \in \mathfrak{g}$, we denote by $\tilde{v}$ the left-invariant vector field of $G$ generated by $v$. If $\Theta: G \times M \rightarrow M$ is a smooth group action (on the left or the right) of $G$ on a manifold $M$, then the orbital map of the action at $x \in M$ is denoted by $\theta_{x}=\Theta(\cdot, x)$, and we denote by $L_{g}=\Theta(g, \cdot)$ the translation by $g \in G$ if the action is on the left (respectively by $R_{g}$ if the action is on the right). For any $v \in \mathfrak{g}$ we denote by $v^{\dagger}$ the fundamental vector field of the action generated by $v$, defined for $x \in M$ by $v^{\dagger}(x)=\mathrm{D}_{e} \theta_{x}(v)$.

Acknowledgments. I would like to thank Charles Frances for proposing this subject to me, and for the precious advices that he offers me.

\section{Three-Dimensional Lagrangian CONTACT StRUCtURES}

The rigid geometric structures that will be studied in the rest of this paper are the following.

Definition 2.1. A Lagrangian contact structure $\mathcal{L}$ on a three-dimensional manifold $M$ is a pair $\mathcal{L}=\left(E^{\alpha}, E^{\beta}\right)$ of transverse one-dimensional smooth distributions, such that $E^{\alpha} \oplus E^{\beta}$ is a contact distribution. An enhanced Lagrangian contact structure $\mathcal{S}$ on $M$ is a triplet $\mathcal{S}=\left(E^{\alpha}, E^{\beta}, E^{c}\right)$ of one-dimensional smooth distributions such that $\mathrm{T} M=E^{\alpha} \oplus E^{\beta} \oplus E^{c}$, and $E^{\alpha} \oplus E^{\beta}$ is a contact distribution.

A (local) isomorphism between two Lagrangian contact structures is a (local) diffeomorphism that individually preserves the distributions $\alpha$ and $\beta$, and the (local) isomorphisms of enhanced Lagrangian contact structures preserve in addition the central distribution $E^{c}$.

We first define what will be for us the most important example of three-dimensional Lagrangian contact structure.

2.1. Homogeneous model space. We will call projective line the projection in $\mathbb{R} \mathbf{P}^{2}$ of a plane of $\mathbb{R}^{3}$, and we denote by $\mathbb{R} \mathbf{P}_{*}^{2}$ the set of projective lines of $\mathbb{R} \mathbf{P}^{2}$ (called the dual projective plane). For any subset $Q$ of $\mathbb{R}^{n+1}$ we denote by $[Q]$ the projection in $\mathbb{R} \mathbf{P}^{n}$ of the linear subspace of $\mathbb{R}^{n+1}$ generated by $Q$. by

A pointed projective line is a pair $(m, D)$ with $D \in \mathbb{R} \mathbf{P}_{*}^{2}$ and $m \in D$, and we denote

$$
\mathbf{X}=\left\{(m, D) \mid D \in \mathbb{R} \mathbf{P}_{*}^{2}, m \in D\right\} \subset \mathbb{R} \mathbf{P}^{2} \times \mathbb{R} \mathbf{P}_{*}^{2}
$$

the space of pointed projective lines. In other words, $\mathbf{X}$ is the space of complete flags of $\mathbb{R}^{3}$. We will denote in the whole paper by

$$
\mathbf{G}=\mathrm{PGL}_{3}(\mathbb{R})
$$

the group of projective transformations of $\mathbb{R} \mathbf{P}^{2}$. As the projective action of $\mathbf{G}$ on $\mathbb{R} \mathbf{P}^{2}$ and $\mathbb{R} \mathbf{P}_{*}^{2}$ preserves the incidence relation $m \in D$, it induces a natural diagonal action of 
$\mathbf{G}$ on $\mathbf{X} \subset \mathbb{R} \mathbf{P}^{2} \times \mathbb{R} \mathbf{P}_{*}^{2}$. The action of $\mathbf{G}$ on $\mathbf{X}$ is transitive, and the stabilizer in $\mathbf{G}$ of the base-point $o=\left(\left[e_{1}\right],\left[e_{1}, e_{2}\right]\right)$ of $\mathbf{X}$ is the subgroup

$$
\operatorname{Stab}_{\mathbf{G}}(o)=\mathbf{P}_{\text {min }}=\left\{\left[\begin{array}{lll}
* & * & * \\
0 & * & * \\
0 & 0 & *
\end{array}\right]\right\}<\mathbf{G}
$$

of upper-triangular matrices. From now on, we will identify $\mathbf{X}$ and $\mathbf{G} / \mathbf{P}_{\min }$ by the orbital map $\bar{\theta}_{o}: \mathbf{G} / \mathbf{P}_{\min } \rightarrow \mathbf{X}$ at $o$. The homogeneous space $\mathbf{X}$ is a $\mathbb{R} \mathbf{P}^{1}$-bundle over $\mathbb{R} \mathbf{P}^{2}$ and $\mathbb{R} \mathbf{P}_{*}^{2}$ through the coordinate projections

$$
\pi_{\alpha}:(m, D) \in \mathbf{X} \mapsto m \in \mathbb{R} \mathbf{P}^{2} \text { and } \pi_{\beta}:(m, D) \in \mathbf{X} \mapsto D \in \mathbb{R} \mathbf{P}_{*}^{2} .
$$

For $x=(m, D) \in \mathbf{X}$, we will denote by $\mathcal{C}^{\alpha}(x)=\mathcal{C}^{\alpha}(m)$ (respectively $\left.\mathcal{C}^{\beta}(x)=\mathcal{C}^{\beta}(D)\right)$ the fiber of $x$ with respect to $\pi_{\alpha}$ (resp. $\pi_{\beta}$ ), and we will call it the $\alpha$-circle (resp. the $\beta$-circle) of $x$. We denote by

$$
\mathcal{E}^{\alpha}=\operatorname{Ker}\left(\mathrm{D} \pi_{\alpha}\right) \text { and } \mathcal{E}^{\beta}=\operatorname{Ker}\left(\mathrm{D} \pi_{\beta}\right),
$$

the one-dimensional vertical distributions of these bundles, tangent respectively to the foliations by $\alpha$ and $\beta$-circles. The sum $\mathcal{E}^{\alpha} \oplus \mathcal{E}^{\beta}$ is contact and we will call $\mathcal{L}_{\mathbf{X}}=\left(\mathcal{E}^{\alpha}, \mathcal{E}^{\beta}\right)$ the standard Lagrangian contact structure of $\mathbf{X}$.

Lemma 2.2. The group $\mathbf{G}$ is the group of automorphisms of the standard Lagrangian contact structure $\mathcal{L}_{\mathbf{X}}$. In particular, the structure $\left(\mathbf{X}, \mathcal{L}_{\mathbf{X}}\right)$ is homogeneous.

Proof. First of all, the action of $\mathbf{G}$ preserves the foliations of $\mathbf{X}$ by $\alpha$ and $\beta$-circles, i.e. preserves the structure $\mathcal{L}_{\mathbf{X}}=\left(\mathcal{E}^{\alpha}, \mathcal{E}^{\beta}\right)$. Conversely, if $f$ is a diffeomorphism of $\mathbf{X}$ that preserves $\mathcal{L}_{\mathbf{X}}$, the fact that $f$ preserves the foliation by $\alpha$-circles simply means that it induces a diffeomorphism $\bar{f}$ of $\mathbb{R} \mathbf{P}^{2}$ for which $f$ is a lift through the projection $\pi_{\alpha}$. As $f$ moreover preserves the foliation by $\beta$-circles, $\bar{f}$ maps any projective line to a projective line. This implies that $\bar{f}$ is a projective transformation according to a classical result of projective geometry (proved for example in [Sam89, Theorem 7 p.32]), i.e. that $f$ is induced by the action of an element of $\mathbf{G}$.

2.2. Lagrangian contact structures as Cartan geometries. We now introduce the Cartan geometries modelled on the homogeneous space $\mathbf{G} / \mathbf{P}_{\text {min }}$, and make the link with Lagrangian contact structures. This notion will be our principal technical tool to deal with Lagrangian contact structures. We refer the reader to [Sha97] or [ČS09] for further details about Cartan geometries in a more general context.

\subsubsection{Cartan geometries modelled on $\mathbf{G} / \mathbf{P}_{\min }$.}

Definition 2.3. A Cartan geometry $\mathcal{C}=(\hat{M}, \omega)$ modelled on $\mathbf{G} / \mathbf{P}_{\text {min }}$ on a threedimensional manifold $M$ is the data of a $\mathbf{P}_{m i n}$-principal bundle over $M$ denoted by $\pi: \hat{M} \rightarrow M$ and called the Cartan bundle, together with a $\mathfrak{s l}_{3}$-valued one-form $\omega: \mathrm{T} \hat{M} \rightarrow$ $\mathfrak{s l}_{3}$ on $\hat{M}$ called the Cartan connection, that satisfies the three following properties:

(1) $\omega$ defines a parallelism of $\hat{M}$, i.e. for any $\hat{x} \in \hat{M}, \omega_{\hat{x}}$ is a linear isomorphism from $\mathrm{T}_{\hat{x}} \hat{M}$ to $\mathfrak{s l}_{3}$,

(2) $\omega$ reproduces the fundamental vector fields of the right action of $\mathbf{P}_{\text {min }}$, i.e. for any $v \in \mathfrak{s l}_{3}$ and $\hat{x} \in \hat{M}$ we have: $v^{\dagger}(\hat{x})=\left.\frac{d}{d t}\right|_{t=0} \hat{x} \cdot \mathrm{e}^{t v}=\omega_{\hat{x}}^{-1}(v)$, 


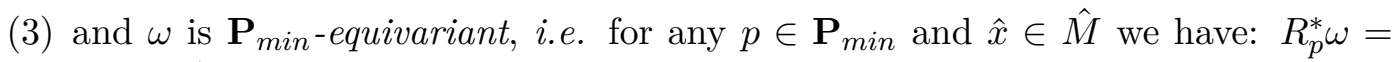
$\operatorname{Ad}(p)^{-1} \circ \omega$ (where $\operatorname{Ad}(p)$ stands for the adjoint action of $p$ ).

A (local) automorphism $f$ of the Cartan geometry $\mathcal{C}$ between two open sets $U$ and $V$ of $M$ is a (local) diffeomorphism from $U$ to $V$ that lifts to a $\mathbf{P}_{m i n}$-equivariant (local) diffeomorphism $\hat{f}$ between $\pi^{-1}(U)$ and $\pi^{-1}(V)$, such that $\hat{f}$ preserves the Cartan connexion $\omega\left(\right.$ i.e. $\left.\hat{f}^{*} \omega=\omega\right)$.

Example 2.4. The homogeneous model space $\mathbf{X}$ is endowed with the Cartan geometry of the model $\mathcal{C}_{\mathbf{X}}=\left(\mathbf{G}, \omega_{\mathbf{G}}\right)$, given by the canonical $\mathbf{P}_{\text {min }}$-bundle $\pi_{\mathbf{G}}: \mathbf{G} \rightarrow \mathbf{G} / \mathbf{P}_{\min }=\mathbf{X}$ over $\mathbf{X}$, together with the Maurer-Cartan one-form $\omega_{\mathbf{G}}: \mathbf{T} \mathbf{G} \rightarrow \mathfrak{s l}_{3}$ defined by $\omega_{\mathbf{G}}(\tilde{v}) \equiv v$ on the left-invariant vector fields of $\mathbf{G}$ ).

We consider for the rest of the subsection a Cartan geometry $(M, \mathcal{C})=(M, \hat{M}, \omega)$ modelled on $\mathbf{G} / \mathbf{P}_{\min }$.

2.2.2. Curvature of a Cartan geometry. The following definition replaces the curvature of a Riemannian metric in the case of Cartan geometries.

Definition 2.5. The curvature form of $\mathcal{C}$ is the $\mathfrak{s l}_{3}$-valued two-form $\Omega$ of $\hat{M}$ defined by the following relation for two vector fields $X$ and $Y$ on $\hat{M}$ :

$$
\Omega(X, Y)=d \omega(X, Y)+[\omega(X), \omega(Y)] .
$$

Thanks to the connection $\omega$, the curvature form $\Omega$ is equivalent to a curvature map $K: \hat{M} \rightarrow \mathrm{L}\left(\Lambda^{2} \mathfrak{s l}_{3}, \mathfrak{s l}_{3}\right)$ on $\hat{M}$ (that we will often simply call the curvature of $\mathcal{C}$ ), having values in the vector space of $\mathfrak{s l}_{3}$-valued alternated bilinear maps on $\mathfrak{s l}_{3}$, and defined by the following relation for $\hat{x} \in \hat{M}$ and $v, w \in \mathfrak{s l}_{3}$ :

$$
K_{\hat{x}}(v, w)=\Omega\left(\omega_{\hat{x}}^{-1}(v), \omega_{\hat{x}}^{-1}(w)\right) .
$$

We will say that the Cartan geometry $\mathcal{C}$ (or the Cartan connection $\omega$ ) is torsion-free if $K_{\hat{x}}(v, w) \in \mathfrak{p}_{\text {min }}$ for any $\hat{x} \in \hat{M}$ and $v, w \in \mathfrak{s l}_{3}$.

If $v$ or $w$ is tangent to the fiber of the principal bundle $\hat{M}$, then the curvature form satisfies $\Omega(v, w)=0$ (this is proved in [Sha97, Chapter 5 Corollary 3.10]). As $\omega$ maps the tangent space of the fibers to $\mathfrak{p}_{\text {min }}$ (because the fundamental vector fields are $\omega$ invariant), this implies that the curvature $K(v, w)$ vanishes whenever $v$ or $w$ is in $\mathfrak{p}_{\text {min }}$. As a consequence at any point $\hat{x} \in \hat{M}, K_{\hat{x}}$ induces a $\mathfrak{s l}_{3}$-valued alternated bilinear map on $\mathfrak{s l}_{3} / \mathfrak{p}_{\text {min }}$, and we will identify in the sequel $K$ with the induced map

$$
K: \hat{M} \rightarrow \mathrm{L}\left(\Lambda^{2}\left(\mathfrak{s l}_{3} / \mathfrak{p}_{\text {min }}\right), \mathfrak{s l}_{3}\right) .
$$

The adjoint action of $\mathbf{P}_{\text {min }}$ induces a linear left action on $\mathrm{L}\left(\Lambda^{2}\left(\mathfrak{s l}_{3} / \mathfrak{p}_{\text {min }}\right), \mathfrak{s l}_{3}\right)$ defined for $p \in \mathbf{P}_{\text {min }}$ and $K \in \mathrm{L}\left(\Lambda^{2}\left(\mathfrak{s l}_{3} / \mathfrak{p}_{\text {min }}\right), \mathfrak{s l}_{3}\right)$ by

$$
p \cdot K: u \wedge v \mapsto \operatorname{Ad}(p) \cdot\left(K\left(\overline{\operatorname{Ad}}(p)^{-1} \cdot u, \overline{\operatorname{Ad}}(p)^{-1} \cdot v\right)\right) .
$$

Using the linear right action of $\mathbf{P}_{\text {min }}$ on $\mathrm{L}\left(\Lambda^{2}\left(\mathfrak{s l}_{3} / \mathfrak{p}_{\text {min }}\right), \mathfrak{s l}_{3}\right)$ defined by $K \cdot p:=p^{-1} \cdot K$, $K$ is $\mathbf{P}_{m i n}$-equivariant (this is proved in [Sha97, Chapter 5 Lemma 3.23]), and $K$ is moreover preserved by any local automorphism $f$ of the Cartan geometry (i.e $K \circ \hat{f}=K$ for any automorphism). 
2.2.3. Lagrangian contact structure induced by a Cartan geometry. At any point $x \in M$ and for any $\hat{x} \in \pi^{-1}(x)$, we denote by $i_{\hat{x}}: \mathrm{T}_{x} M \rightarrow \mathfrak{s l}_{3} / \mathfrak{p}_{\text {min }}$ the unique isomorphism satisfying

$$
i_{\hat{x}} \circ \mathrm{D}_{\hat{x}} \pi=\bar{\omega}_{\hat{x}}
$$

where $\bar{\omega}$ denotes the projection of $\omega$ on $\mathfrak{s l}_{3} / \mathfrak{p}_{\text {min }}$. As the adjoint action of $\mathbf{P}_{\text {min }}$ preserves $\mathfrak{p}_{\text {min }}$, it induces a representation $\overline{\mathrm{Ad}}: \mathbf{P}_{\text {min }} \rightarrow \mathrm{GL}\left(\mathfrak{s l}_{3} / \mathfrak{p}_{\text {min }}\right)$ on the quotient, and the equivariance of $\omega$ implies the following relation for any $p \in \mathbf{P}_{\min }$ :

$$
i_{\hat{x} \cdot p}=\overline{\operatorname{Ad}}(p)^{-1} \circ i_{\hat{x}} .
$$

This relation shows that any $\overline{\operatorname{Ad}}\left(\mathbf{P}_{\text {min }}\right)$-invariant object on $\mathfrak{s l}_{3} / \mathfrak{p}_{\text {min }}$ gives rise, through the isomorphisms $i_{\hat{x}}$, to a well-defined object on the tangent bundle of $M$. Let us apply this idea to define a Lagrangian contact structure on $M$ associated to the Cartan geometry $\mathcal{C}$. We introduce

$$
e_{\alpha}=\left(\begin{array}{lll}
0 & 0 & 0 \\
0 & 0 & 0 \\
0 & 1 & 0
\end{array}\right), e_{\beta}=\left(\begin{array}{lll}
0 & 0 & 0 \\
1 & 0 & 0 \\
0 & 0 & 0
\end{array}\right), e_{0}=\left(\begin{array}{lll}
0 & 0 & 0 \\
0 & 0 & 0 \\
1 & 0 & 0
\end{array}\right),
$$

defining a basis $\left(\bar{e}_{\alpha}, \bar{e}_{\beta}, \bar{e}_{0}\right)$ of $\mathfrak{s l}_{3} / \mathfrak{p}_{\text {min }}$, in which the matrix of the adjoint action of

$$
p=\left(\begin{array}{ccc}
a & x & z \\
0 & a^{-1} b^{-1} & y \\
0 & 0 & b
\end{array}\right) \in \mathbf{P}_{\text {min }}
$$

is equal to

$$
\operatorname{Mat}_{\left(\bar{e}_{\alpha}, \bar{e}_{\beta}, \bar{e}_{0}\right)}(\overline{\operatorname{Ad}}(p))=\left(\begin{array}{ccc}
a^{-2} b^{-1} & 0 & a^{-1} y \\
0 & a b^{2} & -b^{2} x \\
0 & 0 & a^{-1} b
\end{array}\right) .
$$

In particular, the adjoint action of $\mathbf{P}_{\text {min }}$ individually preserves the lines $\mathbb{R} \bar{e}_{\alpha}$ and $\mathbb{R} \bar{e}_{\beta}$ of $\mathfrak{s l}_{3} / \mathfrak{p}_{\text {min }}$. Together with the relation (2.7), this shows that for $x \in M$, the lines $i_{\hat{x}}^{-1}\left(\mathbb{R} \bar{e}_{\alpha}\right)$ and $i_{\hat{x}}^{-1}\left(\mathbb{R}_{\beta}\right)$ of $\mathrm{T}_{x} M$ do not depend on the lift $\hat{x}$ of $x$. The Cartan geometry $\mathcal{C}$ induces thus two one-dimensional distributions $E_{\mathcal{C}}^{\alpha}(x)=i_{\hat{x}}^{-1}\left(\mathbb{R} \bar{e}_{\alpha}\right)$ and $E_{\mathcal{C}}^{\beta}(x)=i_{\hat{x}}^{-1}\left(\mathbb{R} \bar{e}_{\beta}\right)$ on $M$, and the curvature of $\mathcal{C}$ will say when do those distributions define a Lagrangian contact structure.

Lemma 2.6. Any torsion-free Cartan geometry $(M, \mathcal{C})$ modelled on $\mathbf{G} / \mathbf{P}_{\text {min }}$ induces a Lagrangian contact structure $\left(E_{\mathcal{C}}^{\alpha}, E_{\mathcal{C}}^{\beta}\right)$ on the three-dimensional base manifold $M$.

Sketch of proof. For $x \in M$, considering a local section of the Cartan bundle over $x$, we can push down by $\pi$ the $\omega$-constant vector fields $\tilde{e}_{\alpha}$ and $\tilde{e}_{\beta}$ of $\hat{M}$ (characterized by $\left.\omega\left(\tilde{e}_{\varepsilon}\right) \equiv e_{\varepsilon}\right)$ to local vector fields $X_{\alpha}$ and $X_{\beta}$ of $M$ defined on a neighbourhood of $x$, that respectively generate the distributions $E_{\mathcal{C}}^{\alpha}$ and $E_{\mathcal{C}}^{\beta}$. If $K$ has values in $\mathfrak{p}_{\text {min }}$, the identity $\omega\left(\left[\tilde{e}_{\alpha}, \tilde{e}_{\beta}\right]\right)=\left[e_{\alpha}, e_{\beta}\right]-K\left(e_{\alpha}, e_{\beta}\right)$ (deduced from Cartan's formula for the differential of a one-form) implies easily that $\left[X_{\alpha}, X_{\beta}\right] \notin \operatorname{Vect}\left(X_{\alpha}, X_{\beta}\right)$ in the neighbourhood of $x$, finishing the proof.

Remark 2.7. In the case of the Cartan geometry of the model, it is easy to check that $\left(E_{\mathcal{C}_{\mathbf{X}}}^{\alpha}, E_{\mathcal{C}_{\mathbf{X}}}^{\beta}\right)$ is the standard Lagrangian contact structure $\mathcal{L}_{\mathbf{X}}$ of $\mathbf{X}$. 
2.3. Normal Cartan geometry of a Lagrangian contact structure. Any threedimensional Lagrangian contact structure is actually induced by a torsion-free Cartan geometry modelled on $\mathbf{G} / \mathbf{P}_{\text {min }}$. This equivalence between three-dimensional Lagrangian contact structures and Cartan geometries modelled on $\mathbf{G} / \mathbf{P}_{\min }$ was discovered by Élie Cartan, who developped this notion and after whom these geometries are named.

2.3.1. Equivalence problem for Lagrangian contact structures. A given three-dimensional Lagrangian contact structure is induced by several Cartan connections, and to obtain an equivalence between both formulations, we have to choose a particular one. This choice will be done through a normalisation condition on the curvature. Using the basis $\left(\bar{e}_{\alpha} \wedge \bar{e}_{0}, \bar{e}_{\beta} \wedge \bar{e}_{0}, \bar{e}_{\alpha} \wedge \bar{e}_{\beta}\right)$ of $\Lambda^{2}\left(\mathfrak{s l}_{3} / \mathfrak{p}_{\text {min }}\right)$, we consider the following four-dimensional subspace of $\mathrm{L}\left(\Lambda^{2}\left(\mathfrak{s l}_{3} / \mathfrak{p}_{\min }\right), \mathfrak{s l}_{3}\right)$ :

$$
W_{K}=\left\{K: \bar{e}_{\alpha} \wedge \bar{e}_{0} \mapsto\left(\begin{array}{ccc}
0 & 0 & K^{\alpha} \\
0 & 0 & K_{\alpha} \\
0 & 0 & 0
\end{array}\right), \bar{e}_{\beta} \wedge \bar{e}_{0} \mapsto\left(\begin{array}{ccc}
0 & K_{\beta} & K^{\beta} \\
0 & 0 & 0 \\
0 & 0 & 0
\end{array}\right), \bar{e}_{\alpha} \wedge \bar{e}_{\beta} \mapsto 0\right\} .
$$

The linear action of $\mathbf{P}_{\text {min }}$ preserves $W_{K}$, that will be called the space of normal curvatures. Theorem 2.8 below is proved in [DK16, Theorem 3 p.14], where the normalisation condition is explicitely calculated through Cartan's method of equivalence (see also [ČS09, Theorem 3.1.14 p.271 and Paragraph 4.2.3] that makes the link with general parabolic Cartan geometries).

Theorem 2.8 (E. Cartan, [DK16], [ČS09]). For any Lagrangian contact structure $\mathcal{L}$ on a three-dimensional manifold $M$, there exists a torsion-free Cartan geometry modelled on $\mathbf{G} / \mathbf{P}_{\min }$ inducing $\mathcal{L}$ on $M$, and whose curvature map has values in the space $W_{K}$ of normal curvatures. Such a Cartan geometry is unique (up to action of principal bundle automorphisms covering the identity on $M$ ), and will be called the normal Cartan geometry of $\mathcal{L}$.

Furthermore, if $\left(M_{1}, \mathcal{L}_{1}\right)$ and $\left(M_{2}, \mathcal{L}_{2}\right)$ are two three-dimensional Lagrangian contact structures, and $\mathcal{C}_{1}, \mathcal{C}_{2}$ are the associated normal Cartan geometries, then the (local) isomorphisms between $\mathcal{L}_{1}$ and $\mathcal{L}_{2}$ and the (local) isomorphism between $\mathcal{C}_{1}$ and $\mathcal{C}_{2}$ are the same. This a direct consequence of the unicity of the normal Cartan geometry. The curvature map $K: \hat{M} \rightarrow W_{K}$ of the normal Cartan geometry of a three-dimensional Lagrangian contact structure $\mathcal{L}$ will simply be called the curvature of $\mathcal{L}$.

2.3.2. Flat Lagrangian contact structures. The homogeneous model space $\left(\mathbf{X}, \mathcal{L}_{\mathbf{X}}\right)$ verifies the following analog of Liouville's theorem.

Theorem 2.9. For any connected open subsets $U$ and $V$ of the homogeneous model space $\mathbf{X}$, and any diffeomorphism $f$ from $U$ to $V$ that preserves its standard Lagrangian contact structure $\mathcal{L}_{\mathbf{X}}$, there exists $g \in \mathbf{G}$ such that $f$ is the restriction to $U$ of the translation by $g$.

Proof. The Maurer-Cartan form $\omega_{\mathbf{G}}$ satisfies for any tangent vectors $v$ and $w$ the structural equation $d \omega_{\mathbf{G}}(v, w)+\left[\omega_{\mathbf{G}}(v), \omega_{\mathbf{G}}(w)\right]=0$ (see [Sha97, §3.3 p.108]), implying that the curvature of the Cartan connection $\omega_{\mathbf{G}}$ is zero. Therefore, the curvature satisfies the normalisation condition of Theorem 2.8 , and $\mathcal{C}_{\mathbf{X}}$ is a normal Cartan geometry on $\mathbf{X}$ modelled on $\mathbf{G} / \mathbf{P}_{\text {min }}$ and associated to $\mathcal{L}_{\mathbf{X}}$ (see Remark 2.7). According to Theorem 2.8 , any local isomorphism of $\mathcal{L}_{\mathbf{X}}$ between two connected open subset $U$ and $V$ of $\mathbf{X}$ 
lifts therefore to a local isomorphism of the Cartan geometry $\mathcal{C}_{\mathbf{X}}$ between $\pi_{\mathbf{G}}^{-1}(U)$ and $\pi_{\mathbf{G}}^{-1}(V)$, and such an automorphism is the left translation by an element of $\mathbf{G}$ according to [Sha97, Chapter 5 Theorem 5.2].

A three-dimensional Lagrangian contact structure $(M, \mathcal{L})$ is flat if the curvature of the normal Cartan geometry of $\mathcal{L}$ vanishes identically. According to the proof of Theorem 2.9 , the model space is flat, and since this property is local, any Lagrangian contact structure locally isomorphic to $\left(\mathbf{X}, \mathcal{L}_{\mathbf{X}}\right)$ is flat.

The power of Cartan geometries lies in the converse of this statement: any flat threedimensional Lagrangian contact structure $\mathcal{L}$ is locally isomorphic to the homogeneous model space (see [Sha97, Theorem 5.1 and Theorem 5.2 p. 212]). There exists in this case an atlas of charts from $M$ to $\mathbf{X}$ consisting of local isomorphisms of Lagrangian contact structures from $\mathcal{L}$ to $\mathcal{L}_{\mathbf{X}}$, and whose transition maps are restrictions of left translations by elements of $\mathbf{G}$ (according to Theorem 2.9). A maximal atlas satisfying these conditions is called a $(\mathbf{G}, \mathbf{X})$-structure on $M$. Any $(\mathbf{G}, \mathbf{X})$-structure conversely induces on $M$ a Lagrangian contact structure $\mathcal{L}$ locally isomorphic to $\mathcal{L}_{\mathbf{X}}$, whose charts are local isomorphisms from $\mathcal{L}$ to $\mathcal{L}_{\mathbf{X}}$.

Theorem 2.10. Any flat three-dimensional Lagrangian contact structure $(M, \mathcal{L})$ is induced by a $(\mathbf{G}, \mathbf{X})$-structure on $M$.

Denoting by $\pi_{M}: \tilde{M} \rightarrow M$ the universal cover of $M$, we recall that any $(\mathbf{G}, \mathbf{X})$ structure on $M$ is described by a local diffeomorphism $\delta: \tilde{M} \rightarrow \mathbf{X}$ called the developping map, that is equivariant for a morphism $\rho: \pi_{1}(M) \rightarrow \mathbf{G}$ called the holonomy morphism (see for example [Thu97, §3.4 p.139-141]). Moreover for any $g \in \mathbf{G}$, the pair $\left(g \circ \delta, g \rho g^{-1}\right)$ of developping map and holonomy morphism describes the same $(\mathbf{G}, \mathbf{X})$ structure. The Lagrangian contact structure $\mathcal{L}$ induced by a $(\mathbf{G}, \mathbf{X})$-structure is characterized by: $\delta^{*} \mathcal{L}_{\mathbf{X}}=\pi_{M}^{*} \mathcal{L}$.

2.3.3. Harmonic curvature. For $K \in W_{K}$ an element of the space of normal curvatures defined by

and

$$
K: \bar{e}_{\alpha} \wedge \bar{e}_{0} \mapsto\left(\begin{array}{ccc}
0 & 0 & K^{\alpha} \\
0 & 0 & K_{\alpha} \\
0 & 0 & 0
\end{array}\right), \bar{e}_{\beta} \wedge \bar{e}_{0} \mapsto\left(\begin{array}{ccc}
0 & K_{\beta} & K^{\beta} \\
0 & 0 & 0 \\
0 & 0 & 0
\end{array}\right), \bar{e}_{\alpha} \wedge \bar{e}_{\beta} \mapsto 0,
$$

$$
p=\left(\begin{array}{ccc}
a & x & z \\
0 & a^{-1} b^{-1} & y \\
0 & 0 & b
\end{array}\right) \in \mathbf{P}_{\text {min }},
$$

the adjoint action (2.9) of $\mathbf{P}_{\min }$ given in Paragraph 2.3 enables to compute the components $\cdot_{\alpha}$ and $\cdot \beta$ of $p \cdot K \in W_{K}$ :

$$
(p \cdot K)_{\alpha}=a^{-1} b^{-5} K_{\alpha},(p \cdot K)_{\beta}=a^{5} b K_{\beta} .
$$

These expressions show in particular that the two-dimensional subspace $W_{H}=\{K \in$ $\left.W_{K} \mid K_{\alpha}=K_{\beta}=0\right\}$ of $W_{K}$ is preserved by the linear action of $\mathbf{P}_{\min }$.

Proposition 2.11. If the curvature map of a three-dimensional Lagrangian contact structure $\mathcal{L}$ has values in the subspace $W_{H}$ (i.e. if $K_{\alpha}$ and $K_{\beta}$ identically vanish), then $\mathcal{L}$ is flat. 
The following remark will be useful in the proof of this result: $\mathfrak{s l}_{3}$ is a two-graded Lie algebra, the graduation being defined by the following subspaces $\left(\mathfrak{s l}_{3}\right)_{i}$ for $i=-2, \cdots, 2$ :

$$
\mathfrak{s l}_{3}=\left(\begin{array}{ccc}
\left(\mathfrak{s l}_{3}\right)_{0} & \left(\mathfrak{s l}_{3}\right)_{1} & \left(\mathfrak{s l}_{3}\right)_{2} \\
\left(\mathfrak{s l}_{3}\right)_{-1} & \left(\mathfrak{s l}_{3}\right)_{0} & \left(\mathfrak{s l}_{3}\right)_{1} \\
\left(\mathfrak{s l}_{3}\right)_{-2} & \left(\mathfrak{s l}_{3}\right)_{-1} & \left(\mathfrak{s l}_{3}\right)_{0}
\end{array}\right) .
$$

The graduation property of $\mathfrak{s l}_{3}$ simply means that for any $i$ and $j$ we have $\left[\left(\mathfrak{s l}_{3}\right)_{i},\left(\mathfrak{s l}_{3}\right)_{j}\right] \subset$ $\left(\mathfrak{s l}_{3}\right)_{i+j}$, (where $\left(\mathfrak{s l}_{3}\right)_{i}=\{0\}$ for any $\left.|i|>2\right)$. This graduation of $\mathfrak{s l}_{3}$ gives rise to a filtration defined by $\mathfrak{s l}_{3}{ }^{i}=\oplus_{j \geq i}\left(\mathfrak{s l}_{3}\right)_{j}$, with respect to which $\mathfrak{s l}_{3}$ is a filtered Lie algebra, i.e. $\left[\mathfrak{s l}_{3}{ }^{i}, \mathfrak{s l}_{3}{ }^{j}\right] \subset \mathfrak{s l}_{3}{ }^{i+j}\left(\right.$ with $\mathfrak{s l}_{3}{ }^{i}=\mathfrak{s l}_{3}$ for $i \leq-2$ and $\mathfrak{s l}_{3}{ }^{i}=\{0\}$ for $i>2$ ).

Proof of Proposition 2.11. Let $(M, \hat{M}, \omega)$ be a normal Cartan geometry modelled on $\mathbf{G} / \mathbf{P}_{\text {min }}$. We introduce the following basis of $\mathfrak{s l}_{3}$ :

$e_{0}=\left(\begin{array}{lll}0 & 0 & 0 \\ 0 & 0 & 0 \\ 1 & 0 & 0\end{array}\right), e_{\alpha}=\left(\begin{array}{lll}0 & 0 & 0 \\ 0 & 0 & 0 \\ 0 & 1 & 0\end{array}\right), e_{\beta}=\left(\begin{array}{lll}0 & 0 & 0 \\ 1 & 0 & 0 \\ 0 & 0 & 0\end{array}\right), e_{1}=\left(\begin{array}{ccc}1 & 0 & 0 \\ 0 & -1 & 0 \\ 0 & 0 & 0\end{array}\right), e_{2}=\left(\begin{array}{ccc}0 & 0 & 0 \\ 0 & 1 & 0 \\ 0 & 0 & -1\end{array}\right), e^{\alpha}=$ $\left(\begin{array}{lll}0 & 0 & 0 \\ 0 & 0 & 1 \\ 0 & 0 & 0\end{array}\right), e^{\beta}=\left(\begin{array}{lll}0 & 1 & 0 \\ 0 & 0 & 0 \\ 0 & 0 & 0\end{array}\right), e^{0}=\left(\begin{array}{lll}0 & 0 & 1 \\ 0 & 0 & 0 \\ 0 & 0 & 0\end{array}\right)$, that we denote $\mathcal{B}$. We denote the coordinate of the Cartan connection $\omega$ with respect to an element $e$ of the basis $\mathcal{B}$ as a real-valued one-form $\omega_{e}$ on $\hat{M}$, such that $\omega=\sum_{e \in \mathcal{B}} \omega_{e} e$. In the same way, the curvature form $\Omega$ of $\omega$ will be denoted as $\Omega=\sum_{e \in \mathcal{B}} \Omega_{e} e$, where the $\Omega_{e}$ 's are real-valued two-forms on $\hat{M}$. According to the form (2.10) of the curvature map stated in Theorem 2.8, if $K_{\alpha}=K_{\beta}=0$ identically, then the only non-zero two-form $\Omega_{e}$ is $\Omega^{0}=K^{\alpha} \omega_{\alpha} \wedge \omega_{0}+K^{\beta} \omega_{\beta} \wedge \omega_{0}$. The Bianchi identity proved in [Sha97, Chapter 5 Lemma 3.30] gives $d \Omega=[\Omega, \omega]$, where $[\Omega, \omega]=L \circ(\Omega \wedge \omega)$ with $L: v \otimes w \in \mathfrak{s l}_{3} \otimes \mathfrak{s l}_{3} \mapsto[v, w] \in \mathfrak{s l}_{3}$ (see [Sha97, Chapter 1.5 p.61] for this definition). The graduation property of $\mathfrak{s l}_{3}$ exposed in the beginning of the paragraph implies $\left[e^{0}, \mathfrak{s l}_{3}^{+}\right]=\{0\}$, and we have the following Lie brackets relations between the elements of $\mathcal{B}:\left[e^{0}, e_{0}\right]=e_{1}+e_{2},\left[e^{0}, e_{\alpha}\right]=e^{\beta},\left[e^{0}, e_{\beta}\right]=-e^{\alpha}$, $\left[e^{0}, e_{1}\right]=\left[e^{0}, e_{2}\right]=-e^{0}$. We finally obtain the following equalities by projecting the Bianchi identity to $\mathbb{R} e^{\beta}$ and $\mathbb{R} e^{\alpha}$ :

$$
0=-K^{\alpha} \omega_{\alpha} \wedge \omega_{0} \wedge \omega_{\beta}, 0=K^{\beta} \omega_{\beta} \wedge \omega_{0} \wedge \omega_{\alpha} .
$$

As $\left(\omega_{\alpha}, \omega_{0}, \omega_{\beta}\right)$ is at each point $\hat{x} \in \hat{M}$ a basis of the dual space $\left.\left(\omega_{\hat{x}}^{-1}\left(\left(\mathfrak{s l}_{3}\right)_{-2} \oplus\left(\mathfrak{s l}_{3}\right)_{-1}\right)\right)\right)^{*}$, the three-form $\omega_{\alpha} \wedge \omega_{0} \wedge \omega_{\beta}$ does not vanish, and the above equalities imply therefore $K^{\alpha}=K^{\beta}=0$ identically, i.e. $K=0$ as announced.

Remark 2.12. The components $K_{\alpha}$ and $K_{\beta}$ of the curvature actually encode the harmonic curvature of a normal Cartan geometry modelled on $\mathbf{G} / \mathbf{P}_{\text {min }}$, that is known to be the only obstruction to the flatness for parabolic Cartan geometries. With this point of view, the above Proposition 2.11 is the manifestation in the specific case of Lagrangian contact structures of a general phenomena arising for any parabolic geometry (see for example [ČS09, Theorem 3.1.12]).

2.3.4. Normal generalized Cartan geometry of an enhanced Lagrangian contact structure. Let $\mathcal{S}=\left(E^{\alpha}, E^{\beta}, E^{c}\right)$ be an enhanced Lagrangian contact structure on a threedimensional manifold $M$, and $\mathcal{C}=(\hat{M}, \omega)$ be the normal Cartan geometry of the underlying Lagrangian contact structure $\left(E^{\alpha}, E^{\beta}\right)$. Using the isomorphisms $i_{\hat{x}}$ defined in 
(2.6), the transverse distribution $E^{c}$ is encoded by the map

$$
\varphi: \hat{x} \in \hat{M} \mapsto i_{\hat{x}}\left(E_{\pi(\hat{x})}^{c}\right) \in \mathbb{V},
$$

having values in the open subset

$$
\mathbb{V}=\left\{L \in \mathbf{P}\left(\mathfrak{s l}_{3} / \mathfrak{p}_{\text {min }}\right) \mid L \not \subset \operatorname{Vect}\left(\bar{e}_{\alpha}, \bar{e}_{\beta}\right)\right\}
$$

of $\mathbf{P}\left(\mathfrak{s l}_{3} / \mathfrak{p}_{\text {min }}\right)$. Endowing $\mathbb{V}$ with the right $\mathbf{P}_{\text {min }}$-action defined by $L \cdot p=\overline{\operatorname{Ad}}(p)^{-1}(L)$, $\varphi$ is $\mathbf{P}_{m i n}$-equivariant. Conversely, any $\mathbf{P}_{m i n}$-equivariant application $\varphi: \hat{M} \rightarrow \mathbb{V}$ defines a transverse distribution $E_{\pi(\hat{x})}^{c}=i_{\hat{x}}^{-1}(\varphi(\hat{x}))$ compatible with the Lagrangian contact structure $\left(E^{\alpha}, E^{\beta}\right)$.

Definition 2.13. $(\mathcal{C}, \varphi)=(\hat{M}, \omega, \varphi)$ will be called the normal generalized Cartan geometry of the enhanced Lagrangian contact structure $\mathcal{S}$.

\subsection{Killing fields of Lagrangian contact structures.}

2.4.1. Some classical properties of Killing fields. A (local) Killing field of a Lagrangian contact structure $(M, \mathcal{L})$ is a (local) vector field $X$ of $M$ whose flow preserves $\mathcal{L}$. The Killing fields of an enhanced Lagrangian contact structure $\mathcal{S}$ are defined in the same way. We will denote by $\mathfrak{K i t l}(U, \mathcal{L})$ the subalgebra of Killing fields of $\mathcal{L}$ defined on an open subset $U \subset M$, and by $\mathfrak{k i r l}_{\mathcal{L}}^{\text {loc }}(x)$ the Lie algebra of germs of Killing fields of $\mathcal{L}$ defined on a neighbourhood of $x$.

The following statement summarizes important properties of Killing fields, coming from their description through Cartan geometries and well-known in this context. The results are stated for Lagrangian contact structures, but are true as well for enhanced Lagrangian contact structures.

Lemma 2.14. Let $M$ be a three-dimensional connected manifold endowed with a Lagrangian contact structure $\mathcal{L}$, and $\mathcal{C}=(\hat{M}, \omega)$ be a normal Cartan geometry on $M$ associated to $\mathcal{L}$.

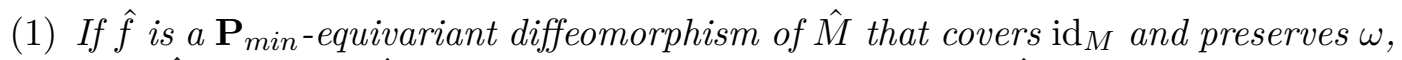

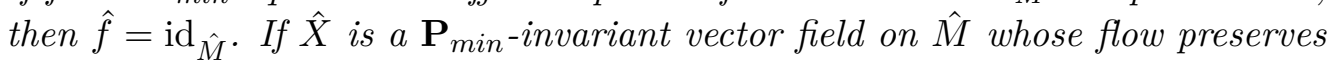
$\omega$ and whose projection on $M$ vanishes, then $\hat{X}=0$. As a consequence, the lift of a local automorphism $f$ (respectively Killing field $X$ ) of $\mathcal{L}$ to a $\mathbf{P}_{\text {min-equivariant }}$ diffeomorphism $\hat{f}$ of $\hat{M}$ that preserves $\omega$ (resp. to a $\mathbf{P}_{\text {min }}$-invariant vector field $\hat{X}$ on $\hat{M}$ whose flow preserves $\omega)$, is unique.

(2) If the lift $\hat{X}$ of a Killing field $X$ of $\mathcal{L}$ vanishes at some point $\hat{x}$, then $X=0$. In other words, the linear map $X \in \mathfrak{K i l l}(M, \mathcal{L}) \mapsto \omega_{\hat{x}}\left(\hat{X}_{\hat{x}}\right) \in \mathfrak{s l}_{3}$ is injective.

(3) The Lie algebra morphism $X \in \mathfrak{K i l l}(M, \mathcal{L}) \mapsto[X]_{x} \in \mathfrak{k i r l}_{\mathcal{L}}^{\text {loc }}(x)$ sending a Killing field of $\mathcal{L}$ to its germ at $x$ is injective.

Sketch of proof. 1. The first assertion is a direct consequence of [ČS09, Proposition 1.5.3] for Cartan geometries modelled on $\mathbf{G} / \mathbf{P}_{\text {min }}$, and implies the second one.

2. Let us assume that a local automorphism $\hat{f}$ of $\mathcal{C}$ fixes a point $\hat{x} \in \hat{M}$. Then as $\hat{f}$ preserves the parallelism defined by $\omega$, a classical argument implies that $\hat{f}$ is trivial on the connected component of $\hat{x}$. This remark easily implies the assertion about Killing fields. 
3. According to [BFM09, Lemma 7.1], a local automorphism that is trivial in the neighbourhood of $x$ is trivial on the connected component of its domain of definition that contains $x$. This result easily implies the statement concerning Killing fields.

Remark 2.15. The second statement of the previous lemma shows in particular that for any connected open neighbourhood $U$ of $x \in M$, the dimension of $\mathfrak{K i l l}(U, \mathcal{L})$ is bounded from above by $\operatorname{dim} \mathfrak{s l}_{3}=8$. Therefore, if we consider a decreasing sequence of connected open neighbourhoods $U_{i}$ of $x$ such that $\cap_{i} U_{i}=\{x\}$, then $\operatorname{dim} \mathfrak{K i l l}\left(U_{i}, \mathcal{L}\right)$ is constant for $i$ large enough. This proves the existence of a connected open neighbourhood $U$ of $x$ such that

$$
X \in \mathfrak{K i l r}(U, \mathcal{L}) \mapsto[X]_{x} \in \mathfrak{k i r l}_{\mathcal{L}}^{l o c}(x)
$$

is a Lie algebra isomorphism.

The following Lemma is the translation of Theorem 2.9 for Killing fields of $\left(\mathbf{X}, \mathcal{L}_{\mathbf{X}}\right)$.

Lemma 2.16. (1) At any point $x \in \mathbf{X}$, the Lie algebra of local Killing fields of $\mathcal{L}_{\mathbf{X}}$ at $x$ is identified with $\mathfrak{s l}_{3}$ through the fundamental vector fields of the action. In other words, the application $v \in \mathfrak{s l}_{3} \mapsto\left[v^{\dagger}\right]_{x} \in \mathfrak{k i l l}_{\mathcal{L}_{\mathbf{X}}}^{\text {loc }}(x)$ sending $v \in \mathfrak{s l}_{3}$ to the germ of $v^{\dagger}$ at $x$, is an anti-isomorphism of Lie algebras.

(2) Any local Killing field of $\left(\mathbf{X}, \mathcal{L}_{\mathbf{X}}\right)$ defined on a connected neighbourhood of a point $x \in \mathbf{X}$ is the restriction of a global Killing field defined on $\mathbf{X}$. In other words, $X \in \mathfrak{K i l l}\left(\mathbf{X}, \mathcal{L}_{\mathbf{X}}\right) \mapsto[X]_{x} \in \mathfrak{k i l l}_{\mathcal{L}_{\mathbf{X}}}^{\text {loc }}(x)$ is a Lie algebra isomorphism.

Proof. 1. If $v^{\dagger}$ is trivial in the neighbourhood of $x$, then for any $t \in \mathbb{R}, \mathrm{e}^{t v}$ acts trivially on an open neighbourhood of $x$. But the action of $\mathbf{G}$ on $\mathbf{X}$ is analytic: if $g$ and $h$ in $\mathbf{G}$ have the same action on some non-empty open subset of $\mathbf{X}$, then $g=h$ (because the linear subspace generated by the pre-image in $\mathbb{R}^{3}$ of a non-empty open subset of $\mathbb{R} \mathbf{P}^{2}$ is equal to $\mathbb{R}^{3}$ ). Therefore, $\mathrm{e}^{t v}=\mathrm{id}$ for any $t \in \mathbb{R}$ and $v=0$. The application $v \mapsto\left[v^{\dagger}\right]_{x}$ is thus injective, and as $\operatorname{dim} \mathfrak{k i r l}_{\mathcal{L}_{\mathbf{X}}}^{l o c}(x) \leq \operatorname{dim} \mathfrak{s l}_{3}$ according to the third assertion of Lemma 2.14, it is an isomorphism. Finally, $v \mapsto v^{\dagger}$ is known to be an anti-morphism of Lie algebras.

2. Any local Killing field at $x$ is the restriction of $v^{\dagger}$ for some $v \in \mathfrak{s l}_{3}$ according to the first assertion, and extends therefore to a Killing field defined on $\mathbf{X}$.

2.4.2. Total curvature map of an enhanced Lagrangian contact structure. Let $(\mathcal{C}, \varphi)=$ $(\hat{M}, \omega, \varphi)$ be the normal Cartan geometry of a three-dimensional enhanced Lagrangian contact structure $(M, \mathcal{S})$. With $K: \hat{M} \rightarrow W_{K}$ the curvature map of $\mathcal{C}$, we define the curvature map

$$
\mathcal{K}:=(K, \varphi): \hat{M} \rightarrow W_{\mathcal{K}}:=W_{K} \times \mathbb{V},
$$

of the enhanced Lagrangian contact structure $(M, \mathcal{S})$, which is $\mathbf{P}_{\text {min }}$-equivariant for the right diagonal action of $\mathbf{P}_{\text {min }}$ on $W_{\mathcal{K}}$.

If $W$ is any manifold endowed with a right action of $\mathbf{P}_{\text {min }}$, we define $B(W):=\{(w, l) \mid$ $\left.w \in W, l \in \mathrm{L}\left(\mathfrak{s l}_{3}, \mathrm{~T}_{w} W\right)\right\}$ (this is a vector bundle over $W$ ), that we endow with the right $\mathbf{P}_{\text {min }}$-action $(w, l) \cdot p=\left(w \cdot p, \mathrm{D}_{w} R_{p} \circ l \circ \operatorname{Ad}(p)\right)$. For any smooth $\mathbf{P}_{\text {min }}$-equivariant map $\psi: \hat{M} \rightarrow W$, we define a $\mathbf{P}_{\text {min }}$-equivariant map $D^{1} \psi: \hat{M} \rightarrow B(W)$ encoding the differential of $\psi$ as follows: $D^{1} \psi(\hat{x})=\left(\psi(\hat{x}), \mathrm{D}_{\hat{x}} \psi \circ \omega_{\hat{x}}^{-1}\right)$. We also define inductively 
$B^{k+1}(W)=B\left(B^{k}(W)\right)$ and $D^{k+1} \psi=D\left(D^{k} \psi\right): \hat{M} \rightarrow B^{k+1}(W)$ for any $k \in \mathbb{N}$ (with $B^{0}(W)=W$ and $\left.D^{0} \phi=\phi\right)$.

Denoting $m=\operatorname{dim} \mathfrak{s l}_{3}=8$, we define $W_{\mathcal{K}^{t o t}}:=B^{m}\left(W_{\mathcal{K}}\right)$, and the total curvature

$$
\mathcal{K}^{t o t}:=D^{m} \mathcal{K}: \hat{M} \rightarrow W_{\mathcal{K}^{t o t}}
$$

of the enhanced Lagrangian contact structure $\mathcal{S}$. The total curvature $\mathcal{K}^{\text {tot }}$ is $\mathbf{P}_{\text {min }}{ }^{-}$ equivariant and preserved by local automorphisms of $\mathcal{S}$ (i.e. for any such local automorphism $f$ we have $\left.\mathcal{K}^{\text {tot }} \circ \hat{f}=\mathcal{K}^{\text {tot }}\right)$. We also define for $k \in \mathbb{N}^{*}$ the space of Killing generators of order $k$ by $\operatorname{Kill}^{k}(\hat{x})=\omega_{\hat{x}}\left(\operatorname{Ker}\left(\mathrm{D}_{\hat{x}} D^{k-1} \mathcal{K}\right)\right) \subset \mathfrak{s l}_{3}$, and the space of Killing generators of total order by $\operatorname{Kill}^{t o t}(\hat{x})=\operatorname{Kill}^{m+1}(\hat{x})=\omega_{\hat{x}}\left(\operatorname{Ker}\left(\mathrm{D}_{\hat{x}} \mathcal{K}^{t o t}\right)\right) \subset \mathfrak{s l}_{3}$.

2.4.3. Gromov's theory. The integrability locus of $\hat{M}$ is defined as the set $\hat{M}^{\text {int }}$ of those points $\hat{x} \in \hat{M}$ such that for any $v \in \operatorname{Kill}^{t o t}(\hat{x})$, there exists a local Killing field $X$ of $\mathcal{S}$ defined around $\pi(\hat{x})$ and such that $\omega_{\hat{x}}\left(\hat{X}_{\hat{x}}\right)=v$. It is easy to check that $\hat{M}^{\text {int }}$ is a

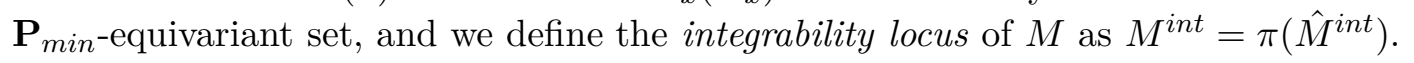

Theorem 2.17 (Integrability theorem). Let $(M, \mathcal{S})$ be a three-dimensional enhanced Lagrangian contact structure of total curvature $\mathcal{K}^{\text {tot }}$, and $\hat{M}$ be its normal Cartan bundle. Then the integrability locus $\hat{M}^{\text {int }}$ of $\hat{M}$ is equal to the set of points $\hat{x} \in \hat{M}$ where the rank of $\mathrm{D}_{\hat{x}} \mathcal{K}^{\text {tot }}$ is locally constant. In particular, $\hat{M}^{\text {int }}$ is open and dense, and so is the integrability locus $M^{\text {int }}$ of $M$.

Gromov investigates in [Gro88] the integration of "jets" of Killing fields for very general rigid geometric structures, and proves results related to the above Theorem. In the case of three-dimensional enhanced Lagrangian contact structures, the equivalence with normal generalized Cartan geometries allows to avoid the notion of jets of Killing fields, replaced by the one of Killing generators of total order. In this setting, Theorem 2.17 is a consequence of [Pec16, Theorem 4.19]. We use here a modification of the statement of Pecastaing proved by Frances in [Fra16, Theorem 2.2]. The proof of the statement of Frances for generalized Cartan geometries is straightforward by following the lines of the proof he does for Cartan geometries, and using [Pec16, Lemma 4.20 and Lemma 4.9].

\section{Quasi homogeneity and Flatness of the StRucture}

From now on and until Paragraph 8.2, we are under the hypotheses of Theorem B and we adopt its notations. $M$ is thus a three-dimensional compact connected and orientable manifold, $\mathcal{S}=\left(E^{\alpha}, E^{\beta}, E^{c}\right)$ is an enhanced Lagrangian contact structure on $M$, and we denote by $\mathcal{L}=\left(E^{\alpha}, E^{\beta}\right)$ its underlying Lagrangian contact structure. Finally, $f$ is an orientation-preserving automorphism of $(M, \mathcal{S})$ such that:

- each of the distributions $E^{\alpha}$ and $E^{\beta}$ is weakly contracted by $f$ (see Definition $1.3)$,

- and $f$ has a dense orbit.

In particular, the non-wandering set $N W(f)=N W\left(f^{-1}\right)$ equals $M$. We recall that in this case, the set $\operatorname{Rec}(f)$ (respectively $\operatorname{Rec}\left(f^{-1}\right)$ ) of recurrent points of $f$ (respectively $\left.f^{-1}\right)$ is a dense $G_{\delta}$-subset of $M$. Therefore, $\operatorname{Rec}(f) \cap \operatorname{Rec}\left(f^{-1}\right)$ is dense in $M$ as well. 
3.1. Quasi homogeneity of the enhanced Lagrangian contact structure. At a point $x \in M$, we introduce the subalgebra

$$
\mathfrak{i s}_{\mathcal{S}}^{l o c}(x)=\left\{X \in \mathfrak{k i r l}_{\mathcal{S}}^{l o c}(x) \mid X(x)=0\right\}
$$

of local Killing fields vanishing at $x$, that we call the isotropy subalgebra of $\mathcal{S}$.

Definition 3.1. The Kill ${ }^{l o c}$-orbit (for $\mathcal{S}$, respectively $\mathcal{L}$ ) of a point $x \in M$ is the set of points that can be reached from $x$ by flowing along finitely many local Killing fields of $\mathcal{S}$ (respectively $\mathcal{L}$ ). An enhanced Lagrangian contact structure $(M, \mathcal{S})$ (resp. a Lagrangian contact structure $(M, \mathcal{L}))$ is locally homogeneous if any connected component of $M$ is a Kill ${ }^{l o c}$-orbit.

The first claim of the following Proposition is a consequence of Gromov's "opendense orbit theorem", and the second one is a reformulation in the context of enhanced Lagrangian contact structures of a work done by Frances in [Fra16, Proposition 5.1] for pseudo-Riemannian structures.

Proposition 3.2. There exists an open and dense subset $\Omega$ of $M$, such that the enhanced Lagrangian contact structure $\mathcal{S}$ is locally homogeneous in restriction to $\Omega$. Moreover for any $x \in \Omega$, the isotropy subalgebra $i_{\mathcal{S}}^{l o c}(x)$ is non-trivial.

Proof. Since $\mathcal{S}$ has an automorphism $f$ with a dense orbit, Gromov's dense orbit theorem directly implies the first claim (see [Gro88, Corollary 3.3.A], and [Pec16, Theorem 4.13] for a proof in the case of generalized Cartan geometries). Since the integrability locus $M^{\text {int }}$ is open and dense (see Theorem 2.17), and $\operatorname{Rec}(f) \cap \operatorname{Rec}\left(f^{-1}\right)$ is dense in $M$, there finally exists a point $x \in \Omega \cap M^{\text {int }} \cap \operatorname{Rec}(f) \cap \operatorname{Rec}\left(f^{-1}\right)$. We show now that $\mathfrak{i s}_{\mathcal{S}}^{\text {loc }}(x)$ is non-zero.

Let us denote by $(\hat{M}, \omega, \varphi)$ the normal generalized Cartan geometry of $\mathcal{S}$ (see Definition 2.13), and choose a lift $\hat{x} \in \pi^{-1}(x)$ in the Cartan bundle. Possibly replacing $f$ by $f^{-1}$, we have $\lim _{n \rightarrow+\infty}\left\|\left.\mathrm{D}_{x} f^{n}\right|_{E^{\alpha}}\right\|=0$, and by hypothesis on $x$, there exists a strictly increasing sequence $n_{k}$ of integers such that $f^{n_{k}}(x)$ converges to $x$, implying the existence of a sequence $p_{k} \in \mathbf{P}_{\text {min }}$ such that $\hat{f}^{n_{k}}(\hat{x}) \cdot p_{k}^{-1}$ converges to $\hat{x}$. We claim that the sequence $\hat{f}^{n_{k}}(\hat{x})$ has to leave every compact subset of $\hat{M}$, implying that $p_{k}$ also leaves every compact subset of $\mathbf{P}_{\text {min }}$. In fact, if not, some subsequence $\left(\hat{f}^{n_{k}^{\prime}}(\hat{x})\right)$ converges in $\hat{M}$, implying that $\left(\hat{f}_{k}^{\prime}\right)$ converges to some diffeomorphism of $\hat{M}$ for the $\mathcal{C}^{\infty}$-topology, because $\hat{f}$ preserves the parallelism defined by $\omega$ (see [Kob95, Theorem I.3.2]). Therefore, $\left(f_{k}^{n_{k}^{\prime}}\right)$ also converges for the $\mathcal{C}^{\infty}$-topology to some diffeomorphism of $M$, contradicting $\lim _{k \rightarrow+\infty}\left\|\left.\mathrm{D}_{x} f^{n_{k}^{\prime}}\right|_{E^{\alpha}}\right\|=0$.

The sequel of the proof of [Fra16, Proposition 5.1] will enable us to conclude, using the total curvature $\mathcal{K}^{t o t}: \hat{M} \rightarrow W_{\mathcal{K}^{t o t}}$ of the generalized Cartan geometry associated to $\mathcal{S}$ (see Paragraph 2.4.3). By $\mathbf{P}_{m i n}$-equivariance of the total curvature and its invariance by automorphisms, $p_{k} \cdot \mathcal{K}^{\text {tot }}(\hat{x})=\mathcal{K}^{\text {tot }}\left(\hat{f}^{n_{k}}(\hat{x}) \cdot p_{k}^{-1}\right)$ converges to $\mathcal{K}^{\text {tot }}(\hat{x})$. The manifold $W_{\mathcal{K}^{\text {tot }}}$ has a canonical structure of algebraic variety for which the action of $\mathbf{P}_{\min }$ is algebraic (because its action on the space $W_{K}$ of normal curvatures and on the algebraic variety $\mathbb{V} \subset \mathbf{P}\left(\mathfrak{s l}_{3} / \mathfrak{p}_{\text {min }}\right)$ are algebraic, see [Pec16, Remark 4.16] for more details). Therefore, the orbits of the action of $\mathbf{P}_{\min }$ on $W_{\mathcal{K}^{t o t}}$ are locally closed, and are thus imbedded 
submanifolds. In particular, there exists a sequence $\varepsilon_{k} \in \mathbf{P}_{\min }$ converging to the identity and such that $p_{k} \cdot \mathcal{K}^{\text {tot }}(\hat{x})=\varepsilon_{k} \cdot \mathcal{K}^{\text {tot }}(\hat{x})$, i.e. such that $\varepsilon_{k}^{-1} p_{k} \in \operatorname{Stab}_{\mathbf{P}_{\text {min }}}\left(\mathcal{K}^{\text {tot }}(\hat{x})\right)$. As $\varepsilon_{k}^{-1} p_{k}$ leaves every compact subset of $\mathbf{P}_{\text {min }}, \operatorname{Stab}_{\mathbf{P}_{\text {min }}}\left(\mathcal{K}^{t o t}(\hat{x})\right)<\mathbf{P}_{\text {min }}$ is non-compact. But $\operatorname{Stab}_{\mathbf{P}_{\text {min }}}\left(\mathcal{K}^{\text {tot }}(\hat{x})\right)$ is an algebraic subgroup of $\mathbf{P}_{\text {min }}$ and has thus a finite number of connected components, finally implying that its identity component is also non-compact.

There exists thus a non-zero vector $v \in \mathfrak{p}_{\text {min }}$ in the Lie algebra of $\operatorname{Stab}_{\mathbf{P}_{\text {min }}}\left(\mathcal{K}^{\text {tot }}(\hat{x})\right)$. For any $t \in \mathbb{R}$ we have by hypothesis $\mathcal{K}^{\text {tot }}(\hat{x} \cdot \exp (t v))=\mathcal{K}^{\text {tot }}(\hat{x}) \cdot \exp (t v)=\mathcal{K}^{t o t}(\hat{x})$, and deriving this equality at $t=0$ we obtain $\mathrm{D}_{\hat{x}} \mathcal{K}^{t o t}\left(\omega_{\hat{x}}^{-1}(v)\right)=0$, i.e. $v \in \omega_{\hat{x}}\left(\operatorname{Ker}\left(\mathrm{D}_{\hat{x}} \mathcal{K}^{\text {tot }}\right)\right)=$ $\operatorname{Kill}^{t o t}(\hat{x})$. As $\hat{x}$ is in the integrability locus $\hat{M}^{\text {int }}$ of $\hat{M}$, there exists a local Killing field $X \in \mathfrak{k i r l}_{\mathcal{S}}^{l o c}(x)$ such that $\omega_{\hat{x}}\left(\hat{X}_{\hat{x}}\right)=v \neq 0$, implying in particular that $X \neq 0$ and $X(x)=0$, i.e. that $X \in \mathfrak{i s}_{\mathcal{S}}^{\text {loc }}(x) \backslash\{0\}$.

The isotropy subalgebra at any point $y \in \Omega$ being linearly isomorphic to the one at $x$ because $\Omega$ is an Aut ${ }^{l o c}$-orbit, $\mathfrak{i s}_{\mathcal{S}}^{l o c}(y)$ is finally non-zero at any point $y \in \Omega$, which finishes the proof of the corollary.

3.2. Flatness of the Lagrangian contact structure. In particular, the underlying Lagrangian contact structure $\mathcal{L}=\left(E^{\alpha}, E^{\beta}\right)$ is also locally homogeneous with non-zero isotropy in restriction to the open and dense subset $\Omega$. The following result due to Tresse in [Tre96] (see also [KT17, §4.5.2]) implies that $\left.\mathcal{L}\right|_{\Omega}$ is flat.

Theorem 3.3 (Tresse [Tre96]). Any three-dimensional locally homogeneous connected Lagrangian contact structure with non-zero isotropy is flat.

By density of $\Omega$ and continuity of the curvature, the Lagrangian contact structure $(M, \mathcal{L})$ is therefore flat, and according to Paragraph 2.3.2, we obtain the following.

Corollary 3.4. The Lagrangian contact structure $\mathcal{L}$ is described by a $(\mathbf{G}, \mathbf{X})$-structure on $M$.

The rest of this paragraph is devoted to give a self-contained proof of Tresse's Theorem 3.3. We consider a locally homogeneous Lagrangian contact structure $\mathcal{L}$ with non-zero isotropy defined on a three-dimensional connected manifold $M$. We denote by $(M, \mathcal{C})=$ $(M, \hat{M}, \omega)$ the normal Cartan geometry of $\mathcal{L}$, and by $K: \hat{M} \rightarrow W_{K}$ its curvature map. Choosing $x \in M$ and $\hat{x} \in \hat{M}$, it suffices to prove that $K(\hat{x})=0$ by local homogeneity of $\mathcal{C}$. We will denote by

$$
\mathfrak{h}=\mathfrak{k i f l}_{\mathcal{L}}^{l o c}(x) \text { and } \mathfrak{i}=\mathfrak{i s}_{\mathcal{L}}^{l o c}(x)
$$

the algebra of local Killing fields of $\mathcal{L}$ at $x$ and its isotropy subalgebra. As $\mathcal{L}$ is locally homogeneous, $\operatorname{ev}_{x}(\mathfrak{h}):=\{X(x) \mid X \in \mathfrak{h}\}=\mathrm{T}_{x} M$, and in particular $\operatorname{dim} \mathfrak{h}-\operatorname{dim} \mathfrak{i}=3$. The following result gives us a sufficient condition for the vanishing of the curvature.

Lemma 3.5. Let $f$ be a local automorphism of a locally homogeneous three-dimensional Lagrangian contact structure $(M, \mathcal{L})$ fixing a point $x \in M$, let $\hat{x} \in \pi^{-1}(x)$ be a lift of $x$ in the normal Cartan bundle of $\mathcal{L}$, and let $p \in \mathbf{P}_{\text {min }}$ be the holonomy of $\hat{f}$ at $\hat{x}$, characterized by $\hat{f}(\hat{x})=\hat{x} \cdot p^{-1}$. If $p=\exp (v)$ with

$$
v=\left(\begin{array}{ccc}
a & * & * \\
0 & -a-b & * \\
0 & 0 & b
\end{array}\right) \in \mathfrak{p}_{\text {min }} \text { such that } b \neq-5 a \text { and } a \neq-5 b,
$$


then $\mathcal{L}$ is flat.

Proof. Since the curvature $K$ is preserved by $\hat{f}$ and $\mathbf{P}_{\text {min }}$-equivariant (see paragraph $2.3)$, we obtain $p \cdot K(\hat{x})=K\left(\hat{x} \cdot p^{-1}\right)=K(\hat{f}(\hat{x}))=K(\hat{x})$, where the holonomy $p$ is of the form

$$
p=\left(\begin{array}{ccc}
\lambda & * & * \\
0 & \lambda^{-1} \mu^{-1} & * \\
0 & 0 & \mu
\end{array}\right)
$$

with $\mu \neq \lambda^{-5}$ and $\lambda \neq \mu^{-5}$ by hypothesis. According to the expression of the components $(p . K)_{\alpha}$ and $(p . K)_{\beta}$ of the curvature given in (2.11), we have $\lambda \mu^{5} K(\hat{x})_{\alpha}=K(\hat{x})_{\alpha}$ and $\lambda^{5} \mu K(\hat{x})_{\beta}=K(\hat{x})_{\beta}$, implying $K(\hat{x})_{\alpha}=K(\hat{x})_{\beta}=0$. The structure being locally homogeneous and the subspace $W_{H}=\left\{K \in W_{K} \mid K_{\alpha}=K_{\beta}=0\right\}$ being $\mathbf{P}_{\text {min }}$-invariant, $K$ has values in $W_{H}$ on a neighbourhood of $\hat{x}$, and therefore $K=0$ on this neighbourhood according to Proposition 2.11. By local homogeneity, $\mathcal{L}$ is flat.

We introduce the Cartan subalgebra $\mathfrak{a} \simeq \mathbb{R}^{2}$ of diagonal matrices of $\mathfrak{p}_{\text {min }}$, and the projection $p: \mathfrak{p}_{\text {min }} \rightarrow \mathfrak{a}$ on $\mathfrak{a}$ parallel to $\mathfrak{h e i s}(3)$, which is a Lie algebra morphism. The following linear map will play an important role in the proof:

$$
\phi: X \in \mathfrak{i} \mapsto p\left(\omega_{\hat{x}}\left(\hat{X}_{\hat{x}}\right)\right) \in \mathfrak{a} .
$$

Fact 3.6. If there exists $X \in \mathfrak{i}$ such that $\phi(X)$ satisfies the hypotheses (3.2) of Lemma 3.5, then $\mathcal{L}$ is flat.

Proof. We have the following relation for any $t \in \mathbb{R}$

$$
\varphi_{\hat{X}}^{t}(\hat{x})=\hat{x} \cdot \exp \left(t \omega_{\hat{x}}\left(\hat{X}_{\hat{x}}\right)\right)
$$

Denoting by $p(t)$ the element of $\mathbf{P}_{\min }$ such that $\varphi_{\hat{X}}^{t}(\hat{x})=\hat{x} \cdot p(t),\{p(t)\}_{t \in \mathbb{R}}$ is a oneparameter subgroup. There exists thus $w \in \mathfrak{p}_{\min }$ such that $p(t)=\exp (t w)$, and deriving the relation $\varphi_{\hat{X}}^{t}(\hat{x})=\hat{x} \cdot \exp (t w)$ at $t=0$ we obtain $\omega_{\hat{x}}\left(\hat{X}_{\hat{x}}\right)=w$ (because the Cartan connection $\omega$ reproduces the fundamental vector fields of the action of $\mathbf{P}_{\min }$ ). There exists thus an automorphism $\varphi$ of $(M, \mathcal{L})$ fixing $x$ and such that $\hat{\varphi}(\hat{x})=\hat{x} \cdot \exp \left(\omega_{\hat{x}}\left(\hat{X}_{\hat{x}}\right)\right)^{-1}$. As $\phi(X)=p\left(\omega_{\hat{x}}\left(\hat{X}_{\hat{x}}\right)\right)$ satisfies the conditions $(3.2), \omega_{\hat{x}}\left(\hat{X}_{\hat{x}}\right)$ also does, and Lemma 3.5 implies that $\mathcal{L}$ is flat.

Fact. If $\operatorname{Ker}(\phi) \neq\{0\}$ then $\mathcal{L}$ is flat.

Proof. There exists then $X \in \mathfrak{i}$ such that $v:=\omega_{\hat{x}}\left(\hat{X}_{\hat{x}}\right) \in \mathfrak{h} \mathfrak{e i s}(3)=\left(\mathfrak{s l}_{3}\right)^{1}$, i.e.

$$
v=\left(\begin{array}{lll}
0 & a & c \\
0 & 0 & b \\
0 & 0 & 0
\end{array}\right) \neq 0 .
$$

We first assume that $(a, b) \neq(0,0)$. For an element of the form $w=\left(\begin{array}{ccc}0 & 0 & 0 \\ a^{\prime} & 0 & 0 \\ 0 & b^{\prime} & 0\end{array}\right)$ in $\mathfrak{s l}_{3}$, we have $[v, w]=\left(\begin{array}{ccc}a a^{\prime} & * & * \\ 0 & b b^{\prime}-a a^{\prime} & * \\ 0 & 0 & -b b^{\prime}\end{array}\right)$, and as $a \neq 0$ or $b \neq 0$, there exists such an element $w \in \mathfrak{s l}_{3}$ satisfying $[v, w]=\left(\begin{array}{ccc}1 & * & 0 \\ 0 & -1 & * \\ 0 & 0 & 0\end{array}\right)$ or $[v, w]=\left(\begin{array}{ccc}0 & * & 0 \\ 0 & 1 & * \\ 0 & 0 & -1\end{array}\right)$. As $\mathcal{L}$ is locally homogeneous, there exists a Killing field $Y \in \mathfrak{h}$ such that $Y_{o}=\mathrm{D}_{\hat{x}} \pi\left(\omega_{\hat{x}}^{-1}(w)\right)$, implying $\omega_{\hat{x}}\left(\hat{Y}_{\hat{x}}\right)=w+w_{0}$ with $w_{0} \in \mathfrak{p}_{\text {min }}=\left(\mathfrak{s l}_{3}\right)^{0}$. We now use the relation

$$
\omega([\hat{X}, \hat{Y}])=-[\omega(\hat{X}), \omega(\hat{Y})]+K(\omega(\hat{X}), \omega(\hat{Y})) .
$$


verified for any Killing fields of the Cartan geometry $\mathcal{C}$, that will be proved at the end of this demonstration. We obtain $\omega_{\hat{x}}\left([\hat{X}, \hat{Y}]_{\hat{x}}\right)=-[v, w]+\left[v, w_{0}\right]+K\left(v, w+w_{0}\right) \in \mathfrak{p}_{\text {min }}$, where $\left[v, w_{0}\right] \in\left(\mathfrak{s l}_{3}\right)^{1}$ according to the filtration property of $\mathfrak{s l}_{3}$, and $K\left(v, w+w_{0}\right)=0$ because $v \in \mathfrak{p}_{\text {min }}$ (see Paragraph 2.3). In particular $[X, Y] \in \mathfrak{i}$, and $\phi([X, Y])$ is equal to one of the diagonal matrices $[1,-1,0]$ or $[0,1,-1]$, that both satisfy the condition (3.2). Therefore $\mathcal{L}$ is flat according to Fact 3.6.

If $a=b=0$, we can find an element $w \in \mathfrak{s l}_{3}$ such that $[v, w]=\left(\begin{array}{ccc}1 & 0 & 0 \\ 0 & 0 & 0 \\ 0 & 0 & -1\end{array}\right)$, and by the same argument as above we find $Y \in \mathfrak{h}$ such that $[X, Y] \in \mathfrak{i}$ and $\phi([X, Y])=\left(\begin{array}{ccc}1 & 0 & 0 \\ 0 & 0 & 0 \\ 0 & 0 & -1\end{array}\right)$. As this element of $\mathfrak{a}$ satisfies the conditions $(3.2), \mathcal{L}$ is flat by Fact 3.6.

We now prove the relation (3.4) for two Killing fields $X$ and $Y$ of the Cartan geometry $\mathcal{C}$. Since the flow of $X$ preserves $\omega$, the Lie derivative $L_{X} \omega$ vanishes identically, and applying Cartan's formula $L_{X}=d \circ \iota_{X}+\iota_{X} \circ d$ to $Y$, we obtain $Y \cdot \omega(X)+d \omega(X, Y)=0$. Cartan's formula $d \omega(X, Y)=X \cdot \omega(Y)-Y \cdot \omega(X)-\omega([X, Y])$ implies $X \cdot \omega(Y)=\omega([X, Y])$, and as $L_{Y} \omega=0$ as well, we also have $-Y \cdot \omega(X)=\omega([X, Y])$. Equation (3.4) then follows from the definition of the curvature.

Fact. If $\phi(\mathfrak{i})=\mathfrak{a}$ then $\mathcal{L}$ is flat.

Proof. There exists in this case a Killing field $X \in \mathfrak{i}$ such that $\phi(X)=\left(\begin{array}{ccc}1 & 0 & 0 \\ 0 & 0 & 0 \\ 0 & 0 & -1\end{array}\right)$, which satisfies the hypotheses (3.2), implying that $\mathcal{L}$ is flat according to Fact 3.6.

It remains to handle the case when $\phi$ is injective, and $\phi(\mathfrak{i})$ is one-dimensional. There exists then $V \in \mathfrak{i}$ such that $\mathfrak{i}=\mathbb{R} V$, and we can moreover assume without lost of generality that $v:=\omega_{\hat{x}}\left(\hat{V}_{\hat{x}}\right) \in \mathfrak{p}_{\text {min }}$ does not verify the condition (3.2) (if it does, then $\mathcal{L}$ is flat according to Fact 3.6). In other words, denoting the components of $v$ in $\mathfrak{a}$ by

$$
\phi(V)=p(v)=\left(\begin{array}{ccc}
a & 0 & 0 \\
0 & -a-b & 0 \\
0 & 0 & b
\end{array}\right) \in \mathfrak{a}
$$

with $(a, b) \in \mathbb{R}^{2}$, we assume that

$$
\text { either } a=-5 b \neq 0 \text {, or } b=-5 a \neq 0 .
$$

Since $v \in \mathfrak{p}_{\text {min }}$, the curvature part of the relation (3.4) vanishes, and for any $X \in \mathfrak{h}$ we have:

$$
\left.\omega_{\hat{x}}(\widehat{[V, X]}]_{\hat{x}}\right)=-\left[v, \omega_{\hat{x}}\left(\hat{X}_{\hat{x}}\right)\right] .
$$

The linear map

$$
\varphi: X \in \mathfrak{h} \mapsto \omega_{\hat{x}}\left(\hat{X}_{\hat{x}}\right) \in \mathfrak{s l}_{3}
$$

is injective according to Lemma 2.14, and as $\operatorname{ev}_{x}(\mathfrak{h})=\mathrm{T}_{x} M$ by local homogeneity, $\varphi$ induces an isomorphism $\bar{\varphi}$ between $\mathfrak{h} / \mathfrak{i}$ and $\mathfrak{s l}_{3} / \mathfrak{p}_{\text {min }}$. Using the notations (2.8) in Paragraph 2.2.3 for the basis $\left(\bar{e}_{\alpha}, \bar{e}_{\beta}, \bar{e}_{0}\right)$ of $\mathfrak{s l}_{3} / \mathfrak{p}_{\text {min }}$, there exists $X, Y$, and $Z$ in $\mathfrak{h}$ such that $\varphi(X) \in e_{\alpha}+\mathfrak{p}_{\text {min }}, \varphi(Y) \in e_{\beta}+\mathfrak{p}_{\text {min }}$, and $\varphi(Z) \in e_{0}+\mathfrak{p}_{\text {min }}$. According to (3.6), $\bar{\varphi}$ intertwines the adjoint action of $V$ on $\mathfrak{h} / \mathfrak{i}$ and the adjoint action of $-v$ on $\mathfrak{s l}_{3} / \mathfrak{p}_{\text {min }}$, 
implying

$$
\operatorname{Mat}_{(\bar{X}, \bar{Y}, \bar{Z})}(\overline{\operatorname{ad}}(V))=\operatorname{Mat}_{\left(\bar{e}_{\alpha}, \bar{e}_{\beta}, \bar{e}_{0}\right)}(\overline{\operatorname{ad}}(-v))=\left(\begin{array}{ccc}
-a-2 b & 0 & * \\
0 & 2 a+b & * \\
0 & 0 & a-b
\end{array}\right) .
$$

We will denote by $A=-a-2 b$ and $B=2 a+b$ the eigenvalues of $\overline{a d}(V)$ with respect to $\bar{X}$ and $\bar{Y}$. Our hypotheses (3.5) on $a$ and $b$ imply $A \neq 0$ and $B \neq 0$, allowing us to choose $X$ and $Y$ in $\mathfrak{h}$ satisfying

$$
[V, X]=A X \text { and }[V, Y]=B Y .
$$

In fact, if $X \in \mathfrak{h}$ satisfies $\bar{\varphi}(X+\mathfrak{i})=\bar{e}_{\alpha}$, there exists $\lambda \in \mathbb{R}$ such that $[V, X]=a X+\lambda V$ according to (3.7), and $X^{\prime}=X+\frac{\lambda}{A} V$ satisfies then $\left[V, X^{\prime}\right]=A X^{\prime}$. We deal with the case of $Y$ by the same computations.

The Jacobi identity yields $[V,[X, Y]]=(A+B)[X, Y]$, implying in particular that $[X, Y] \notin \operatorname{Vect}(X, Y, V)$ since $A+B$ is distinct from $A, B$ and 0 . A second application of the same identity gives $[V,[X,[X, Y]]]=(2 A+B)[X,[X, Y]]$ and $[V,[Y,[X, Y]]]=$ $(A+2 B)[Y,[X, Y]]$. Furthermore, if $[X,[X, Y]] \neq 0$, then $2 A+B$ is an eigenvalue of $\operatorname{ad}(V) \in \mathrm{L}(\mathfrak{h})$, and is thus equal to one of the eigenvalues $A, B$, or $A+B$ (since $\operatorname{dim} \mathfrak{h}=4$ and $2 A+B \neq 0$ ). But the equalities $2 A+B=A+B$ or $2 A+B=B$ would contradict $A \neq 0$, and the equality $2 A+B=A$ would likewise contradict our hypotheses on $a$ and $b$. Consequently, $[X,[X, Y]]=0$, and for the same reasons $[Y,[X, Y]]=0$.

As a consequence, $\mathcal{E}:=\operatorname{Vect}(X, Y,[X, Y])$ is a subalgebra of $\mathfrak{h}$ isomorphic to $\mathfrak{h e i s}(3)$. There is a connected open neighbourhood $U$ of $x$ such that the injective linear map $X \in \mathfrak{K i l l}(U, \mathcal{L}) \mapsto[X]_{x} \in \mathfrak{k i r l}_{\mathcal{L}}^{\text {loc }}(x)$ is an isomorphism (see Remark 2.15), and there is thus an injective Lie algebra morphism $\iota: \mathfrak{h e i s}(3) \rightarrow \mathfrak{K i l t}(U, \mathcal{L})$ of image $\mathcal{E}$. According to the work of Palais in [Pal57], chapter II Theorem XI and its corollary, there exists a (unique) local action of Heis(3) on $U$ that integrates this infinitesimal action, i.e. such that $X^{\dagger}=\left.\iota(X)\right|_{U}$ for any $X \in \mathfrak{h e i s}(3)$. In particular, the local action of Heis(3) on $U$ preserves $\mathcal{L}$, and as $\iota(\mathfrak{h e i s}(3)) \cap \mathfrak{i}=\{0\}$, the orbital map at $x$ is a Heis(3)-equivariant embedding. The Lagrangian contact structure $\mathcal{L}$ is thus locally isomorphic to a leftinvariant Lagrangian contact structure on Heis(3). The following lemma implies then that $\mathcal{L}$ is flat, finishing the proof of Theorem 3.3.

Lemma 3.7. Any left-invariant Lagrangian contact structure on Heis(3) is flat.

Proof. The left-invariant Lagrangian contact structure $\mathcal{M}_{0}=(\mathbb{R} \tilde{X}, \mathbb{R} \tilde{Y})$ of Heis(3) generated by $X=\left(\begin{array}{lll}0 & 1 & 0 \\ 0 & 0 & 0 \\ 0 & 0 & 0\end{array}\right)$ and $Y=\left(\begin{array}{lll}0 & 0 & 0 \\ 0 & 0 & 1 \\ 0 & 0 & 0\end{array}\right)$ is flat. In fact, we will see in Paragraph 4.2 .3 that (Heis $(3), \mathcal{M}_{0}$ ) is isomorphic to an open subset of the homogeneous model space $\left(\mathbf{X}, \mathcal{L}_{\mathbf{X}}\right)$. Considering a left-invariant Lagrangian contact structure $\mathcal{M}$ on Heis(3), it suffices thus to find an isomorphism of Lagrangian contact structures from $\mathcal{M}_{0}$ to $\mathcal{M}$ to prove our claim.

There exists $v, w \in \mathfrak{h e i s}(3)$ such that $\mathcal{M}=(\mathbb{R} \tilde{v}, \mathbb{R} \tilde{w})$, and as $\mathbb{R} \tilde{v} \oplus \mathbb{R} \tilde{w}$ is a contact distribution, $[v, w] \notin \operatorname{Vect}(v, w)$. Denoting $Z=\left(\begin{array}{lll}0 & 0 & 1 \\ 0 & 0 & 0 \\ 0 & 0 & 0\end{array}\right), v=a X+b Y+c Z$, and $w=a^{\prime} X+b^{\prime} Y+c^{\prime} Z$, we have $[v, w]=\left(a b^{\prime}-b a^{\prime}\right) Z$, which implies $a b^{\prime}-b a^{\prime} \neq 0$. The Lie algebra automorphism $\varphi$ of $\mathfrak{h e i s}(3)$ whose matrix in the basis $(X, Y, Z)$ is $\left(\begin{array}{lll}a & a \prime^{\prime} & 0 \\ b & b^{\prime} & 0 \\ c & c^{\prime} & a b^{\prime}-b a^{\prime}\end{array}\right)$ 
sends $(X, Y)$ to $(v, w)$, and as Heis(3) is simply-connected, there exists a Lie group automorphism $\phi$ of Heis(3) whose differential at identity is $\varphi$. Since $\phi$ is an automorphism, $\mathrm{D}_{e} \phi(X, Y)=(v, w)$ implies $\phi^{*} \tilde{v}=\tilde{X}$ and $\phi^{*} \tilde{w}=\tilde{Y}$, i.e. $\phi$ is an isomorphism of Lagrangian contact structures from $\mathcal{M}_{0}$ to $\mathcal{M}$.

\section{Local model of the EnHANCED Lagrangian CONTACT STRUCTURe}

In the previous section, we proved that the Lagrangian contact structure $\mathcal{L}$ is locally isomorphic to the homogeneous model space $\left(\mathbf{X}, \mathcal{L}_{\mathbf{X}}\right)$, and thus described by a $(\mathbf{G}, \mathbf{X})$ structure on $M$. The classical strategy is then to reduce the possibilities for the images of the developping map $\delta: \tilde{M} \rightarrow M$ and of the holonomy morphism $\rho: \pi_{1}(M) \rightarrow \mathbf{G}$ of this structure.

In the case studied by Ghys in [Ghy87] of an Anosov flow preserving the structure, the holonomy group $\rho\left(\pi_{1}(M)\right) \subset \mathbf{G}$ is centralized by a one-parameter subgroup of $\mathbf{G}$, which reduce dramatically the possibilities for $\rho\left(\pi_{1}(M)\right)$. But in the case of a discrete-time dynamics, we do not have any relevant algebraic restriction of this kind on $\rho\left(\pi_{1}(M)\right)$.

For this reason, we have to look not only at the local homogeneity of $\mathcal{L}$ on $\Omega$, but at the local homogeneity of the whole enhanced Lagrangian contact structure $\mathcal{S}=\left(E^{\alpha}, E^{\beta}, E^{c}\right)$ on this open dense subset. In this section, we will show that in restriction to $\Omega, \mathcal{S}$ is locally isomorphic to an infinitesimal homogeneous model, that preserves a distribution transverse to the contact plane.

4.1. Two algebraic models. We begin by describing this models in an algebraic way.

4.1.1. Left-invariant structure on $\mathrm{SL}_{2}(\mathbb{R})$. We will use the following basis for the Lie algebra $\mathfrak{s l}_{2}$ of $\mathrm{SL}_{2}(\mathbb{R})$ :

$$
E=\left(\begin{array}{ll}
0 & 1 \\
0 & 0
\end{array}\right), F=\left(\begin{array}{ll}
0 & 0 \\
1 & 0
\end{array}\right) \text {, and } H=\left(\begin{array}{cc}
1 & 0 \\
0 & -1
\end{array}\right) .
$$

The Lie bracket relation $[E, F]=H$ between these three vectors shows that they define a left-invariant enhanced Lagrangian contact structure $\mathcal{S}_{\mathrm{SL}_{2}(\mathbb{R})}=(\mathbb{R} \tilde{E}, \mathbb{R} \tilde{F}, \mathbb{R} \tilde{H})$ on $\mathrm{SL}_{2}(\mathbb{R})$. Moreover, the right action of the one-parameter subgroup $A$ generated by $H$ preserves $\mathcal{S}_{\mathrm{SL}_{2}(\mathbb{R})}$. We endow the universal cover $\widetilde{\mathrm{SL}}_{2}(\mathbb{R})$ of $\mathrm{SL}_{2}(\mathbb{R})$ with the pullback of $\mathcal{S}_{\mathrm{SL}_{2}(\mathbb{R})}$, so that the right action of the one-parameter subgroup $\tilde{A}$ of $\widetilde{S L}_{2}(\mathbb{R})$ generated by $H$ preserves $\mathcal{S}_{\widetilde{\mathrm{SL}}_{2}(\mathbb{R})}$.

Let $\Gamma_{0}$ be a cocompact lattice of $\widetilde{\mathrm{SL}}_{2}(\mathbb{R})$, and $u: \Gamma_{0} \rightarrow \tilde{A}$ be a morphism whose graphgroup $\Gamma=\left\{(\gamma, u(\gamma)) \mid \gamma \in \Gamma_{0}\right\}$ acts freely, properly, and cocompactly on $\widetilde{\mathrm{SL}}_{2}(\mathbb{R})$, via the action $(g, a) \cdot x=g x a$ (these morphisms are called admissible by Salein and studied in detail in his thesis [Sal99]). Then the standard structure of $\widetilde{\mathrm{SL}}_{2}(\mathbb{R})$ is preserved by $\Gamma$, and $\Gamma \backslash \widetilde{\mathrm{SL}}_{2}(\mathbb{R})$ is endowed with the induced enhanced Lagrangian contact structure $\mathcal{S}$, whose distributions are exactly the invariant distributions of the algebraic contact-Anosov flow $\left(R_{a^{t}}\right)$ on $\Gamma \backslash \widetilde{\mathrm{SL}}_{2}(\mathbb{R})$.

4.1.2. Left-invariant structure on Heis(3). We will use the following basis for the Lie algebra $\mathfrak{h} \mathfrak{e i s}(3)$ of $\operatorname{Heis}(3)$ :

$$
X=\left(\begin{array}{lll}
0 & 1 & 0 \\
0 & 0 & 0 \\
0 & 0 & 0
\end{array}\right), Y=\left(\begin{array}{lll}
0 & 0 & 0 \\
0 & 0 & 1 \\
0 & 0 & 0
\end{array}\right), Z=\left(\begin{array}{lll}
0 & 0 & 1 \\
0 & 0 & 0 \\
0 & 0 & 0
\end{array}\right) .
$$


According to the Lie bracket relation $[X, Y]=Z, \mathcal{S}_{\mathrm{Heis}(3)}=(\mathbb{R} \tilde{X}, \mathbb{R} \tilde{Y}, \mathbb{R} \tilde{Z})$ is a leftinvariant enhanced Lagrangian contact structure on Heis(3). The subgroup

$$
\mathcal{A}=\left\{\varphi_{\lambda, \mu} \mid(\lambda, \mu) \in \mathbb{R}^{* 2}\right\}
$$

of automorphisms introduced in the introduction (see (1.1)) is exactly the subgroup of Aut(Heis(3)) preserving $\mathcal{S}_{\text {Heis(3) }}$.

Any cocompact lattice $\Gamma$ of Heis(3) preserves $\mathcal{S}_{\text {Heis(3) }}$, and the quotient $\Gamma \backslash \operatorname{Heis}(3)$ will always be endowed with the induced enhanced Lagrangian contact structure $\mathcal{S}$. The invariant distributions of a partially hyperbolic affine automorphism $L_{g} \circ \varphi$ of $\Gamma \backslash \operatorname{Heis}(3)$, with $g \in \operatorname{Heis}(3)$ and $\varphi \in \mathcal{A}$, are precisely given by $\mathcal{S}$.

4.2. Two homogeneous open subsets of the model space. The left-invariant structures of $\mathrm{SL}_{2}(\mathbb{R})$ and Heis $(3)$ can be geometrically imbedded in $\mathbf{X}$ as homogeneous open subsets, that will be the local models of the enhanced Lagrangian contact structure $\mathcal{S}$ in restriction to $\Omega$.

4.2.1. Some specific surfaces of $\mathbf{X}$, and one affine chart. For $D$ a projective line of $\mathbb{R} \mathbf{P}^{2}$, we define the $\beta-\alpha$ surface

$$
\mathcal{S}_{\beta, \alpha}(D)=\pi_{\alpha}^{-1}(D)=\cup_{y \in \mathcal{C}^{\beta}(D)} \mathcal{C}^{\alpha}(y)
$$

and for $m \in \mathbb{R} \mathbf{P}^{2}$, the analog $\alpha-\beta$ surface

$$
\mathcal{S}_{\alpha, \beta}(m)=\pi_{\beta}^{-1}\left(\left\{L \in \mathbb{R} \mathbf{P}_{*}^{2} \mid m \in L\right\}\right)=\cup_{y \in \mathcal{C}^{\alpha}(m)} \mathcal{C}^{\beta}(y) .
$$

The open subset

$$
\Omega_{a}:=\mathbf{X} \backslash \mathcal{S}_{\beta, \alpha}\left(\left[e_{1}, e_{2}\right]\right)
$$

of $\mathbf{X}$, composed by pointed projective lines $(m, D)$ for which $m \notin\left[e_{1}, e_{2}\right]$, will be identified with the set $\mathbf{X}_{a}$ of pointed affine lines of $\mathbb{R}^{2}$ as follows:

$$
\phi_{a}:(m, D) \in \Omega_{a} \mapsto(m \cap P, D \cap P) \in \mathbf{X}_{a},
$$

where $\operatorname{Vect}\left(e_{1}, e_{2}\right)+(0,0,1)$ is identified with $\mathbb{R}^{2}$ by translation. The diffeomorphism $\phi_{a}$ is moreover equivariant for the canonical identification

$$
\left[\begin{array}{cc}
A & X \\
0 & 1
\end{array}\right] \in \operatorname{Stab}_{\mathbf{G}}\left(\Omega_{a}\right) \mapsto A+X \in \operatorname{Aff}\left(\mathbb{R}^{2}\right)
$$

of $\operatorname{Stab}_{\mathbf{G}}\left(\Omega_{a}\right)$ with the group of affine transformations of $\mathbb{R}^{2}$.

4.2.2. The open subset $Y_{\mathbf{t}}$. We will embed $\mathrm{SL}_{2}(\mathbb{R})$ in $\mathbf{G}$ as follows:

$$
\iota: g \in \mathrm{SL}_{2}(\mathbb{R}) \mapsto\left[\begin{array}{ll}
g & 0 \\
0 & 1
\end{array}\right] \in \mathbf{G} .
$$

The resulting copy $S_{0}$ of $\mathrm{SL}_{2}(\mathbb{R})$ acts simply transitively at $o_{\mathbf{t}}=\left([1,0,1],\left[(1,0,1), e_{2}\right]\right)=$ $\phi_{a}^{-1}\left(e_{1}+\mathbb{R} e_{2}\right) \in \Omega_{a}$, and its orbit $Y_{\mathbf{t}}=S_{0} \cdot o_{\mathbf{t}}$ can be described as

$$
Y_{\mathbf{t}}=\Omega_{a} \backslash S_{\alpha, \beta}\left[e_{3}\right]=\phi_{a}^{-1}\left(\left\{m+L \mid m \in \mathbb{R}^{2} \backslash\{(0,0)\}, L \in \mathbb{R} \mathbf{P}^{1} \backslash\{\mathbb{R} m\}\right\}\right) .
$$

The left-invariant structure of $\mathrm{SL}_{2}(\mathbb{R})$ induces on $Y_{\mathbf{t}}$ a $S_{0}$-invariant enhanced Lagrangian contact structure

$$
\mathcal{S}_{\mathbf{t}}=\left(\theta_{o_{\mathbf{t}}} \circ \iota\right)_{*} \mathcal{S}_{\mathrm{SL}_{2}(\mathbb{R})},
$$


which is compatible with $\mathcal{L}_{\mathbf{X}}$ in the sense that its $\alpha$ and $\beta$-distributions coincide with the ones of $\mathcal{L}_{\mathbf{X}}$, and whose central distribution is entirely described by its value at $o_{\mathbf{t}}$ :

$$
\mathcal{E}_{\mathbf{t}}^{c}\left(o_{\mathbf{t}}\right)=\mathbb{R} H_{0}^{\dagger}\left(o_{\mathbf{t}}\right) \text {, where } H_{0}=\left(\begin{array}{ccc}
1 & 0 & 0 \\
0 & -1 & 0 \\
0 & 0 & 0
\end{array}\right) \text {. }
$$

We denote by $A^{ \pm}$the subgroup of $\mathrm{SL}_{2}(\mathbb{R})$ composed by diagonal matrices. The right action of $A^{ \pm}$preserves $\mathcal{S}_{\mathrm{SL}_{2}(\mathbb{R})}$, and the direct product $\mathrm{SL}_{2}(\mathbb{R}) \times A^{ \pm}$acts on $\mathrm{SL}_{2}(\mathbb{R})$ by $(g, a) \cdot h=g h a$. The isomorphism from $\mathrm{SL}_{2}(\mathbb{R})$ to $\left(Y_{\mathbf{t}}, \mathcal{S}_{\mathbf{t}}\right)$ given by the orbital map at $o_{\mathrm{t}}$ is equivariant for the identification

$$
\left(g,\left(\begin{array}{cc}
\lambda & 0 \\
0 & \lambda^{-1}
\end{array}\right)\right) \in \mathrm{SL}_{2}(\mathbb{R}) \times A^{ \pm} \mapsto \lambda g \in \mathrm{GL}_{2}(\mathbb{R}) .
$$

In particular,

$$
H_{\mathbf{t}}:=\left[\begin{array}{cc}
\mathrm{GL}_{2}(\mathbb{R}) & 0 \\
0 & 1
\end{array}\right]
$$

is contained in the automorphism group of $\left(Y_{\mathbf{t}}, \mathcal{S}_{\mathbf{t}}\right)$.

4.2.3. The open subset $Y_{\mathbf{a}}$. The action of $\operatorname{Heis}(3)$ at $o_{\mathbf{a}}=\left(\left[e_{3}\right],\left[e_{3}, e_{2}\right]\right)=\phi_{a}^{-1}((0,0)+$ $\left.\mathbb{R} e_{2}\right) \in \Omega_{a}$ is simply transitive, and its orbit $Y_{\mathbf{a}}=\operatorname{Heis}(3) \cdot o_{\mathbf{a}}$ can be described as

$$
Y_{\mathbf{a}}=\Omega_{a} \backslash S_{\alpha, \beta}\left[e_{1}\right]=\phi_{a}^{-1}\left(\left\{m+L \mid m \in \mathbb{R}^{2}, L \in \mathbb{R} \mathbf{P}^{1} \backslash\left\{\mathbb{R} e_{1}\right\}\right\}\right) .
$$

We endow $Y_{\mathbf{a}}$ with the Heis(3)-invariant enhanced Lagrangian contact structure

$$
\mathcal{S}_{\mathbf{a}}=\left(\left.\theta_{O_{\mathbf{a}}}\right|_{\operatorname{Heis}(3)}\right)_{*} \mathcal{S}_{\mathrm{Heis}(3)}
$$

which is compatible with $\mathcal{L}_{\mathbf{X}}$, and whose central distribution is entirely determined by

$$
\mathcal{E}_{\mathbf{a}}^{c}\left(o_{\mathbf{a}}\right)=\mathbb{R} Z^{\dagger}\left(o_{\mathbf{a}}\right) .
$$

Let us recall that $\mathcal{A}$ is the subgroup of automorphisms of Heis(3) that moreover preserve $\mathcal{S}_{\text {Heis(3) }}$ (see Paragraph 4.1.2). The group of affine automorphisms $L_{g} \circ \varphi$ of Heis(3), where $g \in \operatorname{Heis}(3)$ and $\varphi \in \mathcal{A}$, will be seen as a semi-direct subgroup Heis $(3) \rtimes \mathcal{A}$. With this notation, the isomorphism from $\left(Y_{\mathbf{a}}, \mathcal{S}_{\mathbf{a}}\right)$ to Heis $(3)$ given by the orbital map at $o_{\mathbf{a}}$ is equivariant for the identification

$$
\left[\begin{array}{ccc}
\lambda & x & z \\
0 & \lambda^{-1} \mu^{-1} & y \\
0 & 0 & \mu
\end{array}\right] \in \mathbf{P}_{\text {min }} \mapsto\left(\left(\begin{array}{ccc}
1 & \lambda \mu x & \mu^{-1} z \\
0 & 1 & \mu^{-1} y \\
0 & 0 & 1
\end{array}\right), \varphi_{\lambda^{2} \mu, \lambda^{-1} \mu^{-2}}\right) \in \operatorname{Heis}(3) \rtimes \mathcal{A},
$$

and in particular, $H_{\mathbf{a}}:=\mathbf{P}_{\min }$ is contained in the automorphism group of $\left(Y_{\mathbf{a}}, \mathcal{S}_{\mathbf{a}}\right)$.

4.3. From the infinitesimal model to the local model. We take back the notations of Theorem B. We recall that $\pi_{M}: \tilde{M} \rightarrow M$ denotes the universal cover of $M$ and that $\Omega$ is a dense and open subset of $M$ where $\mathcal{S}$ is locally homogeneous (see Proposition 3.2). We will denote $\tilde{\mathcal{S}}=\pi_{M}^{*} \mathcal{S}=\left(\tilde{E}^{\alpha}, \tilde{E}^{\beta}, \tilde{E}^{c}\right), \tilde{\Omega}=\pi_{M}^{-1}(\Omega)$, and $\delta: \tilde{M} \rightarrow \mathbf{X}$ a developping map of the $(\mathbf{G}, \mathbf{X})$-structure of $M$ describing the Lagrangian contact structure $\mathcal{L}$ (see Corollary 3.4 and Paragraph 2.3.2). We finally choose for this whole section a connected component $O$ of $\tilde{\Omega}$, i.e. an open Kill ${ }^{l o c}$-orbit of $\tilde{\mathcal{S}}$.

Our goal in this section is to describe the local model of $\tilde{\mathcal{S}}$ in restriction to $O$. 
4.3.1. Infinitesimal model. At any point of $\mathbf{X}$, we will identify the Lie algebra of local Killing fields of $\mathcal{L}_{\mathbf{X}}$ with $\mathfrak{s l}_{3}$ through the fundamental vector fields of the action of $\mathbf{G}$ (see Lemma 2.16). Since the developping map $\delta$ is a local isomorphism from $\widetilde{\mathcal{L}}$ to $\mathcal{L}_{\mathbf{X}}$, it induces at each point $x \in \tilde{M}$ an isomorphism

$$
\delta^{*}: v \in \mathfrak{s l}_{3}=\mathfrak{k i r l}_{\mathcal{L}_{\mathbf{X}}}^{l o c}(\delta(x)) \mapsto \delta^{*} v \in \mathfrak{k i r l}_{\widetilde{\mathcal{L}}}^{l o c}(x),
$$

of Lie algebras, whose inverse will be denoted by $\delta_{*}: \mathfrak{k i l l}_{\widetilde{\mathcal{L}}}^{l o c}(x) \rightarrow \mathfrak{s l}_{3}$. For $X \in \mathfrak{k i f l l}_{\widetilde{\mathcal{L}}}^{l o c}(x)$ and $t \in \mathbb{R}$ for which $\varphi_{X}^{t}(x)$ exists, denoting $v=\delta_{*}[X]_{x} \in \mathfrak{s l}_{3}$, we have

$$
\delta\left(\varphi_{X}^{t}(x)\right)=\mathrm{e}^{t v} \cdot \delta(x) .
$$

Lemma 4.1. There exists a subalgebra $\mathfrak{h}$ of $\mathfrak{s l}_{3}$ such that

$$
\mathfrak{K i l l r}\left(O,\left.\tilde{\mathcal{S}}\right|_{O}\right)=\left.\left(\delta^{*} \mathfrak{h}\right)\right|_{O}=\left\{\left.\left(\delta^{*} v\right)\right|_{O} \mid v \in \mathfrak{h}\right\} .
$$

Moreover, any local Killing field of $\tilde{\mathcal{S}}$ on $O$ extends to the whole Kill ${ }^{l o c}$-orbit $O$.

Proof. It suffices to show that the subalgebra $\mathfrak{h}(x)=\delta_{*} \mathfrak{k i f l l}_{\tilde{\mathcal{S}}}^{\text {loc }}(x)$ is locally constant on $O$. This will in fact imply by connexity of $O$ that $\mathfrak{h}(x)$ is constant equal to some Lie subalgebra $\mathfrak{h}$ on $O$, and then $\left.\left(\delta^{*} \mathfrak{h}\right)\right|_{O} \subset \mathfrak{K i l l}\left(O,\left.\tilde{\mathcal{S}}\right|_{O}\right)$. But for $x \in O, \operatorname{dim} \mathfrak{h}=\operatorname{dim} \mathfrak{k i l l}{ }_{\tilde{\mathcal{S}}}^{l o c}(x) \geq$ $\mathfrak{K i t l}\left(O,\left.\tilde{\mathcal{S}}\right|_{O}\right)$ (see Lemma 2.14 ), and this inclusion is thus an equality.

For any $x \in O$ there exists an open connected neighbourhood $U$ of $x$ such that any local Killing field of $\tilde{\mathcal{S}}$ at $x$ extends to a Killing field defined on $U$ (see Remark 2.15), and for any $y \in U$ we have thus $\mathfrak{h}(x) \subset \mathfrak{h}(y)$. But $\mathfrak{h}(x)$ and $\mathfrak{h}(y)$ have the same dimension since $x$ and $y$ are in the same Kill ${ }^{\text {loc }}$-orbit of $\mathcal{\mathcal { S }}$, and this inclusion is thus an equality. This shows that $\mathfrak{h}(x)$ is locally constant and finishes the proof.

We denote from now on by $H$ the connected Lie subgroup of $\mathbf{G}$ of subalgebra $\mathfrak{h}$. It is not necessarily closed in $\mathbf{G}$, but the action of $H$ on $\mathbf{X}$ is smooth for the structure of immersed submanifold of $H$.

Lemma 4.2. All the points of $\delta(O)$ are in the same orbit $Y$ under the action of $H$. In particular, $Y$ is open.

Proof. We consider $x$ and $y$ in $O$, and we want to find $h \in H$ such that $\delta(y)=h \cdot \delta(x)$. By hypothesis, as $x$ and $y$ are in the same Kill ${ }^{\text {loc }}$-orbit of $\tilde{\mathcal{S}}$, there exists a finite number of points $x_{1}=x, \ldots, x_{n}=y$ such that for any $i \leq n-1$ there exists a local Killing field $X_{i}$ of $\tilde{\mathcal{S}}$ satisfying $x_{i+1}=\varphi_{X_{i}}^{1}\left(x_{i}\right)$. According to Lemma 4.1, there exists for each $i$ an element $v_{i} \in \mathfrak{h}$ such that $X_{i}=\delta^{*} v_{i}$, and we have $\delta\left(x_{i+1}\right)=\mathrm{e}^{v_{i}} \delta\left(x_{i}\right)$ according to the equation (4.11), implying $\delta(y)=\mathrm{e}^{v_{n-1}} \ldots \mathrm{e}^{v_{1}} x_{0} \in H \cdot \delta(x)$.

We choose from now on a point $x \in O$, we denote $x_{0}=\delta(x) \in Y$, and we consider the isotropy subalgebra

$$
\mathfrak{i}=\mathfrak{s t a b} \mathfrak{b}_{\mathfrak{h}}\left(x_{0}\right):=\left\{v \in \mathfrak{h} \mid v\left(x_{0}\right)=0\right\}
$$

of $\mathfrak{h}$ at $x_{0}$, characterized by $\delta^{*} \mathfrak{i}=\mathfrak{i s}_{\tilde{\mathcal{S}}}^{l o c}(x)$. Since the orbit $Y$ of $x_{0}$ under $H$ is open, $\operatorname{dim} \mathfrak{h}-\operatorname{dim} \mathfrak{i}=3$, and $\mathfrak{i}$ is non-trivial according to Proposition 3.2. We also denote $\mathcal{E}^{c}\left(x_{0}\right)=\mathrm{D}_{x} \delta\left(\tilde{E}^{c}(x)\right)$, and $\mathfrak{h} / \mathfrak{i}=D^{\alpha} \oplus D^{\beta} \oplus D^{c}$ the splitting sent to $\mathrm{T}_{x_{0}} Y=\left(\mathcal{E}^{\alpha} \oplus \mathcal{E}^{\beta} \oplus\right.$ $\left.\mathcal{E}^{c}\right)\left(x_{0}\right)$ by the isomorphism $\overline{\mathrm{D}_{e} \theta_{x_{0}}}$ induced by the orbital map at $x_{0}$. 
Lemma 4.3. (1) The adjoint representation $\overline{\mathrm{ad}}: \mathfrak{i} \rightarrow \mathrm{L}(\mathfrak{h} / \mathfrak{i})$ preserves the line $D^{c}$ in $\mathfrak{h} / \mathfrak{i}$, i.e. for any $v \in \mathfrak{i}$ we have $\overline{\operatorname{ad}}(v)\left(D^{c}\right) \subset D^{c}$.

(2) There exists in the neighbourhood of $x_{0}$ an unique $H$-invariant germ of a smooth one-dimensional distribution $\mathcal{E}^{c}$ that extends $\mathcal{E}^{c}\left(x_{0}\right)$ on a neighbourhood of $x_{0}$, and this distribution is everywhere transverse to $\mathcal{E}^{\alpha} \oplus \mathcal{E}^{\beta}$.

(3) The developping map $\delta$ is an isomorphism between the enhanced Lagrangian contact structures $\tilde{\mathcal{S}}$ and $\mathcal{S}_{Y}:=\left(\mathcal{E}^{\alpha}, \mathcal{E}^{\beta}, \mathcal{E}^{c}\right)$, from a neighbourhood of $x$ to a neighbourhood of $x_{0}$.

(4) $\mathfrak{h}=\mathfrak{k i r l l}_{\mathcal{S}_{Y}}^{\text {loc }}\left(x_{0}\right)$ and $\mathfrak{i}=\mathfrak{i s}_{\mathcal{S}_{Y}}^{\text {loc }}\left(x_{0}\right)$.

(5) If $I=\operatorname{Stab}_{H}\left(x_{0}\right)$ is a connected subgroup of $H$, then there exists an unique $H$-invariant smooth one-dimensional distribution $\mathcal{E}^{c}$ that extends $\mathcal{E}^{c}\left(x_{0}\right)$ on the whole open orbit $Y$, and $\mathcal{E}^{c}$ is transverse to $\mathcal{E}^{\alpha} \oplus \mathcal{E}^{\beta}$. Furthermore, $\left.\delta\right|_{O}$ is a local isomorphism from $\left(O,\left.\tilde{\mathcal{S}}\right|_{O}\right)$ to $\left(Y, \mathcal{S}_{Y}\right)$.

Proof. 1. For $v \in \mathfrak{i}$, denoting $X=\delta^{*} v \in \mathfrak{i s}_{\tilde{\mathcal{S}}}^{\text {loc }}(x)$, equation (4.11) implies $\mathcal{E}^{c}\left(x_{0}\right)=$ $\mathrm{D}_{x_{0}} \mathrm{e}^{t v}\left(\mathcal{E}^{c}\left(x_{0}\right)\right)$ for any $t \in \mathbb{R}$, and thus $D^{c}=\overline{\operatorname{Ad}}\left(\mathrm{e}^{t v}\right) \cdot D^{c}=\exp (\overline{\operatorname{ad}}(v)) \cdot D^{c}$. Derivating this last equality at $t=0$, we obtain $\overline{\operatorname{ad}}(v) \cdot D^{c} \subset D^{c}$.

2. The group $I=\operatorname{Stab}_{H}\left(x_{0}\right)$ and its identity component $I^{0}$ are closed in $H$ for its topology of immersed submanifold, and the orbital map at $x_{0}$ induces a local diffeomorphism $\bar{\theta}_{x_{0}}: H / I^{0} \rightarrow Y$, equivariant for the action of $H$. We saw previously that $\overline{\operatorname{Ad}}(\exp (\mathfrak{i}))$ preserves $D^{c}$, implying that the subgroup $\left\{i \in I^{0} \mid \overline{\operatorname{Ad}}(i) \cdot D^{c}=D^{c}\right\}$ is equal to $I^{0}$ by connexity, i.e. that $I^{0}$ preserves $D^{c}$. Therefore, $H / I^{0}$ supports an unique $H$-invariant smooth one-dimensional distribution extending $D^{c}$, that can be pushed by $\bar{\theta}_{x_{0}}: H / I^{0} \rightarrow Y$, to a $H$-invariant distribution extending $\mathcal{E}^{c}\left(x_{0}\right)$ on a neighbourhood of $x_{0}$. Conversely, the pullback of any $H$-invariant distribution extending $\mathcal{E}^{c}\left(x_{0}\right)$ on a neighbourhood of $x_{0}$ is $H$-invariant on $H / I^{0}$, which proves the unicity of the germ of $\mathcal{E}^{c}$. As it is preserved by $H$, it must remain transverse to $\mathcal{E}^{\alpha} \oplus \mathcal{E}^{\beta}$.

3. For $y$ sufficiently close to $x$, there exists $X \in \mathfrak{K i r l}\left(O,\left.\tilde{\mathcal{S}}\right|_{O}\right)$ such that $y=\varphi_{X}^{1}(x)$. Denoting $y_{0}=\delta(y)$ and $v \in \mathfrak{h}$ such that $\delta^{*} v=X$, we have $\mathrm{D}_{y_{0}} \mathrm{e}^{-v} \circ \mathrm{D}_{y} \delta\left(\tilde{E}^{c}(y)\right)=$ $\mathrm{D}_{x} \delta \circ \mathrm{D}_{y} \varphi_{X}^{-1}\left(\tilde{E}^{c}(y)\right)=\mathcal{E}^{c}\left(x_{0}\right)$, implying $\mathrm{D}_{y} \delta\left(\tilde{E}^{c}(y)\right)=\mathcal{E}^{c}\left(y_{0}\right)$ by $H$-invariance of $\mathcal{E}^{c}$.

4. This is a direct consequence of $\delta^{*} \mathfrak{h}=\mathfrak{k i f l}_{\tilde{\mathcal{S}}}^{\text {loc }}(x), \delta^{*} \mathfrak{i}=\mathfrak{i s}_{\tilde{\mathcal{S}}}^{\text {loc }}(x)$, and of the fact that $\delta$ is a local isomorphism from $\tilde{\mathcal{S}}$ to $\mathcal{S}_{Y}$ at $x$.

5. Concerning the first assertion, the orbital map at $x_{0}$ induces a $H$-equivariant diffeomorphism from $H / I$ to $Y$, and we saw in the proof of the second assertion that $H / I^{0}=H / I$ supports an unique $H$-invariant distribution extending $D^{c}$ on $H / I^{0}$, which stays transverse to the contact plane.

The set $\mathcal{E}$ of points $y \in O$ such that $\delta$ is a local isomorphism in the neighbourhood of $y$ is open and non-empty, and we only have to prove that $\mathcal{E}$ is closed to conclude by connexity of $O$. Let $z \in O$ be an adherent point of $\mathcal{E}$, and let us denote $z_{0}=\delta(z)$. There exists a point $y \in \mathcal{E}$ sufficiently close to $z$ such that, for some Killing field $X$ of $\tilde{\mathcal{S}}, z=\varphi_{X}^{1}(y)$. Denoting $v \in \mathfrak{h}$ such that $X=\delta^{*} v$, we have $\mathrm{D}_{z_{0}} \mathrm{e}^{-v} \circ \mathrm{D}_{z} \delta\left(\tilde{E}^{c}(z)\right)=$ $\mathrm{D}_{y} \delta \circ \mathrm{D}_{z} \varphi_{X}^{-1}\left(\tilde{E}^{c}(z)\right)=\mathcal{E}^{c}\left(y_{0}\right)$, implying $\mathrm{D}_{z} \delta\left(\tilde{E}^{c}(z)\right)=\mathcal{E}^{c}\left(z_{0}\right)$ by $H$-invariance of $\mathcal{E}^{c}$. By local homogeneity of $\left.\mathcal{S}\right|_{O}$, we can reach all the points of some neighbourhood $U$ of $z$ in $O$ by a Killing field, and the same computation as before shows that $\left.\delta\right|_{U}$ is a local isomorphism, i.e. that $z \in \mathcal{E}$. 
4.3.2. Local model of an open Kill ${ }^{l o c}$-orbit. We will call

$$
\kappa:(m, D) \in \mathbf{X} \mapsto\left(D^{\perp}, m^{\perp}\right) \in \mathbf{X}
$$

the flip diffeomorphism of the homogeneous model space. This involution switches the distributions $\mathcal{E}^{\alpha}$ and $\mathcal{E}^{\beta}$ of the standard Lagrangian contact structure, and is moreover equivariant for the Lie group morphism $\Theta: g \mapsto{ }^{t} g^{-1}$ of $\mathbf{G}$.

Consequently, interverting the distributions $E^{\alpha}$ and $E^{\beta}$ of the Lagrangian contact structure of $M$ is equivalent to composing the developping map $\delta$ with $\kappa$. At the level of the subalgebra $\mathfrak{h}$ introduced in the previous paragraph, it is equivalent to apply the Lie algebra morphism $\mathrm{D}_{e} \Theta=\theta: A \mapsto-{ }^{t} A$.

Denoting

$$
\text { (4.14) }\left\{\begin{array}{l}
\mathfrak{h}_{\mathbf{t}}=\left\{\left(\begin{array}{cc}
A & 0 \\
0 & -\operatorname{tr}(A)
\end{array}\right) \mid A \in \mathfrak{g l}_{2}\right\}, \\
\mathfrak{h}_{\mathbf{a}}=\mathfrak{p}_{\text {min }},
\end{array}\right.
$$

we will prove in the next section that:

Proposition 4.4. Up to conjugacy in $\mathbf{G}$ or image by $\theta=-{ }^{t}$, $\mathfrak{h}$ is equal to $\mathfrak{h}_{\mathbf{t}}$ or $\mathfrak{h}_{\mathbf{a}}$.

To deduce a local information about $\left.\tilde{\mathcal{S}}\right|_{O}$ from this infinitesimal classification, it only remains to look at the action of the connected Lie subgroups $H_{\mathbf{t}}^{0}:=\mathrm{GL}_{2}^{+}(\mathbb{R})$ and $H_{\mathbf{a}}^{0}=$ $\mathbf{P}_{\text {min }}^{+}$of $\mathbf{G}$, of respective Lie algebras $\mathfrak{h}_{\mathbf{t}}$ and $\mathfrak{h}_{\mathbf{a}}$.

Proposition 4.5. (1) $Y_{\mathbf{t}}$ (respectively $Y_{\mathbf{a}}$ ) is the only open orbit of $H_{\mathbf{t}}^{0}$ (resp. of $\left.H_{\mathbf{a}}^{0}\right)$ on $\mathbf{X}$.

(2) $\mathcal{S}_{\mathbf{t}}$ (respectively $\mathcal{S}_{\mathbf{a}}$ ) is the only $H_{\mathbf{t}}^{0}$-invariant (resp. $H_{\mathbf{a}}^{0}$-invariant) enhanced Lagrangian contact structure of $Y_{\mathbf{t}}$ (resp. $Y_{\mathbf{a}}$ ) that is compatible with $\mathcal{L}_{\mathbf{X}}$.

Proof. 1. Both of these groups are contained in $\operatorname{Stab}_{\mathbf{G}}\left[e_{1}, e_{2}\right]=\left\{\left[\begin{array}{cc}A & X \\ 0 & 1\end{array}\right] \mid A \in \mathrm{GL}_{2}(\mathbb{R}), X \in\right.$ $\left.\mathbb{R}^{2}\right\}$, that preserves the surface $S_{\beta, \alpha}\left[e_{1}, e_{2}\right]$, and whose only open orbit is thus $\Omega_{a}=$ $\mathbf{X} \backslash S_{\beta, \alpha}\left[e_{1}, e_{2}\right]$. Any open orbit of one these groups is therefore contained in $\Omega_{a}$. Since $H_{\mathbf{t}}^{0}$ preserves the surface $S_{\alpha, \beta}\left[e_{3}\right]$, any open orbit of $H_{\mathbf{t}}^{0}$ is contained in $Y_{\mathbf{t}}=$ $\mathbf{X} \backslash\left(S_{\beta, \alpha}\left[e_{1}, e_{2}\right] \cup S_{\alpha, \beta}\left[e_{3}\right]\right)=H_{\mathbf{t}}^{0} \cdot o_{\mathbf{t}}$. In the same way, since $H_{\mathbf{a}}^{0}$ preserves $S_{\alpha, \beta}\left[e_{1}\right]$, any open orbit of $H_{\mathbf{a}}^{0}$ is contained in $Y_{\mathbf{a}}=\mathbf{X} \backslash\left(S_{\beta, \alpha}\left[e_{1}, e_{2}\right] \cup S_{\alpha, \beta}\left[e_{1}\right]\right)=H_{\mathbf{a}}^{0} \cdot o_{\mathbf{a}}$.

2. We start with $Y_{\mathbf{t}}$, and we denote

$$
\mathfrak{i}_{\mathbf{t}}=\operatorname{Lie}\left(\operatorname{Stab}_{H_{\mathbf{t}}^{0}}\left(o_{\mathbf{t}}\right)\right)=\left\{\left(\begin{array}{ccc}
a & 0 & 0 \\
0 & -2 a & 0 \\
0 & 0 & a
\end{array}\right) \mid a \in \mathbb{R}\right\}
$$

and

$$
E=\left(\begin{array}{lll}
0 & 1 & 0 \\
0 & 0 & 0 \\
0 & 0 & 0
\end{array}\right), F=\left(\begin{array}{lll}
0 & 0 & 0 \\
1 & 0 & 0 \\
0 & 0 & 0
\end{array}\right), H=\left(\begin{array}{ccc}
1 & 0 & 0 \\
0 & -1 & 0 \\
0 & 0 & 0
\end{array}\right) .
$$

The standard Lagrangian contact structure of $\mathbf{X}$ satisfies $\mathbb{R} E^{\dagger}\left(o_{\mathbf{t}}\right)=\mathcal{E}^{\alpha}\left(o_{\mathbf{t}}\right)$ and $\mathbb{R} F^{\dagger}\left(o_{\mathbf{t}}\right)=$ $\mathcal{E}^{\beta}\left(o_{\mathbf{t}}\right)$, and for $a \in \mathbb{R}$, the adjoint action of the diagonal element $[a,-2 a, a]$ of $\mathfrak{i}_{\mathbf{t}}$ has the following diagonal matrix in the basis $(\bar{E}, \bar{F}, \bar{H})$ of $\mathfrak{h} / \mathfrak{i}$ :

$$
\operatorname{Mat}_{(\bar{E}, \bar{F}, \bar{H})}(\overline{\operatorname{ad}}([a,-2 a, a]))=[3 a,-3 a, 0] .
$$

Any line $D^{c}$ of $\mathfrak{h}_{\mathbf{t}} / \mathfrak{i}_{\mathbf{t}}$ that is transverse to $\operatorname{Vect}(\bar{E}, \bar{F})$ has projective coordinates $[x, y, 1]$ in the basis $(\bar{E}, \bar{F}, \bar{H})$ for some $(x, y) \in \mathbb{R}^{2}$, and $\overline{\operatorname{ad}}([a,-2 a, a])\left(D^{c}\right)$ is therefore generated 
by the vector of coordinates $(3 a x,-3 a y, 0)$. The only transverse line stabilized by $\overline{\operatorname{ad}}\left(\mathfrak{i}_{\mathbf{t}}\right)$ is therefore $\mathbb{R} \bar{H}$, and $\mathcal{E}_{\mathbf{t}}^{c}$ is the only $H_{\mathbf{t}}^{0}$-invariant distribution of $Y_{\mathbf{t}}$ transverse to $\mathcal{L}_{\mathbf{X}}$.

Let us denote

$$
\mathfrak{i}_{\mathbf{a}}=\operatorname{Lie}\left(\operatorname{Stab}_{H_{\mathbf{a}}^{0}}\left(o_{\mathbf{a}}\right)\right)=\left\{\left(\begin{array}{ccc}
a & 0 & 0 \\
0 & -a-b & 0 \\
0 & 0 & b
\end{array}\right) \mid(a, b) \in \mathbb{R}^{2}\right\} .
$$

and

$$
X=\left(\begin{array}{lll}
0 & 1 & 0 \\
0 & 0 & 0 \\
0 & 0 & 0
\end{array}\right), Y=\left(\begin{array}{lll}
0 & 0 & 0 \\
0 & 0 & 1 \\
0 & 0 & 0
\end{array}\right), Z=\left(\begin{array}{lll}
0 & 0 & 1 \\
0 & 0 & 0 \\
0 & 0 & 0
\end{array}\right) .
$$

We have $\mathbb{R} X^{\dagger}\left(o_{\mathbf{a}}\right)=\mathcal{E}^{\alpha}\left(o_{\mathbf{a}}\right), \mathbb{R} Y^{\dagger}\left(o_{\mathbf{a}}\right)=\mathcal{E}^{\beta}\left(o_{\mathbf{a}}\right)$, and for $(a, b) \in \mathbb{R}^{2}$, the adjoint action of the diagonal element $[a,-a-b, b]$ of $\mathfrak{i}_{\mathbf{a}}$ has the following diagonal matrix in the basis $(\bar{X}, \bar{Y}, \bar{Z})$ of $\mathfrak{p}_{\min } / \mathfrak{i}_{\mathbf{a}}$

$$
\operatorname{Mat}_{(\bar{X}, \bar{Y}, \bar{Z})}(\overline{\operatorname{ad}}([a,-a-b, b]))=[2 a+b,-a-2 b, a-b] .
$$

Any line $D^{c}$ of $\mathfrak{p}_{\min } / \mathfrak{i}_{\mathbf{a}}$ that is transverse to $\operatorname{Vect}(\bar{X}, \bar{Y})$ has projective coordinates of the form $[x, y, 1]$ in the basis $(\bar{X}, \bar{Y}, \bar{Z})$ for some $(x, y) \in \mathbb{R}^{2}$, and $\overline{\operatorname{ad}}([a,-a-b, b])\left(D^{c}\right)$ is therefore generated by the vector of coordinates $((2 a+b) x,(-a-2 b) y, a-b)$. The only transverse line stabilized by $\overline{\mathrm{ad}}\left(\mathfrak{i}_{\mathbf{a}}\right)$ is therefore $\mathbb{R} \bar{Z}$, and $\mathcal{E}_{\mathbf{a}}^{c}$ is the only $H_{\mathbf{a}}^{0}$-invariant distribution of $Y_{\mathbf{a}}$ transverse to $\mathcal{L}_{\mathbf{X}}$.

We can finally describe the local geometry of $O$, which is a connected component of $\tilde{\Omega}=\pi_{M}^{-1}(\Omega)$.

Corollary 4.6. Up to inversion of the distributions $E^{\alpha}$ and $E^{\beta}$, the restriction $\left.\delta\right|_{O}$ of the developping map to $O$ is a local isomorphism from $\left(O,\left.\tilde{\mathcal{S}}\right|_{O}\right)$ to $\left(Y_{\mathbf{t}}, \mathcal{S}_{\mathbf{t}}\right)$, or to $\left(Y_{\mathbf{a}}, \mathcal{S}_{\mathbf{a}}\right)$.

Proof. The inversion of the distributions $E^{\alpha}$ and $E^{\beta}$ is equivalent to apply $\theta$ to $\mathfrak{h}$, and the conjugation of $\mathfrak{h}$ by $g \in \mathbf{G}$ is equivalent to replace the developping map $\delta$ by $g \circ \delta$ (that describes the same $(\mathbf{G}, \mathbf{X})$-structure on $M$ ). According to Proposition 4.4, we can thus assume that $\mathfrak{h}$ is equal to $\mathfrak{h}_{\mathbf{t}}$ or $\mathfrak{h}_{\mathbf{a}}$, and the open orbit $Y$ is therefore equal to $Y_{\mathbf{t}}$ (respectively $Y_{\mathbf{a}}$ ) according to Proposition 4.5. Since the isotropy subgroups $\operatorname{Stab}_{H_{\mathbf{t}}^{0}}\left(o_{\mathbf{t}}\right)$ and $\operatorname{Stab}_{H_{\mathbf{a}}^{0}}\left(o_{\mathbf{a}}\right)$ are connected, there exists a $H_{\mathbf{t}}^{0}$-invariant (resp. $H_{\mathbf{a}}^{0}$-invariant) enhanced Lagrangian contact structure $\mathcal{S}_{Y}$ on $Y$ that is compatible with $\mathcal{L}_{\mathbf{X}}$ and such that $\left.\delta\right|_{O}$ is a local isomorphism from $\left(O,\left.\tilde{\mathcal{S}}\right|_{O}\right)$ to $\left(Y, \mathcal{S}_{Y}\right)$ (see Lemma 4.3). According to Proposition $4.5, \mathcal{S}_{Y}$ is equal to $\mathcal{S}_{\mathbf{t}}\left(\right.$ resp. $\left.\mathcal{S}_{\mathbf{a}}\right)$.

\section{Classification of the infinitesimal model}

The goal of this section is to prove Proposition 4.4. Let us recall that the Lie subalgebras $\mathfrak{i} \subset \mathfrak{h}$ of $\mathfrak{s l}_{3}$ are characterized by $\left.\left(\delta^{*} \mathfrak{h}\right)\right|_{O}=\mathfrak{K i l l}\left(O,\left.\tilde{\mathcal{S}}\right|_{O}\right)$ and $\left[\delta^{*} \mathfrak{i}\right]_{x}=\mathfrak{i s}_{\tilde{\mathcal{S}}}^{\text {loc }}(x)$ (see Lemma 4.1 and (4.12)).

5.1. Algebraic reduction. We first prove some purely algebraic restrictions on $\mathfrak{h}$.

Lemma 5.1. The dimension of $\mathfrak{h}$ is either 4 or 5 .

Proof. Possibly translating the developping map by an element of $\mathbf{G}$, we can assume that $x_{0}=o=\left(\left[e_{1}\right],\left[e_{1}, e_{2}\right]\right) \in \mathbf{X}$, and since the adjoint action of $\mathbf{P}_{\min }$ on the lines of $\mathfrak{s l}_{3} / \mathfrak{p}_{\text {min }}$ transverse to $\operatorname{Vect}\left(\bar{e}_{\alpha}, \bar{e}_{\beta}\right)$ is transitive (see Paragraph 2.2), we can moreover 
assume that $D^{c}=\overline{\mathrm{D}_{e} \theta_{o}}\left(\mathbb{R} \bar{e}_{0}\right)$ with $e_{0}=\left(\begin{array}{lll}0 & 0 & 0 \\ 0 & 0 & 0 \\ 1 & 0 & 0\end{array}\right)$. As a consequence, $\mathfrak{i}=\mathfrak{h} \cap \mathfrak{p}_{\text {min }}$ is contained in

$$
\mathfrak{o}=\left\{v \in \mathfrak{p}_{\text {min }} \mid \overline{\operatorname{ad}}(v)\left(\mathbb{R} \bar{e}_{0}\right) \subset \mathbb{R} \bar{e}_{0}\right\}=\left\{\left(\begin{array}{ccc}
a & 0 & z \\
0 & -a-b & 0 \\
0 & 0 & b
\end{array}\right) \mid(a, b, z) \in \mathbb{R}^{3}\right\} .
$$

Denoting $e^{0}=\left(\begin{array}{lll}0 & 0 & 1 \\ 0 & 0 & 0 \\ 0 & 0 & 0\end{array}\right) \in \mathfrak{o}$, we now prove that $\mathfrak{i} \cap \mathbb{R} e^{0}=\{0\}$, implying $\operatorname{dim} \mathfrak{i} \leq 2$ and finishing the proof of the Lemma, since $\mathfrak{i}$ is non-zero and $\operatorname{dim} \mathfrak{h}-\operatorname{dim} \mathfrak{i}=3$.

Let us assume by contradiction that $e^{0} \in \mathfrak{i}$. As $\mathfrak{h}+\mathfrak{p}_{\text {min }}=\mathfrak{s l}_{3}$ (because the orbit of $o$ under $H$ is open), there exists $v \in \mathfrak{h}$ and $w \in \mathfrak{p}_{\text {min }}$ such that $e_{\beta}=\left(\begin{array}{lll}0 & 0 & 0 \\ 1 & 0 & 0 \\ 0 & 0 & 0\end{array}\right)=v+w$. But $\left[v, e^{0}\right] \in \mathfrak{h}$, and $\left[w, e^{0}\right] \in \mathbb{R} e^{0} \subset \mathfrak{i}$ since $w \in \mathfrak{p}_{\text {min }}$, finally implying that $\left(\begin{array}{lll}0 & 0 & 0 \\ 0 & 0 & 1 \\ 0 & 0 & 0\end{array}\right)=$ $\left[e_{\beta}, e^{0}\right]=\left[v, e^{0}\right]+\left[w, e^{0}\right] \in \mathfrak{h} \cap \mathfrak{p}_{\text {min }}=\mathfrak{i} \subset \mathfrak{o}$, which contradicts the description of $\mathfrak{o}$ in $(5.1)$.

Let

$$
\mathfrak{h}=\mathfrak{s} \ltimes_{\phi} \mathfrak{r}
$$

be the Levi decomposition of $\mathfrak{h}$, where $\mathfrak{s}$ is a semi-simple subalgebra of $\mathfrak{h}$ (or is trivial if $\mathfrak{h}$ is solvable), $\mathfrak{r}$ is the solvable radical of $\mathfrak{h}$ (it is an ideal of $\mathfrak{h}$ ), and $\phi$ is the restriction of the adjoint representation ad: $\mathfrak{h} \rightarrow$ Der $\mathfrak{h}(\phi: \mathfrak{s} \rightarrow$ Der $\mathfrak{r}$ describes the bracket in $\mathfrak{h}$ by $[v, w]=\phi(v)(w)$ for $v \in \mathfrak{s}$ and $w \in \mathfrak{r})$.

A proper semi-simple subalgebra of $\mathfrak{s l}_{3}$ of dimension less than 5 is three-dimensional, and is thus isomorphic to $\mathfrak{s l}_{2}$ or to $\mathfrak{s o}(3)$. Moreover, up to conjugacy in $\mathrm{SL}_{3}(\mathbb{R})$, the only embedding of $\mathfrak{s o}(3)$ in $\mathfrak{s l}_{3}$ is the inclusion, and the only embeddings of $\mathfrak{s l}_{2}$ in $\mathfrak{s l}_{3}$ are

$$
\mathfrak{s}_{0}:=\left\{\left(\begin{array}{cc}
A & 0 \\
0 & 0
\end{array}\right) \mid A \in \mathfrak{s l}_{2}\right\} \text { and } \mathfrak{s o}(1,2)
$$

If $\mathfrak{h}$ is not solvable, $\mathfrak{s}$ is thus equal to $\mathfrak{s}_{0}, \mathfrak{s o}(1,2)$ or $\mathfrak{s o}(3)$ up to conjugacy in $\mathrm{SL}_{3}(\mathbb{R})$. The centralizers of these subalgebras in $\mathfrak{s l}_{3}$ are

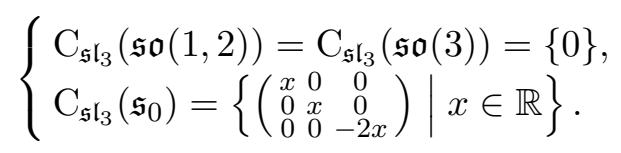

Lemma 5.2. Up to conjugacy in $\mathrm{SL}_{3}(\mathbb{R})$ or image by $\theta=-{ }^{t}$, we have the following results.

(1) If $\mathfrak{h}$ is not solvable, then
(a) $\mathfrak{s}$ is equal to $\mathfrak{s}_{0}$,
(b) and $\mathfrak{h}$ is equal to $\mathfrak{h}_{\mathbf{t}}$ or to

$$
\mathfrak{h}_{1}=\mathbb{R}^{2} \rtimes \mathfrak{s l}_{2}=\left\{\left(\begin{array}{cc}
A & X \\
0 & 0
\end{array}\right) \mid A \in \mathfrak{s l}_{2}, X \in \mathbb{R}^{2}\right\} .
$$

(2) If $\mathfrak{h}$ is solvable, then either $\mathfrak{h}$ is contained in $\mathfrak{h}_{\mathbf{a}}=\mathfrak{p}_{\text {min }}$, or equal to

$$
\begin{aligned}
& \qquad \mathfrak{h}_{2}=\mathbb{R}^{2} \rtimes \mathfrak{s i m}\left(\mathbb{R}^{2}\right)=\left\{\left(\begin{array}{cc}
A & X \\
0 & -\operatorname{tr} A
\end{array}\right) \mid A \in \mathfrak{s i m}\left(\mathbb{R}^{2}\right), X \in \mathbb{R}^{2}\right\}, \\
& \text { where } \mathfrak{s i m}\left(\mathbb{R}^{2}\right)=\left\{\left(\begin{array}{cc}
a & b \\
-b & a
\end{array}\right) \mid(a, b) \in \mathbb{R}^{2}\right\} .
\end{aligned}
$$


Proof. 1.a) Let us assume by contradiction that $\mathfrak{s}$ is conjugated to $\mathfrak{s o}(1,2)$ or $\mathfrak{s o}(3)$, implying that $\mathrm{C}_{\mathfrak{s l}_{3}} \mathfrak{s}=\{0\}$ according to (5.4). Since $\mathfrak{s}$ is simple, if the Lie algebra morphism $\phi$ is not injective then it is trivial, implying $\mathfrak{r} \subset \mathrm{C}_{\mathfrak{s l}_{3}} \mathfrak{s}=\{0\}$ and thus $\operatorname{dim} \mathfrak{h}=$ $\operatorname{dim} \mathfrak{s}=3$ which contradicts Lemma 5.1. Our hypothesis on $\mathfrak{s}$ implies therefore that $\phi$ is injective, and in particular that $\operatorname{dim} \operatorname{Der} \mathfrak{r} \geq \operatorname{dim} \mathfrak{s}=3$.

Since $\operatorname{dim} \mathfrak{s}=3$, the solvable radical $\mathfrak{r}$ is of dimension 1 or 2 according to Lemma 5.1 , and is thus isomorphic to $\mathbb{R}, \mathfrak{a f f}(\mathbb{R})$, or $\mathbb{R}^{2}$. But if $\mathfrak{r}$ is isomorphic to $\mathbb{R}$ or $\mathfrak{a f f}(\mathbb{R})$, then Der $\mathfrak{r}$ is of dimension 1 or 2 which contradicts the injectivity of $\phi$, and $\mathfrak{r}$ is thus isomorphic to $\mathbb{R}^{2}$. Since $\mathfrak{s o}(3)$ has no non-zero two-dimensional representation, this implies that $\mathfrak{s}$ is conjugated to $\mathfrak{s o}(1,2)$. The connected Lie subgroup $H$ of $\mathrm{SL}_{3}(\mathbb{R})$ of Lie algebra $\mathfrak{h}$ contains then $\mathrm{SO}^{0}(1,2)$, and its adjoint action induces thus by restriction a two-dimensional representation $\phi$ of $\mathrm{SO}^{0}(1,2)$ on $\mathfrak{r}$ (because $\mathfrak{r}$ is an ideal of $\mathfrak{h}$ ). Since $\mathrm{SO}^{0}(1,2)$ is isomorphic to $\mathrm{PSL}_{2}(\mathbb{R}), \phi$ is trivial, implying that $\phi$ is trivial as well, which contradicts the injectivity of $\phi$. Finally, $\mathfrak{s}$ is conjugated to $\mathfrak{s}_{0}$.

1.b) Let us assume by contradiction that $\mathfrak{r}$ is isomorphic to $\mathfrak{a f f}(\mathbb{R})$. Then Der $\mathfrak{r}$ is twodimensional and $\phi$ is thus non-injective, i.e. trivial by simplicity of $\mathfrak{s}_{0}$. But $\mathfrak{r}$ is then contained in the centralizer of $\mathfrak{s}_{0}$ which is one-dimensional according to (5.4), contradicting the original hypothesis. Therefore, $\mathfrak{r}$ is isomorphic to $\mathbb{R}^{2}$ or $\mathbb{R}$.

We first assume that $\mathfrak{r}$ is isomorphic to $\mathbb{R}^{2}$, implying that $\phi$ is injective (otherwise $\mathfrak{r} \subset \mathrm{C}_{\mathfrak{s l}_{3}} \mathfrak{s}_{0}$ which is one-dimensional). We use the linear mapping ev $\left.e_{e_{3}}\right|_{\mathfrak{r}}: M \in \mathfrak{r} \mapsto$ $M\left(e_{3}\right) \in \mathbb{R}^{3}$ and discuss according to the dimension of its image $\mathfrak{r}\left(e_{3}\right)$. Let us emphasize that $\mathfrak{r}$ is normalized by the connected Lie subgroup $S_{0}$ of $\mathrm{SL}_{3}(\mathbb{R})$ of Lie algebra $\mathfrak{s}_{0}$, and that $\mathfrak{r}\left(e_{3}\right)$ is thus preserved by $S_{0}$. If $\mathfrak{r}\left(e_{3}\right)$ is a plane then $\mathfrak{r}\left(e_{3}\right)=\operatorname{Vect}\left(e_{1}, e_{2}\right)$ since it is preserved by $S_{0}$, and $\mathrm{ev}_{e_{3}} \mid \mathfrak{r}$ is moreover injective. There exists $v \in \mathfrak{r}$ such that $\operatorname{ev}_{e_{3}}(v)=$ $e_{1}$, and with $A=\left(\begin{array}{cc}1 & 0 \\ 0 & -1\end{array}\right) \in \mathfrak{s l}_{2}$ and $u=\left(\begin{array}{cc}A & 0 \\ 0 & 0\end{array}\right) \in \mathfrak{s}_{0}$ we have $\operatorname{ev}_{e_{3}}([u, v])=e_{1}=\operatorname{ev}_{e_{3}}(v)$. This implies $[u, v]=v$ by injectivity of $\mathrm{ev}_{e_{3}} \mid \mathfrak{r}$, and finally $v=\left(\begin{array}{ccc}0 & 0 & 1 \\ 0 & 0 & 0 \\ 0 & x & 0\end{array}\right)$ for some $x \in \mathbb{R}$. The same reasoning with $w \in \mathfrak{r}$ such that $\mathrm{ev}_{e_{3}}(w)=e_{2}$ and $A=\left(\begin{array}{cc}-1 & 0 \\ 0 & 1\end{array}\right) \in \mathfrak{s l}_{2}$, implies that $w=\left(\begin{array}{lll}0 & 0 & 0 \\ 0 & 0 & 1 \\ y & 0 & 0\end{array}\right)$ for some $y \in \mathbb{R}$. Since $\mathfrak{r}$ is abelian we have $[v, w]=0$, which implies $x=y=0$ and proves that $\mathfrak{r}=\left(\begin{array}{cc}0 & \mathbb{R}^{2} \\ 0 & 0\end{array}\right)$, i.e. that $\mathfrak{h}=\mathbb{R}^{2} \rtimes \mathfrak{s l}_{2}$. If $\mathfrak{r}\left(e_{3}\right)=\{0\}$, then $\left.p\right|_{\mathfrak{r}}$ is injective, implying $p(\mathfrak{r})=\mathbb{R}^{2}$. Therefore $\operatorname{dim}(\theta(\mathfrak{r}))\left(e_{3}\right)=2$ which brings us back to the first case, and $\theta(\mathfrak{h})=\mathbb{R}^{2} \rtimes \mathfrak{s l}_{2}$. Finally, $\operatorname{dim} \mathfrak{r}\left(e_{3}\right)=1$ is impossible. Otherwise, $\mathfrak{r}^{\prime}=\operatorname{ker~ev}_{e_{3}} \cap \mathfrak{r}$ is one-dimensional, and since $p:\left(\begin{array}{cc}B & 0 \\ X & 0\end{array}\right) \in \mathfrak{r}^{\prime} \mapsto X \in \mathbb{R}^{2}$ is injective (because $\left.\mathfrak{r} \cap \mathfrak{s}_{0}=\{0\}\right), p\left(\mathfrak{r}^{\prime}\right)$ is a line of $\mathbb{R}^{2}$. But for $w \in \mathfrak{r}^{\prime}$ and $v=\left(\begin{array}{cc}A & 0 \\ 0 & 0\end{array}\right) \in \mathfrak{s}_{0}$ we have $p([v, w])=-p(w) A$, i.e. $p\left(\mathfrak{r}^{\prime}\right)$ is preserved by $\mathfrak{s l}_{2}$ and cannot be a line.

We now assume that $\mathfrak{r}$ is isomorphic to $\mathbb{R}$. Then $\phi$ is non-injective and thus trivial, implying $\mathfrak{r} \subset \mathrm{C}_{\mathfrak{s l}_{3} \mathfrak{s}_{0}}$. This inclusion is an equality by equality of dimensions, proving $\mathfrak{h}=\mathfrak{h}_{\mathbf{t}}$.

2. As $\mathfrak{h}$ is solvable, it preserves a complex line in $\mathbb{C}^{3}$ according to Levi's theorem. More precisely, either $\mathfrak{h}$ preserves a real line, or it preserves a plane on which it acts by similarities. The second case implies $\mathfrak{h} \subset \mathbb{R}^{2} \rtimes \mathfrak{s i m}\left(\mathbb{R}^{2}\right)=\mathfrak{h}_{2}$ up to conjugacy in $\mathrm{SL}_{3}(\mathbb{R})$. In the first case we can assume that $\mathfrak{h}$ preserves $\mathbb{R} e_{1}$, and if the representation $\left(\begin{array}{cc}* & * \\ 0 & A\end{array}\right) \in \mathfrak{h} \mapsto A \in \mathfrak{g l}_{2}$ also preserves a real line, then $\mathfrak{h} \subset \mathfrak{p}_{\text {min }}=\mathfrak{h}_{\mathbf{a}}$ up to conjugacy. If not, then $\theta(\mathfrak{h}) \subset\left\{\left(\begin{array}{cc}-\operatorname{tr} A & 0 \\ X & A\end{array}\right) \mid A \in \mathfrak{s i m}\left(\mathbb{R}^{2}\right), X \in \mathbb{R}^{2}\right\}$, according to the same remark 
than before. This last subalgebra being conjugated to $\mathbb{R}^{2} \rtimes \mathfrak{s i m}\left(\mathbb{R}^{2}\right)=\mathfrak{h}_{2}$, this concludes the proof of the lemma.

5.2. Two further properties of the infinitesimal model. We now prove two further properties of the infinitesimal model $(\mathfrak{h}, \mathfrak{i})$, in order to eliminate the "exotic" cases $\mathfrak{h}_{1}$ and $\mathfrak{h}_{2}$ that appeared in the algebraic clasification of Lemma 5.2.

Lemma 5.3. Let $\mathfrak{l}$ be a subalgebra of $\mathfrak{s l}_{3}$ containing $\mathfrak{h}, \mathfrak{j}=\mathfrak{s t a b}_{\mathfrak{l}}\left(x_{0}\right)$ be the isotropy at $x_{0}$, and $D^{c}$ be the line of $\mathfrak{l} / \mathfrak{j}$ sent to $\mathcal{E}^{c}\left(x_{0}\right)$ by the orbital map at $x_{0}$. If $\overline{\operatorname{ad}}(\mathfrak{j})\left(D^{c}\right) \subset D^{c}$, then $\mathfrak{l}=\mathfrak{h}$.

Proof. Let us denote by $L$ the connected subgroup of $\mathbf{G}$ of Lie algebra $\mathfrak{l}$, and by $J^{0}$ the identity component of $J=\operatorname{Stab}_{L}\left(x_{0}\right)$. As $\overline{\operatorname{ad}}(\mathfrak{j})$ preserves $D^{c}, \overline{\operatorname{Ad}}(\exp (\mathfrak{j}))$ preserves $D^{c}$, and the subgroup of elements $j \in J^{0}$ such that $\overline{\operatorname{Ad}}(j)$ preserves $D^{c}$ is thus equal to $J^{0}$ by connexity. The construction made in the second assertion of Lemma 4.3 is thus valid for $L / J^{0}$, and proves the existence of an unique $L$-invariant enhanced Lagrangian contact structure $\mathcal{S}_{Y}^{\prime}$ extending $\left(\mathcal{E}^{\alpha}\left(x_{0}\right), \mathcal{E}^{\beta}\left(x_{0}\right), \mathcal{E}^{c}\left(x_{0}\right)\right)$ in the neighbourhood of $x_{0}$. As $\mathfrak{h} \subset \mathfrak{l}$, $H \subset L$, and $\mathcal{S}_{Y}^{\prime}$ is thus $H$-invariant, implying $\mathcal{S}_{Y}^{\prime}=\mathcal{S}_{Y}$ by the unicity of such a tructure. Therefore $\mathfrak{l} \subset \mathfrak{k i r l}_{\mathcal{S}_{Y}}^{\text {loc }}\left(x_{0}\right)=\mathfrak{h}$, which concludes the proof.

Lemma 5.4. Let us assume that $\mathfrak{i}$ is one-dimensional, and let $v$ be a non-zero element of $\mathfrak{i}$. Then the eigenvalues of $\overline{\operatorname{ad}}(v) \in \mathrm{L}(\mathfrak{h} / \mathfrak{i})$ with respect to the eigenlines $D^{\alpha}$ and $D^{\beta}$ are non-zero.

Proof. We already know that $\overline{\mathrm{ad}}(\mathfrak{i})$ is diagonalizable with eigenlines $D^{\alpha}, D^{\beta}$, and $D^{c}$ (see Lemma 4.3). The proof is the same for the eigenvalues of both eigenlines $D^{\alpha}$ and $D^{\beta}$, and we only do it for $D^{\alpha}$. By density of $\operatorname{Rec}(f) \cap \operatorname{Rec}\left(f^{-1}\right)$ in $M$ (see the introduction of Section 3), there exists $x \in O$ such that $\bar{x}=\pi_{M}(x) \in \operatorname{Rec}(f) \cap \operatorname{Rec}\left(f^{-1}\right)$, and possibly replacing $f$ by $f^{-1}$, we have $\lim _{n \rightarrow+\infty}\left\|\left.\mathrm{D}_{\bar{x}} f^{n}\right|_{E^{\alpha}(\bar{x})}\right\|_{M}=0$ for a given Riemannian metric that we fix on $M$.

By hypothesis on $\bar{x}$, there exists a sequence $\left(\gamma_{k}\right)$ in $\pi_{1}(M)$ and a strictly increasing sequence $\left(n_{k}\right)$ of integers such that $\gamma_{k} \tilde{f}^{n_{k}}(x)$ converges to $x$, and we can moreover assume up to extraction that $x_{k} \in O$ for any $k$, implying that $\gamma_{k} \tilde{f}^{n_{k}}$ preserves $O$. Endowing $\tilde{M}$ with the pullback $\tilde{\mu}_{M}$ of the Riemannian metric of $M$, we have $\lim _{k \rightarrow+\infty}\left\|\left.\mathrm{D}_{x}\left(\gamma_{k} \tilde{f}^{n_{k}}\right)\right|_{\tilde{E}^{\alpha}(x)}\right\|_{\tilde{\mu}_{M}}=0$ (since $\pi_{1}(M)$ acts by isometries).

Liouville's theorem 2.9 for the homogeneous model space $\left(\mathbf{X}, \mathcal{L}_{\mathbf{X}}\right)$ implies the existence of a unique sequence $\left(g_{k}\right)$ in $\mathbf{G}$ satisfying

$$
\delta \circ \gamma_{k} \tilde{f}^{n_{k}}=g_{k} \circ \delta \text { on a neighbourhood of } x .
$$

Denoting $x_{0}=\delta(x), g_{k} \cdot x_{0}=\delta \circ \gamma_{k} \tilde{f}^{n_{k}}(x) \in Y=H \cdot x_{0}$ converges to $x_{0}$, and there exists thus a sequence $h_{k} \in H$ converging to the identity in $\mathbf{G}$ and such that $h_{k} \cdot x_{0}=g_{k} \cdot x_{0}$. Since $\delta$ is a local isomorphism from $\left.\tilde{\mathcal{S}}\right|_{O}$ to $\mathcal{S}_{Y}$ on a neighbourhood of $x$, the equation (5.7) defining $g_{k}$ shows that $g_{k}$ preserves $\mathcal{S}_{Y}$ on a neighbourhood of $x_{0}$. By $H$-invariance of $\mathcal{S}_{Y}, i_{k}=h_{k}^{-1} g_{k}$ also preserves $\mathcal{S}_{Y}$, and $i_{k}$ is thus contained in the closed subgroup

$$
I:=\left\{i \in \operatorname{Stab}_{\mathbf{G}}\left(x_{0}\right) \mid i \text { preserves } \mathcal{S}_{Y} \text { on a neighbourhood of } x_{0}\right\}
$$

of $\mathbf{G}$. The Lie algebra of $I$ is equal to $\mathfrak{i}$ because $\mathfrak{i s}_{\mathcal{S}_{Y}}^{\text {loc }}\left(x_{0}\right)=\mathfrak{i}$ (see Lemma 4.3). 
Fact. $I=\left\{i \in \operatorname{Stab}_{\mathbf{G}}\left(x_{0}\right) \mid \operatorname{Ad}(i) \cdot \mathfrak{h}=\mathfrak{h}\right.$ and $\left.\overline{\operatorname{Ad}}(i) \cdot D^{c}=D^{c}\right\}$. In particular $I$ is algebraic and has a finite number of connected components.

Proof. For $i \in I$ and $v \in \mathfrak{h}$, the relation $\mathrm{D}_{x_{0}} i \circ \mathrm{D}_{e} \theta_{x_{0}}=\mathrm{D}_{e} \theta_{x_{0}} \circ \operatorname{Ad}(i)$ implies $i^{-1^{*}} v^{\dagger}=$ $(\operatorname{Ad}(i) \cdot v)^{\dagger}$. Since $i$ is a local automorphism of $\mathcal{S}_{Y}$ and $v^{\dagger}$ a Killing field of $\mathcal{S}_{Y},(\operatorname{Ad}(i) \cdot v)^{\dagger}$ is also a Killing field of $\mathcal{S}_{Y}$, implying $\operatorname{Ad}(i) \cdot v \in \mathfrak{h}$ since $\mathfrak{k i r l}_{\mathcal{S}_{Y}}^{\text {loc }}\left(x_{0}\right)=\mathfrak{h}^{\dagger}$ (see Lemma 4.3). Moreover, $\mathrm{D}_{x_{0}} i\left(\mathcal{E}_{x_{0}}^{c}\right)=\mathcal{E}_{x_{0}}^{c}$ implies $\overline{\operatorname{Ad}}(i) \cdot D^{c}=D^{c}$.

Let us conversely assume that $i \in \operatorname{Stab}_{\mathbf{G}}\left(x_{0}\right)$ satisfies $\operatorname{Ad}(i) \cdot \mathfrak{h}=\mathfrak{h}$ and $\overline{\operatorname{Ad}}(i)$. $D^{c}=D^{c}$. We consider $v \in \mathfrak{h}$ sufficiently close to 0 , such that with $h=\mathrm{e}^{v} \in H$ and $y=h \cdot x_{0} \in Y, \mathcal{S}_{Y}$ is defined at $y$. Since $\overline{\operatorname{Ad}}(i) \cdot D^{c}=D^{c}, \mathrm{D}_{x_{0}} L_{i}\left(\mathcal{E}^{c}\left(x_{0}\right)\right)=\mathcal{E}^{c}\left(x_{0}\right)$, and $h^{\prime}:=i h i^{-1}=\mathrm{e}^{\operatorname{Ad}(i) \cdot v} \in H$ because $\operatorname{Ad}(i) \cdot \mathfrak{h}=\mathfrak{h}$. By $H$-invariance of $\mathcal{E}^{c}$, we obtain $\mathrm{D}_{y} i\left(\mathcal{E}^{c}(y)\right)=\mathrm{D}_{x_{0}} h^{\prime} \circ \mathrm{D}_{x_{0}} i\left(\mathcal{E}^{c}\left(x_{0}\right)\right)=\mathcal{E}^{c}(i \cdot y)$, proving that $i \in I$.

We can thus assume up to extraction that $\left(i_{k}\right)$ lies in a given connected component of $I$, and there exists then $g \in I$ such that $j_{k}=g i_{k}$ is contained in the identity component $I^{0}$. We endow $\mathbf{X}$ with a Riemannian metric $\mu_{\mathbf{X}}$, and denote by $\tilde{\mu}_{\mathbf{X}}=\delta^{*} \mu_{\mathbf{X}}$ its pullback on $\tilde{M}$. Since $\left(\gamma_{k} \tilde{f}^{n_{k}}(x)\right)$ is relatively compact in $\tilde{M}$, the metrics $\tilde{\mu}_{M}$ and $\tilde{\mu}_{\mathbf{X}}$ are equivalent in restriction to $\left(\gamma_{k} \tilde{f}^{n_{k}}(x)\right)$, and the limit stated above for $\tilde{\mu}_{M}$ is thus valid for $\tilde{\mu}_{\mathbf{X}}$, implying $\lim \left\|\left.\mathrm{D}_{x_{0}} g_{k}\right|_{\mathcal{E}^{\alpha}\left(x_{0}\right)}\right\|_{\mu_{\mathbf{X}}}=0$. As $j_{k}=g h_{k}^{-1} g_{k}$ with $\left(g h_{k}^{-1}\right)$ relatively compact in $\mathbf{G}$, we also have $\lim \left\|\left.\mathrm{D}_{x_{0}} j_{k}\right|_{\mathcal{E}^{\alpha}\left(x_{0}\right)}\right\|_{\mu_{\mathbf{X}}}=0$.

$I^{0}$ being connected and one-dimensional, there exists a non-zero $v \in \mathfrak{i}$ and a sequence $t_{k} \in \mathbb{R}$ such that $i_{k}=\exp \left(t_{k} v\right)$, implying that $\mathrm{D}_{x_{0}} j_{k}$ is conjugated by the orbital map to $\exp \left(t_{k} \overline{\operatorname{ad}}(v)\right)$, and thus $\lim \left\|\left.\exp \left(t_{k} \overline{\operatorname{ad}}(v)\right)\right|_{D^{\alpha}}\right\|=0$. Denoting by $\lambda_{\alpha}$ the eigenvalue of $\overline{\mathrm{ad}}(v)$ with respect to $D^{\alpha},\left.\exp \left(t_{k} \overline{\operatorname{ad}}(v)\right)\right|_{D^{\alpha}}=\exp \left(\lambda_{\alpha} t_{k}\right) \mathrm{id}_{D^{\alpha}}$ implies then $\lambda_{\alpha} \neq 0$.

5.3. End of the classification. We now put into our analysis the geometrical and dynamical properties of $\mathfrak{h}$ proved above.

Lemma 5.5. $\mathfrak{h}_{1}=\mathbb{R}^{2} \rtimes \mathfrak{s l}_{2}$ does not satisfy the geometrical conditions of Lemma 4.3.

Proof. The only open orbit of the connected Lie subgroup $H_{1}$ of $\mathbf{G}$ of Lie algebra $\mathfrak{h}_{1}$ is the open subset $\Omega_{a}$ defined in Paragraph 4.2.1. If $H_{1} \cdot x_{0}$ is open for some point $x_{0} \in \mathbf{X}$, we can thus assume that $x_{0}=\left(\left[e_{3}\right],\left[e_{3}, e_{1}\right]\right) \in \Omega_{a}$ up to conjugacy in $H_{1}$, implying that $\mathfrak{i}_{1}=\operatorname{Lie}\left(\operatorname{Stab}_{H_{1}}\left(x_{0}\right)\right)=\left\{\left(\begin{array}{cc}a & b \\ 0 & -a\end{array}\right) \mid a, b \in \mathbb{R}^{2}\right\}$. Denoting $v_{\alpha}=\left(\begin{array}{ll}0 & 0 \\ 1 & 0\end{array}\right)$ and $v_{\beta}=\left(\begin{array}{l}1 \\ 0\end{array}\right) \in \mathfrak{h}_{1}$, we have $\mathbb{R} v_{\alpha}^{\dagger}\left(x_{0}\right)=\mathcal{E}^{\alpha}\left(x_{0}\right)$ and $\mathbb{R} v_{\beta}^{\dagger}\left(x_{0}\right)=\mathcal{E}^{\beta}\left(x_{0}\right)$, and defining $v_{c}=\left(\begin{array}{l}0 \\ 1\end{array}\right)$ and $i=\left(\begin{array}{ll}0 & 1 \\ 0 & 0\end{array}\right) \in \mathfrak{i}_{1}$, the matrix of $\overline{\operatorname{ad}}(i)$ in the basis $\left(\bar{v}_{\alpha}, \bar{v}_{\beta}, \bar{v}_{c}\right)$ of $\mathfrak{h}_{1} / \mathfrak{i}_{1}$ is

$$
\operatorname{Mat}_{\left(\bar{v}_{\alpha}, \bar{v}_{\beta}, \bar{v}_{c}\right)} \overline{\operatorname{ad}}(i)=\left(\begin{array}{lll}
0 & 0 & 0 \\
0 & 0 & 1 \\
0 & 0 & 0
\end{array}\right) .
$$

Any line of $\mathfrak{h}_{1} / \mathfrak{i}_{1}$ that is transverse to $\operatorname{Vect}\left(\bar{v}_{\alpha}, \bar{v}_{\beta}\right)$ has projective coordinates $[a, b, 1]$ in the basis $\left(\bar{v}_{\alpha}, \bar{v}_{\beta}, \bar{v}_{c}\right)$ for some $(a, b) \in \mathbb{R}^{2}$, and $\overline{\operatorname{ad}}(i)\left(D^{c}\right)$ has thus coordinates $[0,1,0]$. This proves that $\overline{\operatorname{ad}}(i)\left(D^{c}\right) \not \subset D^{c}$, i.e. that $\mathfrak{h}_{1}$ does not satisfy the geometrical conditions of Lemma 4.3.

Lemma 5.6. If $\mathfrak{h}$ is a four-dimensional subalgebra of $\mathfrak{h}_{\mathbf{a}}=\mathfrak{p}_{\text {min }}$, or is equal to $\mathfrak{h}_{2}=$ $\mathbb{R}^{2} \rtimes \mathfrak{s i m}\left(\mathbb{R}^{2}\right)$, then $\mathfrak{h}$ does not respect both the geometrical conditions of Lemma 4.3 and the dynamical condition of Lemma 5.4. 
Proof. We first assume that $\mathfrak{h}$ is a four-dimensional subalgebra of $\mathfrak{p}_{\text {min }}$. Therefore $H \subset$ $\mathbf{P}_{\text {min }}$, and if $H \cdot x_{0}$ is open then $x_{0} \in Y_{\mathbf{a}}$ according to Proposition 4.5. We can thus assume up to conjugacy in $H$ that $x_{0}=o_{\mathbf{a}}=\left(\left[e_{3}\right],\left[e_{3}, e_{2}\right]\right) \in Y_{\mathbf{a}}$, implying that

$$
\mathfrak{i}=\mathfrak{s t a b} \mathfrak{b}_{\mathfrak{h}}\left(o_{\mathbf{a}}\right) \subset \mathfrak{i}_{\mathbf{a}}=\mathfrak{s t a b} \mathfrak{b}_{\mathfrak{p}_{\text {min }}}\left(o_{\mathbf{a}}\right)=\left\{\left(\begin{array}{ccc}
a & 0 & 0 \\
0 & -a-b & 0 \\
0 & 0 & b
\end{array}\right) \mid(a, b) \in \mathbb{R}^{2}\right\} .
$$

Let $D^{c} \subset \mathfrak{h} / \mathfrak{i}$ be a line preserved by $\overline{\mathrm{ad}}(\mathfrak{i})$, and such that $\mathrm{D}_{e} \bar{\theta}_{o_{\mathbf{a}}}\left(D^{c}\right)$ is transverse to $\left(\mathcal{E}^{\alpha} \oplus\right.$ $\left.\mathcal{E}^{\beta}\right)\left(o_{\mathbf{a}}\right)$. Since $\mathfrak{h}$ is a proper subalgebra of $\mathfrak{p}_{\text {min }}$, Lemma 5.3 implies that $\overline{\operatorname{ad}}\left(\mathfrak{i}_{\mathbf{a}}\right)\left(D^{c}\right) \not \subset D^{c}$, and thus that $\mathfrak{s t a b}_{\mathfrak{i}_{\mathbf{a}}}\left(D^{c}\right)=\left\{v \in \mathfrak{i}_{\mathbf{a}} \mid \overline{\operatorname{ad}}(v)\left(D^{c}\right) \subset D^{c}\right\}=\mathfrak{i}$ is one-dimensional. Any line $D^{c}$ of $\mathfrak{p}_{\text {min }} / \mathfrak{i}_{\mathbf{a}}$ which is transverse to the contact plane has projective coordinates $[x, y, 1]$ in the basis $(\bar{X}, \bar{Y}, \bar{Z})$ of $\mathfrak{p}_{\text {min }} / \mathfrak{i}_{\mathbf{a}}$, for some $(x, y) \in \mathbb{R}^{2}$ (see Proposition 4.6 ), and according to (4.16), we have:

- if $x=y=0$, i.e. $D^{c}=\mathbb{R} \bar{Z}$, then $\mathfrak{s t a b} \mathfrak{b}_{\mathfrak{i}_{\mathbf{a}}}(\mathbb{R} \bar{Z})=\mathfrak{i}_{\mathbf{a}}$;

- if $x=0$ and $y \neq 0$, i.e. $D^{c}=D_{Y}^{c}(t):=\mathbb{R}(\bar{Z}+t \bar{Y})$ for some $t \in \mathbb{R}$, then $\mathfrak{s t a b} \mathfrak{i}_{\mathfrak{i}_{\mathrm{a}}}\left(D_{Y}^{c}(t)\right)$ is equal to the line $\mathfrak{i}_{Y}$ generated by the diagonal matrix $[1,1,-2]=$ $\left(\begin{array}{ccc}1 & 0 & 0 \\ 0 & 1 & 0 \\ 0 & 0 & -2\end{array}\right)$;

- if $x \neq 0$ and $y=0$, i.e. $D^{c}=D_{X}^{c}(t):=\mathbb{R}(\bar{Z}+t \bar{X})$ for some $t \in \mathbb{R}$, then $\mathfrak{s t a b} \mathfrak{b}_{\mathfrak{i}_{\mathbf{a}}}\left(D_{X}^{c}(t)\right)$ is equal to the line $\mathfrak{i}_{X}$ generated by the diagonal matrix $[-2,1,1]=$ $\left(\begin{array}{ccc}-2 & 0 & 0 \\ 0 & 1 & 0 \\ 0 & 0 & 1\end{array}\right)$;

- if $x \neq 0$ and $y \neq 0$, then $\mathfrak{s t a b} \mathfrak{b}_{\mathfrak{a}}\left(D^{c}\right)=\{0\}$.

The only transverse lines with a one-dimensional stabilizer being $D_{X}^{c}(t)$ and $D_{Y}^{c}(t), \mathfrak{i}$ is

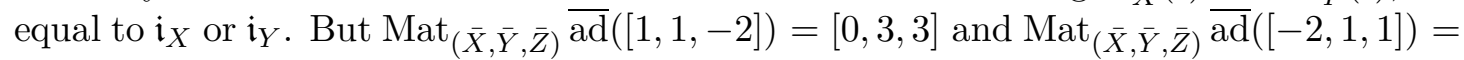
$[-3,0,-3]$ according to $(4.16)$, i.e. the elements of $\mathfrak{i}_{X}$ and $\mathfrak{i}_{Y}$ have zero eigenvalue with respect to either the $\alpha$ or the $\beta$-direction, proving that $\mathfrak{h}$ does not satisfy the dynamical condition of Lemma 5.4.

In the same way, if $\mathfrak{h}=\mathfrak{h}_{2}$, then we can assume that $x_{0}=o_{\mathbf{a}} \in \Omega_{a}$ up to conjugacy in $H_{2}=\mathbb{R}^{2} \rtimes \operatorname{Sim}\left(\mathbb{R}^{2}\right)$, implying $\mathfrak{i}_{2}=\mathfrak{s} \mathfrak{t a} \mathfrak{b}_{\mathfrak{h}_{2}}\left(o_{\mathbf{a}}\right)=\mathfrak{i}_{Y}$ defined above. We saw that the elements of $\mathfrak{i}_{Y}$ have zero eigenvalue with respect to the $\alpha$-direction, proving that $\mathfrak{h}_{2}$ does not satisfy the dynamical condition of Lemma 5.4.

Proposition 4.4 now directly follows from Lemmas 5.2, 5.5 and 5.6.

\section{Global structure}

From the local model that we determined for the enhanced Lagrangian contact structure $\mathcal{S}$, we will now deduce a global information.

6.1. Local homogeneity of the enhanced Lagrangian contact structure. So far, we only have informations on a dense and open subset $\Omega$ of $M$ (see Propositions 3.2 and 4.6), and the first step to obtain a global information is to prove the following.

Proposition 6.1. The open dense subset $\Omega$ equals $M$, i.e. $\mathcal{S}$ is locally homogeneous on $M$.

We will denote in this paragraph by $(\mathcal{C}, \varphi)=(\hat{M}, \omega, \varphi)$ the normal generalized Car$\tan$ geometry of the enhanced Lagrangian contact structure $\tilde{\mathcal{S}}=\pi_{M}^{*} \mathcal{S}$ of $\tilde{M}$, and by 
$\mathcal{K}^{\text {tot }}: \hat{M} \rightarrow W_{\mathcal{K}^{t o t}}$ its total curvature (see Paragraphs 2.3.4 and 2.4.2). We recall that $\tilde{\Omega}=\pi_{M}^{-1}(\Omega) \subset \tilde{M}$, and that the projection of the Cartan bundle is denoted by $\pi: \hat{M} \rightarrow \tilde{M}$.

We also recall that the local homogeneity of $\left.\tilde{\mathcal{S}}\right|_{\tilde{\Omega}}$ means that the connected components of $\tilde{\Omega}$ are exactly its Kill ${ }^{l o c}$-orbits (see Definition 3.1). Since the rank of D $\mathcal{K}^{\text {tot }}$ is invariant by the right action of $\mathbf{P}_{\text {min }}$ and by the flow of Killing fields, this shows that $\operatorname{rk}\left(\mathrm{D} \mathcal{K}^{\text {tot }}\right)$ is constant over any connected component of $\tilde{\Omega}$.

We choose for this whole paragraph a connected component $O$ of $\tilde{\Omega}$ (i.e. a Kill ${ }^{l o c}$-orbit of $\tilde{\mathcal{S}})$ such that $\operatorname{rk}\left(\mathrm{D}_{\hat{x}} \mathcal{K}^{t o t}\right)$ for $\hat{x} \in \pi^{-1}(O)$ is maximal among $\operatorname{rk}\left(\mathrm{D}_{\hat{x}} \mathcal{K}^{t o t}\right)$ for $\hat{x} \in \pi^{-1}(\tilde{\Omega})$. We will denote by $\left(Y, \mathcal{S}_{Y}\right)$ the local model of $\left.\tilde{\mathcal{S}}\right|_{O}$, equal to $\left(Y_{\mathbf{t}}, \mathcal{S}_{\mathbf{t}}\right)$ or $\left(Y_{\mathbf{a}}, \mathcal{S}_{\mathbf{a}}\right)$ and such that $\left.\delta\right|_{O}:\left(O,\left.\tilde{\mathcal{S}}\right|_{O}\right) \rightarrow\left(Y, \mathcal{S}_{Y}\right)$ is a local isomorphism (see Corollary 4.6). We still denote by $\mathfrak{h}$ the subalgebra of Killing fields of $\mathcal{S}_{Y}$, respectively equal to $\mathfrak{h}_{\mathbf{t}}$ or $\mathfrak{h}_{\mathbf{a}}$ (see Proposition 4.4), and by $H$ the corresponding Lie connected subgroup

$$
H_{\mathbf{t}}^{0}=\left[\begin{array}{cc}
\mathrm{GL}_{2}^{+}(\mathbb{R}) & 0 \\
0 & 1
\end{array}\right] \text { or } H_{\mathbf{a}}^{0}=\mathbf{P}_{m i n}^{+}
$$

of $\mathbf{G}$ of Lie algebra $\mathfrak{h}$, preserving $\mathcal{S}_{Y}$.

We recall that $\delta: \tilde{M} \rightarrow \mathbf{X}$ denotes the developping map of the $(\mathbf{G}, \mathbf{X})$-structure of $M$ describing the flat Lagrangian contact structure $\mathcal{L}$ (see Proposition 3.4).

Lemma 6.2. The boundary of $O$ is mapped to $\mathbf{X} \backslash Y$ by the developping map: $\delta(\partial O) \subset$ $\mathbf{X} \backslash Y$.

Proof. Let us assume by contradiction that there exists $x \in \partial O$ such that $x_{0}=\delta(x) \in Y$. The pullback $\tilde{\mathfrak{h}}:=\delta^{*} \mathfrak{h}=\left\{\delta^{*} v \mid v \in \mathfrak{h}\right\}$ is a subalgebra of vector fields on $\tilde{M}$, such that $\mathfrak{K i f l}\left(O,\left.\tilde{\mathcal{S}}\right|_{O}\right)=\left.\tilde{\mathfrak{h}}\right|_{O}$ according to Lemma 4.1. As $x_{0} \in Y$, there exists an open and convex neighbourhood $W_{0}$ of 0 in $\mathfrak{h}$ such that $V=\exp \left(W_{0}\right) \cdot x_{0} \subset Y$ is an open neighbourhood of $x_{0}$. Denoting $W=\delta^{*} W_{0} \subset \tilde{\mathfrak{h}}, U=\left\{\varphi_{X}^{1}(x) \mid X \in W\right\}$ is thus an open neighbourhood of $x$, and possibly shrinking $W_{0}$, we can moreover assume that $\left.\delta\right|_{U}$ is a diffeomorphism from $U$ to $V$. As $x \in \partial O$, there exists $y \in U \cap O$, and $X \in W$ such that $x=\varphi_{X}^{1}(y)$, implying that $\varphi_{X}^{t}(y) \in U$ for any $t \in[0 ; 1]$, and thus $\delta\left(\varphi_{X}^{t}(y)\right) \in V \subset Y$. Denoting $t_{0}=\inf \left\{t \in[0 ; 1] \mid \varphi_{X}^{t}(y) \in \partial O\right\}, t_{0}>0$ because $O$ is open, and $\varphi_{X}^{t_{0}}(y) \in \partial O$ because $\partial O$ is closed. Replacing $x$ by $\varphi_{X}^{t_{0}}(y)$ and $X$ by $\frac{X}{t_{0}} \in W$, we finally have $y \in O$, $x=\varphi_{X}^{1}(y) \in \partial O$, and for any $t \in\left[0 ; 1\left[, \varphi_{X}^{t}(y) \in O\right.\right.$, with $\left.X\right|_{O} \in \operatorname{Kill}\left(O,\left.\tilde{\mathcal{S}}\right|_{O}\right)$.

Choosing $\hat{y} \in \pi^{-1}(y)$, the invariance of $D^{1} \mathcal{K}^{\text {tot }}$ by local automorphisms and the fact that $\varphi_{X}^{t}$ is a local automorphism of $(\mathcal{C}, \varphi)$ on the neighbourhood of $y$ for any $t \in[0 ; 1[$ implies $D^{1} \mathcal{K}^{t o t}\left(\hat{\varphi}_{X}^{t}(\hat{y})\right)=D^{1} \mathcal{K}^{t o t}(\hat{y})$ for any $t \in\left[0 ; 1\left[\right.\right.$. Denoting $\hat{x}=\hat{\varphi}_{X}^{1}(\hat{y})$, we obtain $D^{1} \mathcal{K}^{t o t}(\hat{x})=D^{1} \mathcal{K}^{t o t}(\hat{y})$ by continuity, i.e. $\mathcal{K}^{\text {tot }}(\hat{x})=\mathcal{K}^{t o t}(\hat{y})$ and $\mathrm{D}_{\hat{x}} \mathcal{K}^{t o t} \circ \omega_{\hat{x}}^{-1}=\mathrm{D}_{\hat{y}} \mathcal{K}^{t o t} \circ$ $\omega_{\hat{y}}^{-1}$.

This implies $\hat{x} \in \hat{M}^{i n t}$. In fact as the rank of $\mathrm{D} \mathcal{K}^{\text {tot }}$ can only increase locally, there is an open neighbourhood $\mathcal{U}$ of $\hat{x}$ where the rank of $\mathrm{D}^{\text {tot }}$ is greater than $\operatorname{rk}\left(\mathrm{D}_{\hat{x}} \mathcal{K}^{t o t}\right)$. Let us assume by contradiction that the open subset of $\mathcal{U}$ where $\operatorname{rk}\left(\mathrm{D}_{\hat{x}^{\prime}} \mathcal{K}^{\text {tot }}\right)>\operatorname{rk}\left(\mathrm{D}_{\hat{x}} \mathcal{K}^{\text {tot }}\right)$ is non-empty. Then by density of $\pi^{-1}(\tilde{\Omega})$, there exists $\hat{z} \in \pi^{-1}(\tilde{\Omega})$ such that $\operatorname{rk}\left(\mathrm{D}_{\hat{z}} \mathcal{K}^{\text {tot }}\right)>$ $\operatorname{rk}\left(\mathrm{D}_{\hat{x}} \mathcal{K}^{t o t}\right)$. But $\operatorname{rk}\left(\mathrm{D}_{\hat{x}} \mathcal{K}^{t o t}\right)=\operatorname{rk}\left(\mathrm{D}_{\hat{y}} \mathcal{K}^{\text {tot }}\right)$ because $D^{1} \mathcal{K}^{\text {tot }}(\hat{x})=D^{1} \mathcal{K}^{\text {tot }}(\hat{y})$, and thus 
$\operatorname{rk}\left(\mathrm{D}_{\hat{z}} \mathcal{K}^{t o t}\right)>\operatorname{rk}\left(\mathrm{D}_{\hat{y}} \mathcal{K}^{t o t}\right)$ with $\hat{y} \in \pi^{-1}(O)$, wich contradicts our hypothesis of maximality of $\operatorname{rk}\left(\mathrm{D} \mathcal{K}^{t o t}\right)$ on $O$. Therefore $\operatorname{rk}\left(\mathrm{D} \mathcal{K}^{t o t}\right)$ is constant on the open neighbourhood $\mathcal{U}$ of $\hat{x}$, proving that $\hat{x} \in \hat{M}^{i n t}$ according to Integrability theorem 2.17 .

As the Kill ${ }^{l o c}$-orbit $O$ of $y$ is open, $\omega_{\hat{y}}^{-1}\left(\mathfrak{p}_{\text {min }}\right)+\operatorname{Ker}\left(\mathrm{D}_{\hat{y}} \mathcal{K}^{t o t}\right)=\mathrm{T}_{\hat{y}} \hat{M}$, and therefore $\omega_{\hat{x}}^{-1}\left(\mathfrak{p}_{\text {min }}\right)+\operatorname{Ker}\left(\mathrm{D}_{\hat{x}} \mathcal{K}^{t o t}\right)=\mathrm{T}_{\hat{x}} \hat{M}$ because $\mathrm{D}_{\hat{x}} \mathcal{K}^{t o t} \circ \omega_{\hat{x}}^{-1}=\mathrm{D}_{\hat{y}} \mathcal{K}^{t o t} \circ \omega_{\hat{y}}^{-1}$. Since $\hat{x} \in \hat{M}^{i n t}$, for any $v \in \operatorname{Ker}\left(\mathrm{D}_{\hat{x}} \mathcal{K}^{\text {tot }}\right)$ there is a local Killing field $X$ of $\tilde{\mathcal{S}}$ defined in the neighbourhood of $x$ such that $\hat{X}_{\hat{x}}=v$. But $\omega_{\hat{x}}^{-1}\left(\mathfrak{p}_{\text {min }}\right)+\operatorname{Ker}\left(\mathrm{D}_{\hat{x}} \mathcal{K}^{t o t}\right)=\mathrm{T}_{\hat{x}} \hat{M}$, and we thus have $\left\{X_{x} \mid X \in \mathfrak{k i l l}_{\tilde{\mathcal{S}}}^{l o c}(x)\right\}=\mathrm{T}_{x} \tilde{M}$, implying that the Kill ${ }^{l o c}$-orbit of $x$ is open. Since $x \in \partial O$, the Kill ${ }^{l o c}$-orbit of $x$ intersects thus the Kill ${ }^{l o c}$-orbit $O$, i.e. $x \in O$, which contradicts our initial hypothesis. This contradiction concludes the proof of the lemma.

Lemma 6.2 allows us to reduce the study of the central direction $\tilde{E}^{c}$ on the boundary of $O$, to the study of the central direction $\mathcal{E}^{c}$ on the boundary of $Y$. We first do some geometrical remarks about the open subsets $Y_{\mathbf{a}}$ and $Y_{\mathbf{t}}$ of $\mathbf{X}$, defined in Paragraphs 4.2.2 and 4.2.3.

Let us recall that, denoting $D_{\infty}=\left[e_{1}, e_{2}\right], m_{\mathbf{t}}=\left[e_{3}\right]$ and $m_{\mathbf{a}}=\left[e_{1}\right]$, we have

$$
Y_{\mathbf{t}}=\mathbf{X} \backslash\left(S_{\beta, \alpha}\left(D_{\infty}\right) \cup S_{\alpha, \beta}\left(m_{\mathbf{t}}\right)\right) \text { and } Y_{\mathbf{a}}=\mathbf{X} \backslash\left(S_{\beta, \alpha}\left(D_{\infty}\right) \cup S_{\beta, \alpha}\left(m_{\mathbf{a}}\right)\right) .
$$

In particular, for $\varepsilon=\mathbf{a}$ and $\mathbf{t}: \mathbf{X} \backslash Y_{\varepsilon}=\partial Y_{\varepsilon}=S_{\beta, \alpha}\left(D_{\infty}\right) \cup S_{\alpha, \beta}\left(m_{\varepsilon}\right)$.

We define in both cases

$$
\mathcal{G}:=\left\{x \in \partial Y \mid \mathcal{C}^{\alpha}(x) \nsubseteq \partial Y \text { or } \mathcal{C}^{\beta}(x) \nsubseteq \subseteq \partial Y\right\}
$$

It is easy to check that for $\varepsilon=\mathbf{a}$ and $\mathbf{t}$, we have

$$
\mathcal{G}_{\varepsilon}=\partial Y_{\varepsilon} \backslash\left\{\mathcal{C}^{\beta}\left(D_{\infty}\right) \cup \mathcal{C}^{\alpha}\left(m_{\varepsilon}\right) \cup\left(S_{\beta, \alpha}\left(D_{\infty}\right) \cap S_{\alpha, \beta}\left(m_{\varepsilon}\right)\right)\right\},
$$

and that for any $x \in \mathcal{G}$, if $\mathcal{C}^{\varepsilon}(x) \nsubseteq \partial Y$ for $\varepsilon=\alpha$ or $\beta$, then $\mathcal{C}^{\varepsilon}(x) \backslash\{x\} \subset Y$.

We have $S_{\beta, \alpha}\left(D_{\infty}\right) \cap S_{\alpha, \beta}\left(m_{\mathbf{a}}\right)=\mathcal{C}^{\beta}\left(D_{\infty}\right) \cup \mathcal{C}^{\alpha}\left(m_{\mathbf{a}}\right)$, and $S_{\beta, \alpha}\left(D_{\infty}\right) \cap S_{\alpha, \beta}\left(m_{\mathbf{t}}\right)$ is equal to the chain defined by $\left(m_{\mathbf{t}}, D_{\infty}\right)$, denoted by $\mathcal{C}\left(m_{\mathbf{t}}, D_{\infty}\right)$ and defined as follows:

$$
\mathcal{C}\left(m_{\mathbf{t}}, D_{\infty}\right):=\left\{\left(m,\left[m, m_{\mathbf{t}}\right]\right) \mid m \in D_{\infty}\right\} .
$$

Finally, we will use the following description of the respective orbits of $H$ on $\mathcal{G}$ :

(1) the orbits of $H_{\mathbf{t}}^{0}$ on $\mathcal{G}_{\mathbf{t}}$ are $\mathcal{G}_{\mathbf{t}}^{1}=S_{\alpha, \beta}\left(m_{\mathbf{t}}\right) \backslash\left(\mathcal{C}^{\alpha}\left(m_{\mathbf{t}}\right) \cup \mathcal{C}\left(m_{\mathbf{t}}, D_{\infty}\right)\right)$ where $\mathcal{C}^{\alpha}(x) \backslash$ $\{x\} \subset Y_{\mathbf{t}}$, and $\mathcal{G}_{\mathbf{t}}^{2}=S_{\beta, \alpha}\left(D_{\infty}\right) \backslash\left(\mathcal{C}^{\beta}\left(D_{\infty}\right) \cup \mathcal{C}\left(m_{\mathbf{t}}, D_{\infty}\right)\right)$ where $\mathcal{C}^{\beta}(x) \backslash\{x\} \subset Y_{\mathbf{t}} ;$

(2) the orbits of $H_{\mathbf{a}}^{0}$ on $\mathcal{G}_{\mathbf{a}}$ are $\mathcal{G}_{\mathbf{a}}^{1}=S_{\alpha, \beta}\left(m_{\mathbf{a}}\right) \backslash\left(\mathcal{C}^{\alpha}\left(m_{\mathbf{a}}\right) \cup \mathcal{C}^{\beta}\left(D_{\infty}\right)\right)$ where $\mathcal{C}^{\alpha}(x) \backslash$ $\{x\} \subset Y_{\mathbf{a}}$ and $\mathcal{G}_{\mathbf{a}}^{2}=S_{\beta, \alpha}\left(D_{\infty}\right) \backslash\left(\mathcal{C}^{\alpha}\left(m_{\mathbf{a}}\right) \cup \mathcal{C}^{\beta}\left(D_{\infty}\right)\right)$ where $\mathcal{C}^{\beta}(x) \backslash\{x\} \subset Y_{\mathbf{a}}$.

We will now prove that the central direction $\mathcal{E}^{c}$ degenerates along the $\alpha$ and $\beta$-circles when converging to a point of $\mathcal{G}$.

Lemma 6.3. Let $\gamma:[0 ; 1] \rightarrow \mathbf{X}$ be a smooth path such that $\gamma(] 0 ; 1]) \subset Y, x=\gamma(0) \in \mathcal{G}$, and $\gamma([0 ; 1])$ is entirely contained in $\mathcal{C}^{\alpha}(x)$, or entirely contained in $\mathcal{C}^{\beta}(x)$. Then $\mathcal{E}^{c}(\gamma(t))$ converges at $t=0$ to a line contained in $\left(\mathcal{E}^{\alpha} \oplus \mathcal{E}^{\beta}\right)(x)$.

Proof. As the action of $H$ on $Y$ preserves $\mathcal{E}^{c}$, it will be sufficient to prove this result for one point of each of the two orbits of $H$ on $\mathcal{G}$ described above, in each of the two cases $Y_{\mathbf{t}}$ or $Y_{\mathbf{a}}$. We thus have only four cases to handle, and we saw that in each case, either $\mathcal{C}^{\alpha}(x) \backslash\{x\} \subset Y$ and $\mathcal{C}^{\beta}(x) \subset \partial Y$, or the contrary. We thus have only one 
possibility to consider for $\gamma$ in each of these four cases, either that $\gamma([0 ; 1]) \subset \mathcal{C}^{\alpha}(x)$, or that $\gamma([0 ; 1]) \subset \mathcal{C}^{\beta}(x)$. To clarify our strategy, let $x$ be a point of $\mathcal{G}_{\mu}^{i}$ for $\mu=\mathbf{t}$ or $\mathbf{a}$ and $i=1$ or 2 , and let us consider the following data:

- a one-parameter subgroup $\left\{g^{t}\right\}_{t \in \mathbb{R}}$ of $\mathbf{G}$ such that, denoting $x(t)=g^{t} \cdot x$, we have $\{x(t) \mid t \in \mathbb{R}\}=\mathcal{C}^{\varepsilon}(x) \backslash\{y\}$, with $y \in \mathcal{C}^{\varepsilon}(x) \cap Y$, and $\varepsilon=\alpha$ or $\beta$ according to the case considered,

- a one-parameter subgroup $\left\{h^{t}\right\}_{t \in \mathbb{R}}$ of $H$ such that $g^{t} \cdot x=x(t)=h^{t^{-1}} \cdot y$ for any $t \in \mathbb{R}^{*}$,

- $A$ in $\mathfrak{s l}_{3}$ such that $\mathrm{D}_{e} \theta_{y}(\mathbb{R} A)=\mathcal{E}^{c}(y)$, where $\theta_{y}: \mathbf{G} \rightarrow \mathbf{X}$ is the orbital map at $y$,

- and $g_{0} \in \mathbf{G}$ such that $g_{0} \cdot x=o$ where $o=\left(\left[e_{1}\right],\left[e_{1}, e_{2}\right]\right)$ is the usual base-point of $\mathbf{X}$.

Then for any $t \in \mathbb{R}^{*}$ we have $\left.\mathrm{D}_{x(t)}\left(g_{0} g^{-t}\right)\left(\mathcal{E}^{c}(x(t))\right)=\mathrm{D}_{e} \theta_{o}\left(\mathbb{R} \operatorname{Ad}\left(g_{0} g^{-t} h^{t^{-1}}\right) \cdot A\right)\right)$. Denoting by $p: \mathfrak{s l}_{3} \rightarrow \mathfrak{s l}_{3} / \mathfrak{p}_{\text {min }}$ the canonical projection, let us assume that $p\left(\mathbb{R} \operatorname{Ad}\left(g_{0} g^{-t} h^{t^{-1}}\right)\right.$. $A)$ converges at $t=0$ to a line contained in $\operatorname{Vect}\left(\bar{e}_{\alpha}, \bar{e}_{\beta}\right)$. Then $\mathrm{D}_{e} \theta_{o}\left(\mathbb{R} \operatorname{Ad}\left(g_{0} g^{-t} h^{t^{-1}}\right)\right.$. $A) \subset \mathrm{T}_{o} \mathbf{X}$ converges to a line $L \subset\left(\mathcal{E}^{\alpha} \oplus \mathcal{E}^{\beta}\right)(o)$, and as $g^{t} g_{0}^{-1}$ converges to $g_{0}^{-1}$ at $t=0$, we deduce by continuity that $\mathcal{E}^{c}(x(t))$ converges at $t=0$ to $\mathrm{D}_{o} g_{0}^{-1}(L)$, contained in $\left(\mathcal{E}^{\alpha} \oplus \mathcal{E}^{\beta}\right)(x)$, because $g_{0}^{-1}$ preserves $\mathcal{E}^{\alpha} \oplus \mathcal{E}^{\beta}$.

In conclusion, we only have to find, in each of the four cases $\mu=\mathbf{t}$ or $\mathbf{a}$ and $i=1$ or 2 , a point $x \in \mathcal{G}_{\mu}^{i}$, together with $g^{t}, h^{t}, A$, and $g_{0}$ satisfying the above conditions, and to prove that $p\left(\mathbb{R} \operatorname{Ad}\left(g_{0} g^{-t} h^{t^{-1}}\right) \cdot A\right)$ converges at $t=0$ to a line contained in $\operatorname{Vect}\left(\bar{e}_{\alpha}, \bar{e}_{\beta}\right)$.

We begin with $Y_{\mathbf{t}}$, for which we choose for both orbits $\mathcal{G}_{\mathbf{t}}^{1}$ and $\mathcal{G}_{\mathbf{t}}^{2}$ the point $y:=$ $o_{\mathbf{t}}=\left([1,0,1],\left[(1,0,1), e_{2}\right]\right) \in Y_{\mathbf{t}}$. Let us recall that in this case, $A=\left(\begin{array}{ccc}1 & 0 & 0 \\ 0 & -1 & 0 \\ 0 & 0 & 0\end{array}\right)$ satisfies $\mathcal{E}^{c}\left(o_{\mathbf{t}}\right)=\mathrm{D}_{e} \theta_{o_{\mathbf{t}}}(\mathbb{R} A)$ (see Paragraph 4.2.2).

- For $\mathcal{G}_{\mathbf{t}}^{1}$, choosing $x=\left([1,0,1],\left[(1,0,1), e_{1}\right]\right)=\left([1,0,1],\left[e_{1}, e_{3}\right]\right), g_{0}=\left(\begin{array}{ccc}1 & 0 & 0 \\ 1 & 0 & -1 \\ 0 & 1 & 0\end{array}\right)$, and the one-parameter subgroups $g^{t}=\left(\begin{array}{ccc}1 & 0 & 0 \\ t & 1 & -t \\ 0 & 0 & 1\end{array}\right)$ of $\mathbf{G}$ and $h^{t}=\left(\begin{array}{lll}1 & t & 0 \\ 0 & 1 & 0 \\ 0 & 0 & 1\end{array}\right)$ of $H_{\mathbf{t}}^{0}$ such that $g^{t} \cdot x=h^{t^{-1}} \cdot o_{\mathbf{t}} \in \mathcal{C}^{\alpha}(x)$, we obtain:

$$
\operatorname{Ad}\left(g_{0} g^{-t} h^{t^{-1}}\right) \cdot A=\left(\begin{array}{ccc}
1 & -2 & -2 t^{-1} \\
1 & -2 & -2 t^{-1} \\
-t & t & 1
\end{array}\right)
$$

and thus $p\left(\mathbb{R} \operatorname{Ad}\left(g_{0} g^{-t} h^{t^{-1}}\right) \cdot A\right)$ converges at $t=0$ to $\mathbb{R} \bar{e}_{\beta}$.

- For $\mathcal{G}_{\mathbf{t}}^{2}$, choosing $x=\left(\left[e_{2}\right],\left[e_{2},(1,0,1)\right]\right), g_{0}=\left(\begin{array}{ccc}0 & 1 & 0 \\ 1 & 0 & 0 \\ 1 & 0 & -1\end{array}\right)$, and the one-parameter subgroups $g^{t}=\left(\begin{array}{lll}1 & t & 0 \\ 0 & 1 & 0 \\ 0 & t & 1\end{array}\right)$ of $\mathbf{G}$ and $h^{t}=\left(\begin{array}{lll}1 & 0 & 0 \\ t & 1 & 0 \\ 0 & 0 & 1\end{array}\right)$ of $H_{\mathbf{t}}^{0}$ such that $g^{t} \cdot x=h^{t^{-1}} \cdot o_{\mathbf{t}} \in$ $\mathcal{C}^{\beta}(x)$, we obtain

$$
\operatorname{Ad}\left(g_{0} g^{-t} h^{t^{-1}}\right) \cdot A=\left(\begin{array}{ccc}
1 & 2 t^{-1} & 0 \\
0 & -1 & 0 \\
t & 1 & 0
\end{array}\right)
$$

and thus $p\left(\mathbb{R} \operatorname{Ad}\left(g_{0} g^{-t} h^{t^{-1}}\right) \cdot A\right)$ converges at $t=0$ to $\mathbb{R} \bar{e}_{\alpha}$.

We now consider the case of $Y_{\mathbf{a}}$, for which we choose for both orbits $\mathcal{G}_{\mathbf{a}}^{1}$ and $\mathcal{G}_{\mathbf{a}}^{2}$ the point $y:=o_{\mathbf{a}}=\left(\left[e_{3}\right],\left[e_{3}, e_{2}\right]\right) \in Y_{\mathbf{a}}$, and we recall that in this case $A=\left(\begin{array}{lll}0 & 0 & 1 \\ 0 & 0 & 0 \\ 0 & 0 & 0\end{array}\right)$ satisfies the above condition $\mathcal{E}^{c}\left(o_{\mathbf{a}}\right)=\mathrm{D}_{e} \theta_{o_{\mathbf{a}}}(\mathbb{R} A)$ (see Paragraph 4.2.3). 
- For $\mathcal{G}_{\mathbf{a}}^{1}$, choosing $x=\left(\left[e_{3}\right],\left[e_{3}, e_{1}\right]\right), g_{0}=\left(\begin{array}{lll}0 & 0 & 1 \\ 1 & 0 & 0 \\ 0 & 1 & 0\end{array}\right)$, and the one-parameter subgroups $g^{t}=\left(\begin{array}{lll}1 & 0 & 0 \\ t & 1 & 0 \\ 0 & 0 & 1\end{array}\right)$ of $\mathbf{G}$ and $h^{t}=\left(\begin{array}{lll}1 & t & 0 \\ 0 & 1 & 0 \\ 0 & 0 & 1\end{array}\right)$ of $H_{\mathbf{a}}^{0}$ such that $g^{t} \cdot x=h^{t^{-1}} \cdot o_{\mathbf{a}} \in$ $\mathcal{C}^{\alpha}(x)$, we obtain:

$$
\operatorname{Ad}\left(g_{0} g^{-t} h^{t^{-1}}\right) \cdot A=\left(\begin{array}{ccc}
0 & 0 & 0 \\
1 & 0 & 0 \\
-t & 0 & 0
\end{array}\right),
$$

and thus $p\left(\mathbb{R} \operatorname{Ad}\left(g_{0} g^{-t} h^{t^{-1}}\right) \cdot A\right)$ converges at $t=0$ to $\mathbb{R} \bar{e}_{\beta}$.

- For $\mathcal{G}_{\mathbf{a}}^{2}$, choosing $x=\left(\left[e_{2}\right],\left[e_{2}, e_{3}\right]\right), g_{0}=\left(\begin{array}{lll}0 & 1 & 0 \\ 0 & 0 & 1 \\ 1 & 0 & 0\end{array}\right)$, and the one-parameter subgroups $g^{t}=\left(\begin{array}{lll}1 & 0 & 0 \\ 0 & 1 & 0 \\ 0 & t & 1\end{array}\right)$ of $\mathbf{G}$ and $h^{t}=\left(\begin{array}{lll}1 & 0 & 0 \\ 0 & 1 & t \\ 0 & 0 & 1\end{array}\right)$ of $H_{\mathbf{a}}^{0}$ such that $g^{t} \cdot x=h^{t^{-1}} \cdot o_{\mathbf{a}} \in$ $\mathcal{C}^{\beta}(x)$, we obtain

$$
\operatorname{Ad}\left(g_{0} g^{-t} h^{t^{-1}}\right) \cdot A=\left(\begin{array}{ccc}
0 & 0 & 0 \\
0 & 0 & 0 \\
t & 1 & 0
\end{array}\right)
$$

and thus $p\left(\mathbb{R} \operatorname{Ad}\left(g_{0} g^{-t} h^{t^{-1}}\right) \cdot A\right)$ converges at $t=0$ to $\mathbb{R} \bar{e}_{\alpha}$.

According to the discussion above, this concludes the proof of the lemma.

We are now able to prove the proposition 6.1 .

Proof of the proposition 6.1. Let us assume by contradiction that $\Omega \neq M$. We choose a connected component $O$ of $\tilde{\Omega}$ such that the rank of $\mathrm{D}_{\hat{x}} \mathcal{K}^{\text {tot }}$ for $\hat{x} \in \pi^{-1}(O)$ is maximal among the rank of $\mathrm{D}_{\hat{x}} \mathcal{K}^{\text {tot }}$ for $\hat{x} \in \pi^{-1}(\tilde{\Omega})$. As $\varnothing \neq O \neq \tilde{M}$ by hypothesis, there exists $x \in \partial O$, and as $\tilde{E}^{\alpha} \oplus \tilde{E}^{\beta}$ is contact, [Sus73, Theorem 4.1] implies the existence of a piecewise smooth path $\gamma:[0 ; 1] \rightarrow \tilde{M}$ constituted of a finite concatenation of segments of $\alpha$ and $\beta$-leaves, joining $x=\gamma(1)$ to a point $y=\gamma(0) \in O$. Denoting $t_{0}=\inf \{t \in[0 ; 1] \mid \gamma(t) \in \partial O\}, t_{0}>0$ and $\gamma\left(t_{0}\right) \in \partial O$. Replacing $x$ by $\gamma\left(t_{0}\right)$, keeping only the last smooth arc of $\gamma$, replacing $y$ by the origin of this arc, and choosing a parametrization of this arc by $[0 ; 1]$, we finally end with a smooth path $\gamma:[0 ; 1] \rightarrow \tilde{M}$ such that $\gamma([0 ; 1[) \subset O, x=\gamma(1) \in \partial O$, and $\gamma([0 ; 1])$ is entirely contained in a same $\alpha$ or $\beta$-leaf. The proof being the same in the two cases, we assume that $\gamma([0 ; 1]) \subset \widetilde{\mathcal{F}}^{\alpha}(x)$ to fix the ideas. Denoting $x_{0}=\delta(x), x_{0} \in \mathbf{X} \backslash Y$ according to Lemma 6.2 , and $\delta(\gamma([0 ; 1[)) \subset Y$ because $\delta(O) \subset Y$ (see Lemma 4.2). Finally $\delta\left(\gamma\left([0 ; 1[))\right.\right.$ is an open interval of $C^{\alpha}\left(x_{0}\right)$ contained in $Y$, and $x_{0} \in \mathbf{X} \backslash Y$, i.e. $x_{0} \in \mathcal{G}$. Denoting $\gamma_{0}(t)=\delta(\gamma(t))$, Lemma 6.3 implies therefore that $\mathcal{E}^{c}\left(\gamma_{0}(t)\right)$ converges to a line $D_{0}^{c} \subset\left(\mathcal{E}^{\alpha} \oplus \mathcal{E}^{\beta}\right)\left(x_{0}\right)$ at $t=1$. As $\left.\delta\right|_{O}$ is a local isomorphism between $\left.\tilde{\mathcal{S}}\right|_{O}$ and $\mathcal{S}_{Y}$, we have $\tilde{E}^{c}(\gamma(t))=\left(\mathrm{D}_{\gamma(t)} \delta\right)^{-1}\left(\mathcal{E}^{c}\left(\gamma_{0}(t)\right)\right)$ for any $t \in\left[0 ; 1\left[\right.\right.$, implying $\tilde{E}^{c}(x)=\left(\mathrm{D}_{x} \delta\right)^{-1}\left(D_{0}^{c}\right)$ by continuity. Since $\delta$ is a local isomorphism between the Lagrangian contact structures $\widetilde{\mathcal{L}}$ and $\mathcal{L}_{\mathbf{X}}$, this implies that $\tilde{E}^{c}(x) \subset\left(\tilde{E}^{\alpha} \oplus \tilde{E}^{\beta}\right)(x)$, which contradicts the definition of the transverse distribution $\tilde{E}^{c}$. This contradiction concludes the proof of the proposition.

6.2. Reduction of the holonomy group. We first describe the global and local automorphisms of the models $\left(Y_{\mathbf{t}}, \mathcal{S}_{\mathbf{t}}\right)$ and $\left(Y_{\mathbf{a}}, \mathcal{S}_{\mathbf{a}}\right)$.

Proposition 6.4. (1) $\operatorname{Aut}\left(Y_{\mathbf{t}}, \mathcal{S}_{\mathbf{t}}\right)=H_{\mathbf{t}}=\left[\begin{array}{cc}\mathrm{GL}_{2}(\mathbb{R}) & 0 \\ 0 & 1\end{array}\right]$ and $\operatorname{Aut}\left(Y_{\mathbf{a}}, \mathcal{S}_{\mathbf{a}}\right)=H_{\mathbf{a}}=$ $\mathbf{P}_{\text {min }}$. 
(2) Let $\left(Y, \mathcal{S}_{Y}\right)$ be one of the two models $\left(Y_{\mathbf{t}}, \mathcal{S}_{\mathbf{t}}\right)$ or $\left(Y_{\mathbf{a}}, \mathcal{S}_{\mathbf{a}}\right)$. Then any local isomorphism of $\mathcal{S}_{Y}$ between two connected open subsets of $Y$ is the restriction of the action of a global automorphism of $\operatorname{Aut}\left(Y, \mathcal{S}_{Y}\right)$.

Proof. 1. The inclusions $H_{\mathbf{t}} \subset \operatorname{Aut}\left(Y_{\mathbf{t}}, \mathcal{S}_{\mathbf{t}}\right)$ and $H_{\mathbf{a}} \subset \operatorname{Aut}\left(Y_{\mathbf{a}}, \mathcal{S}_{\mathbf{a}}\right)$ were explained in Paragraphs 4.2.2 and 4.2.3. Since the automorphism groups are contained in the stabilizers of the open subsets, the equalities follow because $H_{\mathbf{t}}=\operatorname{Stab}_{\mathbf{G}}\left(Y_{\mathbf{t}}\right)$ and $H_{\mathbf{a}}=\operatorname{Stab}_{\mathbf{G}}\left(Y_{\mathbf{a}}\right)$. 2. Let us emphasize that $\operatorname{Aut}\left(Y, \mathcal{S}_{Y}\right)$ is precisely the normalizer of $\mathfrak{h}$ in $\mathbf{G}$. Let $\varphi$ be a local automorphism of $\mathcal{S}_{Y}$ between two connected open subsets $U$ and $V$ of $Y$. For any $v \in \mathfrak{h}$, since $\left.v\right|_{V}$ is a Killing field of $\mathcal{S}_{Y}, \varphi^{*}\left(\left.v\right|_{V}\right)$ is a Killing field of $\mathcal{S}_{Y}$, and therefore $\varphi^{*}\left(\left.v\right|_{V}\right)=\left.w\right|_{U}$ for some $w \in \mathfrak{h}$. But $\varphi$ is in particular a local automorphism of the Lagrangian contact structure $\mathcal{L}_{\mathbf{X}}$ of $\mathbf{X}$, and is thus the restriction to an open subset $U \subset Y$ of the left translation by an element $g \in \mathbf{G}$, according to Theorem 2.9. Therefore $\left.w\right|_{U}=\varphi_{*}\left(\left.v\right|_{V}\right)=\left.(\operatorname{Ad}(g) \cdot v)\right|_{U}$, implying that $\operatorname{Ad}(g) \cdot v=w \in \mathfrak{h}$ since the action of $\mathbf{G}$ is analytic (see Lemma 2.16). Consequently, $g \in \operatorname{Nor}_{\mathbf{G}}(\mathfrak{h})=\operatorname{Aut}\left(Y, \mathcal{S}_{Y}\right)$.

Let us recall that $\rho: \pi_{1}(M) \rightarrow \mathbf{G}$ denotes the holonomy morphism associated to the developping map $\delta: \tilde{M} \rightarrow \mathbf{X}$ of the $(\mathbf{G}, \mathbf{X})$-structure of $M$ (see Corollary 3.4 and Paragraph 2.3.2).

Proposition 6.5. The holonomy group $\rho\left(\pi_{1}(M)\right)$ is contained in $\operatorname{Aut}\left(Y, \mathcal{S}_{Y}\right)$. Consequently, $M$ has either a $\left(H_{\mathbf{t}}, Y_{\mathbf{t}}\right)$-structure or a $\left(H_{\mathbf{a}}, Y_{\mathbf{a}}\right)$-structure, and its developping map is a local isomorphism of enhanced Lagrangian contact structures from $\tilde{\mathcal{S}}$ to $\mathcal{S}_{\mathbf{t}}$ (respectively $\left.\mathcal{S}_{\mathbf{a}}\right)$.

Proof. According to Proposition $6.1, \mathcal{S}$ is locally homogeneous, and we thus deduce from Proposition 4.6 that, up to interversion of the distributions $E^{\alpha}$ and $E^{\beta}$, the developping map $\delta$ of the $(\mathbf{G}, \mathbf{X})$-structure of $M$ can be chosen to be a local isomorphism from $(\tilde{M}, \tilde{\mathcal{S}})$ to one of the two models $\left(Y_{\mathbf{t}}, \mathcal{S}_{\mathbf{t}}\right)$ or $\left(Y_{\mathbf{a}}, \mathcal{S}_{\mathbf{a}}\right)$. According to Liouville's theorem 6.4 proved for these two models, the holonomy morphism has moreover values in the corresponding automorphism group $H_{\mathbf{t}}$ (respectively $H_{\mathbf{a}}$ ) described in the same result, and $M$ is finally endowed with a $\left(H_{\mathbf{t}}, Y_{\mathbf{t}}\right)$-structure (resp. $\left(H_{\mathbf{a}}, Y_{\mathbf{a}}\right)$-structure). Concerning the interversion of $E^{\alpha}$ and $E^{\beta}$, it is easy to construct for both models $\left(Y_{\mathbf{t}}, \mathcal{S}_{\mathbf{t}}\right)$ and $\left(Y_{\mathbf{a}}, \mathcal{S}_{\mathbf{a}}\right)$, a diffeomorphism of $Y$ interverting the distributions $\mathcal{E}^{\alpha}$ and $\mathcal{E}^{\beta}$ and fixing the transverse distribution $\mathcal{E}_{Y}^{c}$. In other words for these both models, the structures $\left(\mathcal{E}^{\alpha}, \mathcal{E}^{\beta}, \mathcal{E}_{Y}^{c}\right)$ and $\left(\mathcal{E}^{\beta}, \mathcal{E}^{\alpha}, \mathcal{E}_{Y}^{c}\right)$ are isomorphic, so that a posteriori, the order of the distributions $E^{\alpha}$ and $E^{\beta}$ in the statement of Proposition 6.5 does not matter.

\section{Completeness of the structure}

We will denote by $(\mathcal{H}, Y)$ the local model of $\mathcal{S}$, which is either $\left(H_{\mathbf{t}}, Y_{\mathbf{t}}\right)$ or $\left(H_{\mathbf{a}}, Y_{\mathbf{a}}\right)$, and by $\delta: \tilde{M} \rightarrow Y$ and $\rho: \pi_{1}(M) \rightarrow \mathcal{H}$ the developping map and holonomy morphism of the $(\mathcal{H}, Y)$-structure of $M$. The goal of this section is to prove that:

Proposition 7.1. The developping map $\delta$ is a covering map from $\tilde{M}$ to $Y$.

It is a known fact that a local diffeomorphism satisfying the path-lifting property is a covering map (the reader can for example look for a proof in [DC76, §5.6, Proposition 6 p. 383]). According to the following statement, it will actually be sufficient to prove 
the path-lifting property in the $\alpha, \beta$, and central directions to prove that $\delta$ is a covering map.

Lemma 7.2. Let $h: N \rightarrow B$ be a local diffeomorphism between two smooth threedimensional manifolds, $B$ being connected. We assume that there is a smooth splitting $E_{1} \oplus E_{2} \oplus E_{3}=\mathrm{T} B$ of the tangent bundle of $B$ into three one-dimensional smooth distributions, such that for any $i \in\{1,2,3\}, x \in \operatorname{Im}(h)$, and $\tilde{x} \in h^{-1}(x)$, any path tangent to $E_{i}$ and starting from $x$ entirely lifts through $h$ to a path starting from $\tilde{x}$. Then $h$ is a covering map from $N$ to $B$ (and in particular, $h$ is surjective).

Proof. Since $h$ is a local diffeomorphism, it suffices to prove that our hypothesis implies the lift of any path. By compactness, it is sufficient to locally lift the paths in $B$, around any point. We choose $x \in B$ and a sufficiently small open neighbourhood $U$ of $x$, such that there are three smooth vector fields $X, Y$ and $Z$ generating $E_{1}, E_{2}$ and $E_{3}$ on $U$, and $\varepsilon>0$ such that $(t, u, v) \in]-\varepsilon ; \varepsilon\left[^{3} \mapsto \phi(t, u, v):=\varphi_{X}^{t} \circ \varphi_{Y}^{u} \circ \varphi_{Z}^{v}(x) \in U\right.$ is well-defined, and is a diffeomorphism (this exists according to Inverse mapping theorem). Let us choose $\tilde{x} \in h^{-1}(x)$. Then, denoting by $\tilde{X}=h^{*} X, \tilde{Y}=h^{*} Y$ and $\tilde{Z}=h^{*} Z$ the pullbacks, the property of path-lifting in the directions $E_{1}, E_{2}$ and $E_{3}$, and from any point, implies that $\tilde{\phi}(t, u, v):=\varphi_{\tilde{X}}^{t} \circ \varphi_{\tilde{Y}}^{u} \circ \varphi_{\tilde{Z}}^{v}(\tilde{x})$ is well-defined on $]-\varepsilon ; \varepsilon\left[^{3}\right.$. If $\gamma:[0 ; 1] \rightarrow U$ is a continuous path starting from $x$ and contained in $U$, there are three continuous maps $t, u$ and $v$ from $[0 ; 1]$ to $]-\varepsilon ; \varepsilon[$ such that $\gamma(s)=\phi(t(s), u(s), v(s))$. Since $h \circ \tilde{\phi}=\phi$ by construction, $\tilde{\gamma}(s):=\tilde{\phi}(t(s), u(s), v(s))$ is then a lift of $\gamma$ starting from $\tilde{x}$, which finishes the proof.

Remark 7.3. In our case, proving that the paths in $\delta(\tilde{M})$ in the $\alpha$-direction (respectively $\beta$ or central direction) lift to $\tilde{M}$ is equivalent to prove that for any $x \in \delta(\tilde{M})$ and $\tilde{x} \in \delta^{-1}(x)$, we have the following equality:

$$
\delta\left(\tilde{\mathcal{F}}^{\alpha}(\tilde{x})\right)=\mathcal{C}^{\alpha}(x) \cap \delta(\tilde{M}),
$$

(respectively the same equality for $\beta$-leaves and circes, or for central leaves).

We start by proving that the image of any $\alpha$ (respectively $\beta$ ) leaf in $\tilde{M}$ miss exactly one point in the associated $\alpha$-circle (respectively $\beta$-circle). We recall that $\partial Y=\mathbf{X} \backslash Y$, as explained before Lemma 6.3.

Lemma 7.4. For any $\tilde{x} \in \tilde{M}$, denoting $x=\delta(\tilde{x})$, there exists $x^{*} \in \mathcal{C}^{\beta}(x) \cap \partial Y$ such that $\delta\left(\tilde{\mathcal{F}}^{\beta}(\tilde{x})\right)=\mathcal{C}^{\beta}(x) \backslash\left\{x^{*}\right\}=\mathcal{C}^{\beta}(x) \cap Y$. The same happens for $\alpha$-leaves and their associated $\alpha$-circles.

Proof. We will only write the proof for $\beta$-leaves and $\beta$-circles as in the statement, the case of the $\alpha$-direction being the same. Denoting $\bar{x}=\pi_{M}(\tilde{x})$, and possibly replacing $f$ by $f^{-1}$, we have $\lim _{n \rightarrow+\infty}\left\|\left.\mathrm{D}_{\bar{x}} f^{n}\right|_{E^{\alpha}(\bar{x})}\right\|_{M}=0$ for some fixed Riemannian metric on $M$.

The description of the open subsets $Y_{\mathbf{t}}$ and $Y_{\mathbf{a}}$ in Paragraphs 4.2.2 and 4.2.3 easily shows that in these both cases, the intersection of any $\beta$-circle (respectively $\alpha$-circle) with $Y$ miss exactly one point of the circle. In other words, the intersection $\mathcal{C}^{\beta}(x) \cap \partial Y$ is a single point $\left\{x^{*}\right\}$, and as a consequence $\delta\left(\tilde{\mathcal{F}}^{\beta}(\tilde{x})\right) \subset \mathcal{C}^{\beta}(x) \backslash\left\{x^{*}\right\}=\mathcal{C}^{\beta}(x) \cap Y$. To finish the proof of the lemma, we only have to prove that $\delta\left(\tilde{\mathcal{F}}^{\beta}(\tilde{x})\right)$ cannot miss more 
than one point of $\mathcal{C}^{\beta}(x)$. To achieve this, we assume by contradiction that there exists $x^{-} \neq x^{+} \in \mathcal{C}^{\beta}(x) \backslash\left\{x, x^{*}\right\}$ such that:

$$
\left.\delta\left(\tilde{\mathcal{F}}^{\beta}(\tilde{x})\right)=\right] x^{-} ; x^{+}\left[\subsetneq \mathcal{C}^{\beta}(x) \backslash\left\{x^{*}\right\}\right.
$$

where $] x^{-} ; x^{+}\left[\right.$is the connected component of $\mathcal{C}^{\beta}(x) \backslash\left\{x^{-}, x^{+}\right\}$that contains $x$.

Since $M$ is compact, there exists a strictly increasing sequence $\left(n_{k}\right)$ of positive integers such that, denoting $\bar{x}=\pi_{M}(\tilde{x}), \bar{x}_{k}=f^{n_{k}}(\bar{x})$ converges to a point $\bar{x}_{\infty} \in M$, and as $M=$ $\pi_{1}(M) \backslash \tilde{M}$, there furthermore exists a sequence $\gamma_{k} \in \pi_{1}(M)$ such that $\tilde{x}_{k}:=\gamma_{k} \cdot \tilde{f}^{n_{k}}(\tilde{x})$ converges to a lift $\tilde{x}_{\infty}$ of $\bar{x}_{\infty}$. As $\gamma_{k} \tilde{f}^{n_{k}}$ is an automorphism of the Lagrangian contact structure $\widetilde{\mathcal{L}}$ and $\delta$ a local isomorphism from $\widetilde{\mathcal{L}}$ to $\mathcal{L}_{\mathbf{X}}$, Theorem 2.9 implies the existence of a unique sequence $g_{k} \in \mathbf{G}$ satisfying

$$
\delta\left(\gamma_{k} \cdot \tilde{f}^{n_{k}}(\tilde{x})\right)=g_{k} \cdot \delta(\tilde{x}) .
$$

We denote $x_{k}=\delta\left(\tilde{x}_{k}\right)=g_{k}(x)$, that converges to $x_{\infty}:=\delta\left(\tilde{x}_{\infty}\right)$. Denoting $x_{k}^{-}=g_{k}\left(x^{-}\right)$ and $x_{k}^{+}=g_{k}\left(x^{+}\right), x_{k}, x_{k}^{-}$and $x_{k}^{+}$are three distincts points of $\mathcal{C}^{\beta}\left(x_{k}\right)$ for any $k$. By compactness of $\mathbf{X}$, we can assume up to extraction that $x_{k}^{-}$and $x_{k}^{+}$respectively converge to points $x_{\infty}^{-}$and $x_{\infty}^{+}$of $\mathcal{C}^{\beta}\left(x_{\infty}\right)$, and the hypothesis (7.1) allows us to obtain the following crucial statement.

Fact 7.5. $x_{\infty} \neq x_{\infty}^{-}$, and $x_{\infty} \neq x_{\infty}^{+}$.

Proof. Let us assume by contradiction that $x_{\infty}^{-}=x_{\infty}$. Considering a neighbourhood $U$ of $\tilde{x}_{\infty}$ such that $\left.\delta\right|_{U}$ is injective, we can choose $\tilde{y}_{\infty} \in\left(\tilde{\mathcal{F}}^{\beta}\left(\tilde{x}_{\infty}\right) \cap U\right) \backslash\left\{\tilde{x}_{\infty}\right\}$. There exists a sequence $\tilde{y}_{k} \in \tilde{\mathcal{F}}^{\beta}\left(\tilde{x}_{k}\right)$ converging to $\tilde{y}_{\infty}$, and possibly changing $\tilde{y}_{\infty}$, we can moreover assume that $\left.\delta\left(\tilde{y}_{k}\right) \in\right] x_{k}^{-} ; x_{k}\left[\right.$, implying that $\delta\left(\tilde{y}_{\infty}\right) \in\left[x_{\infty}^{-} ; x_{\infty}\right]$ by continuity. But as $x_{\infty}^{-}=x_{\infty},\left[x_{\infty}^{-} ; x_{\infty}\right]=\left\{x_{\infty}\right\}$, and therefore $\delta\left(\tilde{y}_{\infty}\right)=x_{\infty}=\delta\left(\tilde{x}_{\infty}\right)$, implying $\tilde{y}_{\infty}=\tilde{x}_{\infty}$ by injectivity of $\left.\delta\right|_{U}$, which contradicts our hypothesis on $\tilde{y}_{\infty}$. In the same way, we obtain $x_{\infty} \neq x_{\infty}^{+}$.

The subgroup $\mathrm{SO}(3)$ of $\mathbf{G}$ acts transitively on $\mathbf{X}$, and we can thus choose $\phi \in \mathrm{SO}(3)$ and a sequence $\left(\phi_{k}\right)$ in $\mathrm{SO}(3)$, satisfying $\phi(x)=o$ and $\phi_{k}\left(x_{k}\right)=o$ for any $k$ (we recall that $\left.o=\left(\left[e_{1}\right],\left[e_{1}, e_{2}\right]\right)\right)$. Since $\operatorname{Stab}_{\mathrm{SO}(3)}\left(\mathcal{C}^{\beta}(o)\right)=\left[\begin{array}{cc}\mathrm{SO}(2) & 0 \\ 0 & 1\end{array}\right]$ acts transitively on $\mathcal{C}^{\beta}(o)$, we can moreover assume that $\phi\left(x^{+}\right)=o^{+}$and $\phi_{k}\left(x_{k}^{+}\right)=o^{+}$, where $o^{+}=\left(\left[e_{2}\right],\left[e_{1}, e_{2}\right]\right) \in \mathcal{C}^{\beta}(o)$. For any $k, \phi_{k} \circ g_{k} \circ \phi^{-1}$ is an element of $\operatorname{Stab}_{\mathbf{G}}(o) \cap \operatorname{Stab}_{\mathbf{G}}\left(o^{+}\right)$, i.e. is of the form $\left[\begin{array}{ccc}1 & 0 & x \\ 0 & \lambda_{k} & y \\ 0 & 0 & \mu_{k}\end{array}\right]$. But $\left[\begin{array}{lll}1 & 0 & * \\ 0 & 1 & * \\ 0 & 0 & *\end{array}\right]$ acts trivially in restriction to $\mathcal{C}^{\beta}(o)$, and $A_{k}:=\left[\begin{array}{ccc}1 & 0 & 0 \\ 0 & \lambda_{k} & 0 \\ 0 & 0 & 1\end{array}\right]$ satisfies thus:

$$
\left.g_{k}\right|_{\mathcal{C}^{\beta}(x)}=\left.\phi_{k}^{-1} \circ A_{k} \circ \phi\right|_{\mathcal{C}^{\beta}(x)} .
$$

The following commutative diagram summarizes the situation.

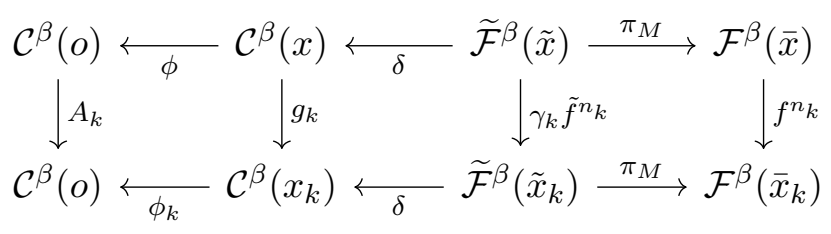


The action of $A_{k} \in \mathbf{G}$ on $\mathcal{C}^{\beta}(o)$ is conjugated to the action of the projective transformations $\left[\begin{array}{cc}1 & 0 \\ 0 & \lambda_{k}\end{array}\right] \in \mathrm{PGL}_{2}(\mathbb{R})$ on $\mathbb{R} \mathbf{P}^{1}$, i.e. to the action of the homotheties of ratio $\lambda_{k}$ on $\mathbb{R} \cup\{\infty\}$. By this conjugation, $o$ corresponds to $0, o^{+}$to $\infty$, and $o^{-}:=$ $\phi\left(x^{-}\right) \in \mathcal{C}^{\beta}(o) \backslash\left\{o, o^{+}\right\}$corresponds to a non-zero point of $\mathbb{R}$. Fact 7.5 implies that $A_{k}\left(o^{-}\right)=\phi_{k}\left(x_{k}^{-}\right) \in \mathcal{C}^{\beta}(o)$ stays bounded away from $o$ (since $\phi_{k} \in \mathrm{SO}(3)$ ), and therefore that $\lambda_{k}$ is bounded away from 0 .

On the other hand, endowing $\tilde{M}$ with the pullback of the Riemannian metric of $M$, the diagramm (7.2) implies $\lim _{k \rightarrow+\infty}\left\|\left.\mathrm{D}_{\tilde{x}}\left(\gamma_{k} \tilde{f}^{n_{k}}\right)\right|_{\tilde{E}^{\beta}(\tilde{x})}\right\|_{\tilde{M}}=0$ (since $\pi_{1}(M)$ acts by isometries). Fixing any Riemannian metric on $\mathbf{X}$, as $\left(\tilde{x}_{k}\right)$ is relatively compact we also have $\lim \left\|\left.\mathrm{D}_{x} g_{k}\right|_{\mathcal{E}^{\beta}(x)}\right\|_{\mathbf{X}}=0$, and since $\left(\phi_{k}\right)$ and $\left(x_{k}\right)$ are relatively compact as well, we finally obtain $\lim \left\|\left.\mathrm{D}_{o} A_{k}\right|_{\mathcal{E}^{\beta}(x)}\right\|_{\mathbf{X}}=0$.

This contradicts the fact that $\lambda_{k}$ is bounded away from 0 , and this contradiction concludes the proof of the lemma.

Lemma 7.4 allows us to easily infer the path-lifting property in the $\alpha$ and $\beta$-directions.

Corollary 7.6. (1) For any $x \in \delta(\tilde{M}), \mathcal{C}^{\alpha}(x) \cap \delta(\tilde{M})=\mathcal{C}^{\alpha}(x) \cap Y$ and $\mathcal{C}^{\beta}(x) \cap$ $\delta(\tilde{M})=\mathcal{C}^{\beta}(x) \cap Y$.

(2) The paths in $\delta(\tilde{M})$ in the $\alpha$ and $\beta$-directions lift to $\tilde{M}$ from any point.

Proof. We only write the proof of the statements for the $\alpha$-direction, the case of the $\beta$-direction being formally the same.

1. For any $\tilde{x} \in \tilde{M}$, denoting $\delta(\tilde{x})=x$, we know that $\partial Y \cap \mathcal{C}^{\alpha}(x)$ is equal to a single point $\left\{x^{*}\right\}$ that satisfies $\mathcal{C}^{\alpha}(x) \backslash\left\{x^{*}\right\}=\mathcal{C}^{\alpha}(x) \cap Y$. Furthermore, $\delta\left(\tilde{\mathcal{F}}^{\alpha}(\tilde{x})\right)=\mathcal{C}^{\alpha}(x) \backslash\left\{x^{*}\right\}=$ $\mathcal{C}^{\alpha}(x) \cap Y$ according to Lemma 7.4. As $\mathcal{C}^{\alpha}(x) \cap \delta(\tilde{M}) \subset \cup_{\tilde{x} \in \delta^{-1}(x)} \delta\left(\tilde{\mathcal{F}}^{\alpha}(\tilde{x})\right)=\mathcal{C}^{\alpha}(x) \cap Y$, we finally obtain $\mathcal{C}^{\alpha}(x) \cap \delta(\tilde{M})=\mathcal{C}^{\alpha}(x) \cap Y$.

2. Together with Lemma 7.4, we finally have $\delta(\tilde{\mathcal{F}}(\tilde{x}))=\mathcal{C}^{\alpha}(x) \cap \delta(\tilde{M})$, for any $x \in \delta(\tilde{M})$ and $\tilde{x} \in \delta^{-1}(x)$. According to the remark 7.3, this proves that any path starting from $x$ in the $\alpha$-direction lifts to $\tilde{M}$ from $\tilde{x}$.

The accessibility property of Lagrangian contact structures allows us to deduce that:

Corollary 7.7. The developping map is surjective: $\delta(\tilde{M})=Y$.

Proof. Let $x$ be a point of the non-empty subset $\delta(\tilde{M})$, and $y$ be any point in $Y$. Restricting the Lagrangian contact structure $\mathcal{L}_{\mathbf{X}}=\left(\mathcal{E}^{\alpha}, \mathcal{E}^{\beta}\right)$ of $\mathbf{X}$ to the connected open subset $Y$, [Sus73, Theorem 4.1] implies the existence of a finite number $x=x_{1}, \ldots, x_{n}=y$ of points of $Y$ such that for any $i=1, \ldots, n-1, x_{i+1} \in \mathcal{C}^{\alpha}\left(x_{i}\right) \cap Y$ or $x_{i+1} \in \mathcal{C}^{\beta}\left(x_{i}\right) \cap Y$. Applying the first statement of Corollary 7.6, we deduce by a direct finite recurrence that for any $i, x_{i} \in \delta(\tilde{M})$, so that $y \in \delta(\tilde{M})$.

We finally prove that the central paths also lift, by a specific method for each model.

Lemma 7.8. In the case of $Y_{\mathbf{t}}$, any central path starting at any point $x \in Y_{\mathbf{t}}$ lifts in $\tilde{M}$ from any point $\tilde{x} \in \delta^{-1}(x)$. 
Proof. Let us recall that $H_{\mathbf{t}}=\left[\begin{array}{cc}\mathrm{GL}_{2}(\mathbb{R}) & 0 \\ 0 & 1\end{array}\right]=\operatorname{Aut}\left(Y_{\mathbf{t}}, \mathcal{S}_{\mathbf{t}}\right)$ and $o_{\mathbf{t}}=\left([1,0,1],\left[(1,0,1), e_{2}\right]\right) \in$ $Y_{\mathbf{t}}$. Since $Z=\left(\begin{array}{ccc}1 & 0 & 0 \\ 0 & 1 & 0 \\ 0 & 0 & -2\end{array}\right)$ is central in $\mathfrak{h}_{\mathbf{t}}$, the Killing field $Z^{\dagger}$ of $\mathcal{S}_{\mathbf{t}}$ associated to $Z$ is $H_{\mathbf{t}^{-}}$ invariant. As $\mathcal{E}_{\mathbf{t}}^{c}\left(o_{\mathbf{t}}\right)=\mathbb{R} Z^{\dagger}\left(o_{\mathbf{t}}\right)$ (see Paragraph 4.2.2) and $\mathcal{E}^{c}$ is $H_{\mathbf{t}}$-invariant as well, $Z^{\dagger}$ actually generates the transverse distribution on $Y_{\mathbf{t}}$. At any point $x \in Y_{\mathbf{t}}$, we thus have $\mathcal{F}_{\mathbf{t}}^{c}(x)=\exp (\mathbb{R} Z) \cdot x$. Now, as the holonomy group $\rho\left(\pi_{1}(M)\right)$ is contained in $H_{\mathbf{t}}$ according to Proposition 6.5 , it leaves $Z^{\dagger}$ invariant, and the pullback $\tilde{X}:=\delta^{*} Z^{\dagger}$ is thus preserved by the fundamental group $\pi_{1}(M)$. This allows us to push $\tilde{X}$ down on $M$, to a Killing field $X$ generating the central direction $E^{c}$. As $M$ is compact, $X$ is a complete vector field, and as $\pi_{M}: \tilde{M} \rightarrow M$ is a covering map, the pullback $\pi_{M}^{*} X=\tilde{X}$ is also complete, implying that for any $\tilde{x} \in \tilde{M}$, the central leaf at $\tilde{x}$ is simply the integral curve of $\tilde{X}=\delta^{*} Z^{\dagger}$ at $\tilde{x}$. For any $x \in Y_{\mathbf{t}}$ and $\tilde{x} \in \delta^{-1}(x)$ (which is non-empty because $\delta(\tilde{M})=Y_{\mathbf{t}}$ according to Corollary 7.7) we thus have $\delta\left(\tilde{\mathcal{F}}^{c}(\tilde{x})\right)=\left\{\delta\left(\varphi_{\tilde{X}}^{t}(\tilde{x})\right) \mid t \in \mathbb{R}\right\}=\exp (\mathbb{R} Z) \cdot x=\mathcal{F}_{\mathbf{t}}^{c}(x)$. This finishes the proof of the lemma according to Remark 7.3.

Lemma 7.9. In the case of $Y_{\mathbf{a}}$, any central path starting at any point $x \in Y_{\mathbf{a}}$ lifts in $\tilde{M}$ from any point $\tilde{x} \in \delta^{-1}(x)$.

Proof. Let us first emphasize that the argument used in the previous lemma for the case of $Y_{\mathbf{t}}$ does not work here, because the center of $\mathfrak{h}_{\mathbf{a}}$ is trivial.

We identify $Y_{\mathbf{a}}$ with $\mathbb{R}^{3}$ through $(x, y, z) \in \mathbb{R}^{3} \mapsto([x, y, 1],[(x, y, 1),(z, 1,0)]) \in Y_{\mathbf{a}}$, and we consider the following vector fields of $Y_{\mathbf{a}}$ in these global coordinates:

$$
X^{\alpha}(x, y, z)=e_{3}, X^{\beta}(x, y, z)=(z, 1,0), X^{c}(x, y, z)=e_{1} .
$$

These vector fields are complete and generate the enhanced Lagrangian contact structure $\mathcal{S}_{\mathbf{a}}=\left(\mathcal{E}^{\alpha}, \mathcal{E}^{\beta}, \mathcal{E}_{\mathbf{a}}^{c}\right)$ on $Y_{\mathbf{a}}$ (see Paragraph 4.2.3). Since the paths tangent to the $\alpha$ and $\beta$ distributions entirely lift to $\tilde{M}$ according to Corollary 7.6, we deduce that the pullbacks $\tilde{X}^{\alpha}=\delta^{*} X^{\alpha}$ and $\tilde{X}^{\beta}=\delta^{*} X^{\beta}$ are complete as well. We can furthermore realize the flow of the central vector field $X^{c}$ by $\alpha-\beta$ curves through the following equalities:

$$
\left\{\begin{array}{l}
\varphi_{X^{\beta}}^{-t} \circ \varphi_{X^{\alpha}}^{-t} \circ \varphi_{X^{\beta}}^{t} \circ \varphi_{X^{\alpha}}^{t}(x)=x+t^{2} e_{1}=\varphi_{X^{c}}^{t^{2}}(x), \\
\varphi_{X^{\beta}}^{t} \circ \varphi_{X^{\alpha}}^{-t} \circ \varphi_{X^{\beta}}^{-t} \circ \varphi_{X^{\alpha}}^{t}(x)=x-t^{2} e_{1}=\varphi_{X^{c}}^{t^{2}}(x) .
\end{array}\right.
$$

The same equalities are thus true for the pullbacks $\tilde{X}^{\alpha}, \tilde{X}^{\beta}$, and $\tilde{X}^{c}=\delta^{*} X^{c}$, and since the flows of $\tilde{X}^{\alpha}$ and $\tilde{X}^{\beta}$ are defined for all times, these equalities show that $\tilde{X}^{c}$ is also complete. The completeness of $\tilde{X}^{c}$ allows us to lift any central path of $Y_{\mathbf{a}}$ from any point of $\tilde{M}$, and concludes the proof of the lemma.

End of the proof of Proposition 7.1. According to Corollary 7.6 and to Lemmas 7.8 and 7.9, the local diffeomorphism $\delta$ satisfies the path-lifting property on $Y$ in the $\alpha, \beta$, and central directions, and is thus a covering map from $\tilde{M}$ to $Y$ according to Lemma 7.2.

\section{Conclusion}

8.1. End of the proof of Theorem B. The work that has been done so far tells us that for one of the two models $\left(\mathcal{H}, Y, \mathcal{S}_{Y}\right)=\left(H_{\mathbf{t}}, Y_{\mathbf{t}}, \mathcal{S}_{\mathbf{t}}\right)$ or $\left(H_{\mathbf{a}}, Y_{\mathbf{a}}, \mathcal{S}_{\mathbf{a}}\right), M$ is a $(\mathcal{H}, Y)$ manifold whose developping map $\delta: \tilde{M} \rightarrow Y$ is a covering map satisfying $\delta^{*} \mathcal{S}_{Y}=\tilde{\mathcal{S}}$. With these informations, we will finish the proof of Theorem B. We will use the link 
between the geometrical and algebraic point of views on the models $\left(Y_{\mathbf{t}}, \mathcal{S}_{\mathbf{t}}\right)$ and $\left(Y_{\mathbf{a}}, \mathcal{S}_{\mathbf{a}}\right)$, explained in Paragraphs 4.1 and 4.2 .

8.1.1. Case of $\left(Y_{\mathbf{a}}, \mathcal{S}_{\mathbf{a}}\right)$. We first assume that $(M, \mathcal{S})$ is locally isomorphic to $\left(Y_{\mathbf{a}}, \mathcal{S}_{\mathbf{a}}\right)$. Since $Y_{\mathbf{a}}$ is simply connected (because homeomorphic to Heis(3)), the covering map $\delta: \tilde{M} \rightarrow Y_{\text {a }}$ is actually a diffeomorphism in this case. Since the developping map conjugates the action of $\pi_{1}(M)$ on $\tilde{M}$ to the action of the holonomy group $\Gamma=\rho\left(\pi_{1}(M)\right) \subset H_{\mathbf{a}}$ on $Y_{\mathbf{a}}$, we can assume without lost of generality that $M$ is a compact quotient $\Gamma \backslash Y_{\mathbf{a}}$, with $\Gamma$ a discrete subgroup of $H_{\mathbf{a}}$ acting freely, properly, and cocompactly. Since $f$ is an automorphism of $(M, \mathcal{S})$, we moreover deduce from Proposition 6.4 that $f \in \operatorname{Nor}_{H_{\mathrm{a}}}(\Gamma)$.

We saw in Paragraph 4.2.3 that the identification between Heis(3) and $Y_{\mathbf{a}}$ given by the orbital map at $o_{\mathbf{a}}$ conjugates the action of $H_{\mathbf{a}}$ on $Y_{\mathbf{a}}$, and the action of the semi-direct product Heis(3) $\rtimes \mathcal{A}$ of affine automorphisms of Heis(3) preserving its left-invariant structure. We can thus assume that $M$ is a quotient $\Gamma \backslash \operatorname{Heis}(3)$, with $\Gamma$ a discrete subgroup of Heis(3) $\rtimes \mathcal{A}$ acting freely, properly, and cocompactly on Heis(3), and that $f \in \operatorname{Nor}_{\mathrm{Heis}(3) \rtimes \mathcal{A}}(\Gamma)$.

Denoting $[x, y, z]=\left(\begin{array}{lll}1 & x & z \\ 0 & 1 & y \\ 0 & 0 & 1\end{array}\right)$, the identification $[x, y, z] \in \operatorname{Heis}(3) \mapsto(x, y, z) \in \mathbb{R}^{3}$ of Heis(3) with $\mathbb{R}^{3}$ is equivariant for the following injective morphism from Heis(3) $\rtimes \mathcal{A}$ to the affine transformations of $\mathbb{R}^{3}$ :

$$
\Theta:\left([x, y, z], \varphi_{\lambda, \mu}\right) \in \operatorname{Heis}(3) \rtimes \mathcal{A} \mapsto\left(\begin{array}{ccc}
\lambda & 0 & 0 \\
0 & \mu & 0 \\
0 & \mu x & \lambda \mu
\end{array}\right)+\left[\begin{array}{c}
x \\
y \\
z
\end{array}\right] \in \operatorname{Aff}\left(\mathbb{R}^{3}\right) .
$$

$M$ is thus diffeomorphic to the quotient $\Lambda \backslash \mathbb{R}^{3}$, where $\Lambda:=\Theta(\Gamma)$ is a discrete subgroup of affine transformations of $\mathbb{R}^{3}$ contained in $S:=\Theta(\operatorname{Heis}(3) \rtimes \mathcal{A})$, acting freely, properly and cocompactly on $\mathbb{R}^{3}$. Since $S$ is solvable (because Heis(3) $\rtimes \mathcal{A} \simeq \mathbf{P}_{\min }$ is), the work of Fried and Goldmann in [FG83] (more precisely Theorem 1.4, Corollary 1.5 and Paragraphs 3 and 4 of this paper) implies the existence of a so-called crystallographic hull $C$ of $\Lambda$. This group $C$ is a closed subgroup of $S$ containing $\Lambda$, and whose identity component $C^{0}$ satisfies the following assumptions: $\Lambda \cap C^{0}$ has finite index in $\Lambda$ and is cocompact in $C^{0}, C^{0}$ acts simply transitively on $\mathbb{R}^{3}$, and $C^{0}$ is isomorphic to $\mathbb{R}^{3}$, Heis $(3)$, or Sol. One can easily check that $S$ does not contain any subgroup isomorphic to $\mathbb{R}^{3}$, that the subgroups of $S$ isomorphic to $S o l$ do not act simply transitively on $\mathbb{R}^{3}$, and that $\Theta(\operatorname{Heis}(3))$ is the only subgroup of $S$ isomorphic to Heis(3). Finally, $C^{0}$ is equal to $\Theta(\operatorname{Heis}(3))$, and therefore, $\Lambda \cap \Theta(\operatorname{Heis}(3))$ has finite index in $\Lambda$ and is cocompact in $\Theta(\operatorname{Heis}(3))$. As a consequence, $\Gamma_{0}:=\Gamma \cap \operatorname{Heis}(3)$ has finite index in $\Gamma$ and is a cocompact lattice of Heis(3).

The morphism $p:(g, \varphi) \in \operatorname{Heis}(3) \rtimes \mathcal{A} \mapsto \varphi \in \mathcal{A}$ having a kernel equal to Heis(3), $\Gamma / \Gamma_{0}$ is isomorphic to $p(\Gamma) \subset \mathcal{A}$. But $\mathcal{A}$ is isomorphic to $\left(\mathbb{R}^{*}\right)^{2}$, and a finite subgroup of $\mathcal{A}$ is thus contained in the subgroup $\left\{\varphi_{ \pm 1, \pm 1}\right\}$ of cardinal 4 , implying that $\Gamma_{0}$ is a subgroup of $\Gamma$ of index at most 4. Let us denote $f=(g, \varphi) \in \operatorname{Nor}_{H e i s(3) \rtimes \mathcal{A}}(\Gamma)$. Then we have $g \varphi\left(\Gamma_{0}\right) g^{-1}=\Gamma_{0}$, and the affine automorphism $x \mapsto g \varphi(x)$ induces therefore a diffeomorphism $\check{f}$ of $\check{M}:=\Gamma_{0} \backslash \operatorname{Heis}(3)$ through $\check{f}\left(x \Gamma_{0}\right)=g \varphi(x) \Gamma_{0}$. The canonical 
projection $\check{\pi}: \check{M}=\Gamma_{0} \backslash \operatorname{Heis}(3) \rightarrow M=\Gamma \backslash \operatorname{Heis}(3)$ is a covering of finite order equal to the index of $\Gamma_{0}$ in $\Gamma$, and we have $\check{\pi} \circ \check{f}=f \circ \check{\pi}$.

We know that $\varphi$ is equal to $\varphi_{\lambda, \mu}$ for some $(\lambda, \mu) \in\left(\mathbb{R}^{*}\right)^{2}$ (see (1.1)), and it only remains to show that $|\lambda|<1$ and $|\mu|>1$, or the contrary, to conclude that $\check{f}$ is a partially hyperbolic affine automorphism of Heis(3). Let us assume by contradiction that $|\lambda|<1$ and $|\mu|<1$. Choosing a left-invariant volume form $\nu$ on Heis(3), we have $\left(\left(\mathrm{D}_{e} \varphi\right)^{*} \nu\right)_{e}=\lambda^{2} \mu^{2} \nu_{e}$, and $\nu$ induces a volume form $\bar{\nu}$ on $\check{M}=\Gamma_{0} \backslash \operatorname{Heis}(3)$ such that $\check{f}^{*} \bar{\nu}=\lambda^{2} \mu^{2} \bar{\nu}$, because $L_{g}$ preserves $\nu$. As $\check{f}$ is a diffeomorphism of the compact manifold $\check{M}$, we must have $\int_{\check{M}} \bar{\nu}=\int_{\check{M}} \check{f}^{*} \bar{\nu}=\lambda^{2} \mu^{2} \int_{\check{M}} \bar{\nu}$, which is a contradiction because $\int_{\check{M}} \bar{\nu} \neq 0$ and $\lambda^{2} \mu^{2}<1$. The same argument shows that we cannot have $|\lambda|>1$ and $|\mu|>1$ neither, which finishes the proof of Theorem B in the case of the local model $\left(Y_{\mathbf{a}}, \mathcal{S}_{\mathbf{a}}\right)$.

8.1.2. Case of $\left(Y_{\mathbf{t}}, \mathcal{S}_{\mathbf{t}}\right)$. We now assume that $\mathcal{S}$ is locally isomorphic to $\left(Y_{\mathbf{t}}, \mathcal{S}_{\mathbf{t}}\right)$. Identifying $Y_{\mathbf{t}}$ with $\mathrm{SL}_{2}(\mathbb{R})$ as explained in Paragraph 4.2.2, we can lift the developping map $\delta: \tilde{M} \rightarrow Y_{\mathbf{t}}$ to a map $\tilde{\delta}: \tilde{M} \rightarrow \widetilde{\mathrm{SL}}_{2}(\mathbb{R})$ through the universal cover morphism $\pi_{\mathrm{SL}_{2}(\mathbb{R})}: \widetilde{\mathrm{SL}}_{2}(\mathbb{R}) \rightarrow \mathrm{SL}_{2}(\mathbb{R})$. As $\delta$ is a covering map according to Proposition 7.1, $\tilde{\delta}$ is a diffeomorphism because $\widetilde{\mathrm{SL}}_{2}(\mathbb{R})$ is simply connected. As $M$ is supposed to be orientable, $\pi_{1}(M)$ preserves its orientation, implying that the holonomy group $\rho\left(\pi_{1}(M)\right)$ is contained in the subgroup $H_{\mathrm{t}}^{+}=\mathrm{GL}_{2}^{+}(\mathbb{R})$ of elements of positive determinant. We saw in Paragraph 4.2.2 that the diffeomorphism $\theta_{O_{\mathbf{t}}} \circ \iota: \mathrm{SL}_{2}(\mathbb{R}) \rightarrow Y_{\mathbf{t}}$ conjugates the action of $\mathrm{GL}_{2}^{+}(\mathbb{R})$ on $Y_{\mathbf{t}}$ and the action of $\mathrm{SL}_{2}(\mathbb{R}) \times A$ on $\mathrm{SL}_{2}(\mathbb{R})$. As $\pi_{\mathrm{SL}_{2}(\mathbb{R})}$ is equivariant for the projection $\widetilde{\mathrm{SL}}_{2}(\mathbb{R}) \times \tilde{A} \rightarrow \mathrm{SL}_{2}(\mathbb{R}) \times A$, we finally conclude that the diffeomorphism $\tilde{\delta}: \tilde{M} \rightarrow \widetilde{\mathrm{SL}}_{2}(\mathbb{R})$ is equivariant for a morphism $\tilde{\rho}: \pi_{1}(M) \rightarrow \widetilde{\mathrm{SL}}_{2}(\mathbb{R}) \times \tilde{A}$. We can thus assume that $M$ is a quotient $\tilde{\Gamma} \backslash \widetilde{S L}_{2}(\mathbb{R})$, with $\tilde{\Gamma}$ a discrete subgroup of $\widetilde{S L}_{2}(\mathbb{R}) \times \tilde{A}$ acting freely, properly, and cocompactly on $\widetilde{\mathrm{SL}}_{2}(\mathbb{R})$. Possibly replacing $f$ by $f^{2}$, we can assume that $f$ preserves the orientation of $M$, and Proposition 6.4 implies then that $f=L_{g} \circ R_{a^{t}}$ with $\left(g, a^{t}\right) \in \operatorname{Nor}_{\widetilde{S L}_{2}(\mathbb{R}) \times \tilde{A}}(\tilde{\Gamma})$.

Denoting by $r_{1}: \widetilde{\mathrm{SL}}_{2}(\mathbb{R}) \times \tilde{A} \rightarrow \widetilde{\mathrm{SL}}_{2}(\mathbb{R})$ the projection on the first factor, and $\tilde{\Gamma}_{0}:=$ $r_{1}(\tilde{\Gamma}) \subset \widetilde{\mathrm{SL}}_{2}(\mathbb{R})$, we now prove the following result.

Fact 8.1. $\tilde{\Gamma}_{0}$ is a cocompact lattice of $\widetilde{\mathrm{SL}}_{2}(\mathbb{R})$, and $\tilde{\Gamma}$ is the graph-group $\operatorname{gr}\left(\tilde{u}, \tilde{\Gamma}_{0}\right)$ of a morphism $\tilde{u}: \tilde{\Gamma} \rightarrow \tilde{A}$.

Proof. Choosing a generator $z$ of the center $\tilde{\mathcal{Z}}$ of $\widetilde{S L}_{2}(\mathbb{R})$, the finiteness of the level proved by Salein in [Sal99, Theorem 3.3.2.3] implies the existence of a non-zero integer $k \in \mathbb{N}^{*}$ such that $\tilde{\Gamma} \cap(\tilde{\mathcal{Z}} \times\{e\})=\left\langle\left(z^{k}, e\right)\right\rangle$. We will denote by $\langle g\rangle$ the group generated by an element $g$, and we introduce the group $\operatorname{PSL}_{2}^{(k)}(\mathbb{R}):=\widetilde{\mathrm{SL}}_{2}(\mathbb{R}) /\left\langle z^{k}\right\rangle$ and denote by $p_{k}: \widetilde{\mathrm{SL}}_{2}(\mathbb{R}) \rightarrow \mathrm{PSL}_{2}^{(k)}(\mathbb{R})$ its universal cover. Then, denoting $A_{k}=p_{k}(\tilde{A})$ and $\Gamma_{k}:=\left(p_{k} \times\right.$ $\left.p_{k}\right)(\tilde{\Gamma})<\operatorname{PSL}_{2}^{(k)}(\mathbb{R}) \times A_{k}, p_{k}$ induces a diffeomorphism $\bar{p}_{k}: \tilde{\Gamma} \backslash \widetilde{\mathrm{SL}}_{2}(\mathbb{R}) \rightarrow \Gamma_{k} \backslash \mathrm{PSL}_{2}^{(k)}(\mathbb{R})$ (because $\operatorname{Ker} p_{k}=\left\langle z^{k}\right\rangle$ and $\left(z^{k}, e\right) \in \tilde{\Gamma}$ ), implying in particular that $\Gamma_{k}$ acts freely, properly, and cocompactly on $\operatorname{PSL}_{2}^{(k)}(\mathbb{R})$. 
We can now apply the work of Kulkarni-Raymond in [KR85] to $\Gamma_{k}$. We denote by $\pi: \widetilde{\mathrm{SL}}_{2}(\mathbb{R}) \rightarrow \mathrm{PSL}_{2}(\mathbb{R})$ the universal cover morphism of $\mathrm{PSL}_{2}(\mathbb{R})$ (of kernel $\tilde{\mathcal{Z}}$ ), and by $\pi_{k}: \operatorname{PSL}_{2}^{(k)}(\mathbb{R}) \rightarrow \mathrm{PSL}_{2}(\mathbb{R})$ the induced $k$-fold covering by $\mathrm{PSL}_{2}^{(k)}(\mathbb{R})$. Then, with $\Gamma=(\pi \times \pi)(\tilde{\Gamma})$ and $\Gamma_{0}=r_{1}(\Gamma)<\mathrm{PSL}_{2}(\mathbb{R})$ the projection on the first factor, the form [Tho14, Lemma 4.3.1] of Kulkarni-Raymond's results proved by Tholozan implies that $\Gamma_{0}$ is a cocompact lattice of $\mathrm{PSL}_{2}(\mathbb{R})$, and that $\left.\pi_{k} \circ r_{1}\right|_{\Gamma_{k}}$ is injective.

The first assertion ensures that $\tilde{\Gamma}_{0}$ is discrete in $\widetilde{\mathrm{SL}}_{2}(\mathbb{R})$. The second one implies that $\Gamma=\operatorname{gr}\left(u, \Gamma_{0}\right)$ is the graph-group of a morphism $u: \Gamma_{0} \rightarrow A=\pi(\tilde{A})$. Since $\left.r_{1}\right|_{\tilde{\Gamma}}$ is also injective, this implies that $\tilde{\Gamma}$ is the graph of a morphism $\tilde{u}: \tilde{\Gamma}_{0} \rightarrow \tilde{A}$, trivial on $\tilde{\Gamma}_{0} \cap \tilde{\mathcal{Z}}$.

Since $\tilde{\mathcal{Z}} \cap \tilde{\Gamma}_{0}=\left\langle z^{k}\right\rangle$ is finite, the projection $\tilde{\Gamma}_{0} \backslash \widetilde{S L}_{2}(\mathbb{R}) \rightarrow \Gamma_{0} \backslash \mathrm{PSL}_{2}(\mathbb{R})$ has finite fibers, implying that $\tilde{\Gamma}$ is a cocompact lattice as $\Gamma_{0} \backslash \mathrm{PSL}_{2}(\mathbb{R})$ is compact.

The projection $\Gamma_{0}=\pi\left(\tilde{\Gamma}_{0}\right)$ is a cocompact lattice of $\mathrm{PSL}_{2}(\mathbb{R})$ according to the proof of Fact 8.1, and $\Gamma_{0} \backslash \operatorname{Nor}_{\mathrm{PSL}_{2}(\mathbb{R})}\left(\Gamma_{0}\right)$ is thus finite. Therefore, $\tilde{\Gamma}_{0} \backslash \operatorname{Nor}_{\widetilde{S L}_{2}(\mathbb{R})}\left(\tilde{\Gamma}_{0}\right)$ is finite as well since the projection $\tilde{\Gamma}_{0} \backslash \operatorname{Nor}_{\widetilde{S L}_{2}(\mathbb{R})}\left(\tilde{\Gamma}_{0}\right) \rightarrow \Gamma_{0} \backslash \operatorname{Nor}_{\mathrm{PSL}_{2}(\mathbb{R})}\left(\Gamma_{0}\right)$ has finite fibers $\left(\tilde{\mathcal{Z}} \cap \tilde{\Gamma}_{0}=\left\langle z^{k}\right\rangle\right.$ is finite according to the finiteness of the level).

Recall that $f=L_{g} \circ R_{a}$, where $\left(g, a^{t}\right) \in \operatorname{Nor}_{\widetilde{S L}_{2}(\mathbb{R}) \times \tilde{A}}(\tilde{\Gamma})$. Therefore $g \in \operatorname{Nor}_{\widetilde{S L}_{2}(\mathbb{R})}\left(\tilde{\Gamma}_{0}\right)$, and since $\tilde{\Gamma}_{0} \backslash \operatorname{Nor}_{\widetilde{S L}_{2}(\mathbb{R})}\left(\tilde{\Gamma}_{0}\right)$ is finite, there exists $n \in \mathbb{N}^{*}$ such that $\gamma:=g^{n} \in \tilde{\Gamma}_{0}$. Denoting $a:=a^{n} \tilde{u}(\gamma)^{-1}$, we have $f^{n}=L_{\gamma} \circ R_{a^{n}}=R_{a} \circ\left(L_{\gamma} \circ R_{\tilde{u}(\gamma)}\right)$. But $L_{\gamma} \circ R_{\tilde{u}(\gamma)}$ acts trivially on the quotient $\tilde{\Gamma} \backslash \widetilde{\mathrm{SL}}_{2}(\mathbb{R})$, and therefore $f=R_{a}$ is a non-zero time-map of the algebraic contact-Anosov flow $\left(R_{a^{t}}\right)$ on $\tilde{\Gamma} \backslash \widetilde{\mathrm{SL}}_{2}(\mathbb{R})$.

Let us underline that $\left(R_{a^{t}}\right)$ is indeed Anosov, because the work of Zeghib in [Zeg96, Prop. 4.2 p.868] proves that $\left(R_{a^{t}}\right)$ is quasi-Anosov with the definition of Mañé, and Mañé proves in [Mañ77, Theorem A] that three-dimensional quasi-Anosov flows are Anosov.

This concludes the proof of Theorem B in the case where $\mathcal{S}$ is locally isomorphic to $\left(Y_{\mathbf{t}}, \mathcal{S}_{\mathbf{t}}\right)$, and concludes thus its whole proof.

8.2. Proof of Theorem A. Theorem B directly implies Theorem A stated in the introduction thanks to an argument of Brin. More precisely, we obtain the following refined version of Theorem $\mathrm{A}$, where no domination is required on the central direction, and where the two remaining directions can a priori be both contracted, or both expanded.

Corollary 8.2. Let $M$ be a closed, connected and orientable three-dimensional manifold, endowed with a smooth splitting $\mathrm{T} M=E^{\alpha} \oplus E^{\beta} \oplus E^{c}$ such that $E^{\alpha} \oplus E^{\beta}$ is a contact distribution. Let $f$ be a diffeomorphism of $M$ that preserves this splitting, and such that

- each of the distributions $E^{\alpha}$ and $E^{\beta}$ is either uniformly contracted, or uniformly expanded by $f$,

- and $N W(f)=M$.

Then the conclusions of Theorem A hold. In particular, $f$ is a partially hyperbolic diffeomorphism.

Proof. Since $E^{\alpha} \oplus E^{\beta}$ is contact and $M$ connected, any two points of $M$ are linked by the concatenation of a finite number of paths, tangent either to $E^{\alpha}$ or to $E^{\beta}$ (this is for example a consequence of the work of Sussmann in [Sus73, Theorem 4.1]). In other 
words, the pair $\left(\mathcal{F}^{\alpha}, \mathcal{F}^{\beta}\right)$ of foliations associated to $\left(E^{\alpha}, E^{\beta}\right)$ is topologically transitive in the terminology of Brin in [Bri75]. Our hypothesis of uniform contraction or expansion of the distributions $E^{\alpha}$ and $E^{\beta}$ directly implies that $\mathcal{F}^{\alpha}$ and $\mathcal{F}^{\beta}$ are uniformly contracted or expanded in the terminology of [Bri75]. Since $N W(f)=M$ by hypothesis, [Bri75, Theorem 1.1] implies that $f$ is topologically transitive. In fact, although Brin states his result assuming that one of the distributions is contracted, and the other one expanded, it is easy to see that his proof does in fact not use this assumption, and that the same proof works if both distributions are expanded, or both contracted.

We are now under the hypotheses of Theorem B, and its conclusions hold.

\section{REFERENCES}

[Bar10] Thierry Barbot. Three-dimensional Anosov flag manifolds. Geometry \& Topology, 14(1):153191, 2010.

[BFL92] Yves Benoist, Patrick Foulon, and François Labourie. Flots d'Anosov à distributions stable et instable différentiables. Journal of the American Mathematical Society, 5(1):33-74, 1992.

[BFM09] Uri Bader, Charles Frances, and Karin Melnick. An Embedding Theorem for Automorphism Groups of Cartan Geometries. Geometric and Functional Analysis, 19(2):333-355, September 2009.

[Bri75] M. I. Brin. Topological transitivity of one class of dynamic systems and flows of frames on manifolds of negative curvature. Functional Analysis and Its Applications, 9(1):8-16, January 1975.

[BZ19] Christian Bonatti and Jinhua Zhang. Transitive partially hyperbolic diffeomorphisms with one-dimensional neutral center. arXiv:1904.05295 [math], April 2019.

[CP15] Sylvain Crovisier and Rafael Potrie. Introduction to partially hyperbolic dynamics, Lecture notes for a minicourse at ICTP, July 2015. Available on the web-pages of the authors

[CPRH19] Pablo D. Carrasco, Enrique Pujals, and Federico Rodriguez-Hertz. Classification of partially hyperbolic diffeomorphisms under some rigid conditions. arXiv:1903.09264 [math], March 2019.

[ČS09] Andreas Čap and Jan Slovák. Parabolic geometries I Background and general theory, volume 154 of Mathematical Surveys and Monographs. American Mathematical Society, Providence, RI, 2009.

[DC76] Manfredo Do Carmo. Differential Geometry of Curves and Surfaces. Prentice Hall, 1976.

[DK16] Boris Doubrov and Boris Komrakov. The geometry of second-order ordinary differential equations. arXiv:1602.00913 [math], February 2016.

[FG83] David Fried and William M Goldman. Three-dimensional affine crystallographic groups. $A d-$ vances in Mathematics, 47(1):1-49, January 1983.

[Fra16] Charles Frances. Variations on Gromov's open-dense orbit theorem. Bulletin de la Société mathématique de France, 146, May 2016.

[GD91] Mikhail Gromov and Giuseppina D'Ambra. Lectures on transformation groups : geometry and dynamics. Surveys in differential geometry, 1991.

[Ghy87] Étienne Ghys. Flots d'Anosov dont les feuilletages stables sont différentiables. Annales Scientifiques de l'École Normale Supérieure. Quatrième Série, 20(2):251-270, 1987.

[Gro88] Michael Gromov. Rigid transformations groups. Géométrie différentielle (Paris, 1986), 33:65139, 1988.

[HP18] Andy Hammerlindl and Rafael Potrie. Partial hyperbolicity and classification: a survey. Ergodic Theory and Dynamical Systems, 38(2), April 2018.

[Kan88] Masahiko Kanai. Geodesic flows of negatively curved manifolds with smooth stable and unstable foliations. Ergodic Theory and Dynamical Systems, 8(2):215-239, June 1988.

[Kob95] Shoshichi Kobayashi. Transformation Groups in Differential Geometry. Classics in Mathematics. Springer-Verlag, Berlin Heidelberg, 1995. 
[KR85] Ravi S. Kulkarni and Frank Raymond. 3-dimensional Lorentz space-forms and Seifert fiber spaces. Journal of Differential Geometry, 21(2):231-268, 1985.

[KT17] Boris Kruglikov and Dennis The. The gap phenomenon in parabolic geometries. Journal für die reine und angewandte Mathematik (Crelles Journal), 2017(723), January 2017.

[Mañ77] Ricardo Mañé. Quasi-Anosov Diffeomorphisms and Hyperbolic Manifolds. Transactions of the American Mathematical Society, 229:351-370, 1977.

[Pal57] Richard Palais. A global formulation of the Lie theory of transformation groups. Memoirs of the American Mathematical Society, January 1957.

[Pec16] Vincent Pecastaing. On two theorems about local automorphisms of geometric structures. Annales de l'institut Fourier, 66(1):175-208, 2016.

[Sal99] François Salein. Variétés anti-de-Sitter de dimension 3. Thèse de doctorat, École normale supérieure de Lyon, 1999.

[Sam89] Pierre Samuel. Géométrie projective. Presses universitaires de France. 1989.

[Sha97] R.W. Sharpe. Differential geometry: Cartan's generalization of Klein's Erlangen program. Foreword by S. S. Chern. Berlin: Springer, 1997.

[Sus73] Héctor J. Sussmann. Orbits of families of vector fields and integrability of distributions. Transactions of the American Mathematical Society, 180:171-188, 1973.

[Tho14] Nicolas Tholozan. Uniformisation des variétés pseudo-riemanniennes localement homogènes. Thèse de doctorat, Université Nice Sophia Antipolis, 2014.

[Thu97] William P. Thurston. Three-Dimensional Geometry and Topology, Volume 1: Volume 1. Princeton University Press, 1997.

[Tre96] A. Tresse. Détermination des invariants ponctuels de l'équation différentielle ordinaire $d u$ second ordre $y^{\prime \prime}=w\left(x, y, y^{\prime}\right)$. Preisschriften gekrönt und hrsg. von der Fürstlich Jablonowskischen gesellschaft zu Leipzig. XXXII. Nr. XIII der mathematischenaturwissenschaftlichen section. S. Hirzel, Leipzig, 1896.

[Zeg96] Abdelghani Zeghib. Killing fields in compact Lorentz 3-manifolds. Journal of Differential Geometry, 43(4):859-894, 1996.

Martin Mion-Mouton, IRMA, 7 Rue René Descartes 67084 Strasbourg.

Email address: martin.mionmouton@math.unistra.fr 